Florida International University FIU Digital Commons

2016

\title{
Rate Kinetics and Molecular Dynamics of the Structural Transitions in Amyloidogenic Proteins
}

Timothy Michael Steckmann

tstec001@fiu.edu

DOI: $10.25148 /$ etd.FIDC001247

Follow this and additional works at: https://digitalcommons.fiu.edu/etd

Part of the Biological and Chemical Physics Commons

\section{Recommended Citation}

Steckmann, Timothy Michael, "Rate Kinetics and Molecular Dynamics of the Structural Transitions in Amyloidogenic Proteins" (2016). FIU Electronic Theses and Dissertations. 2978.

https://digitalcommons.fiu.edu/etd/2978

This work is brought to you for free and open access by the University Graduate School at FIU Digital Commons. It has been accepted for inclusion in FIU Electronic Theses and Dissertations by an authorized administrator of FIU Digital Commons. For more information, please contact dcc@fiu.edu. 


\title{
FLORIDA INTERNATIONAL UNIVERSITY \\ Miami, Florida
}

RATE KINETICS AND MOLECULAR DYNAMICS OF THE STRUCTURAL TRANSITIONS IN AMYLOIDOGENIC PROTEINS

\author{
A dissertation submitted in partial fulfillment of \\ the requirements for the degree of \\ DOCTOR OF PHILOSOPHY \\ in \\ PHYSICS \\ by
}

Timothy M. Steckmann 
To: Dean Michael R. Heithaus

College of Arts, Sciences, and Education

This dissertation, written by Timothy M. Steckmann, and entitled Rate Kinetics and Molecular Dynamics of the Structural Transitions in Amyloidogenic Proteins, having been approved in respect to style and intellectual content, is referred to you for judgement.

We have read this dissertation and recommend that it be approved.

Jin $\mathrm{He}$

$\overline{\text { Jaroslava Miksovska }}$

Prem P. Chapagain, Co-major Professor

Bernard S. Gerstman, Co-major Professor

Date of Defense: November 1, 2016

The dissertation of Timothy M. Steckmann is approved.

Dean Michael R. Heithaus

College of Arts, Sciences, and Education

Andrés G. Gil

Vice President for Research and Economic Development and Dean of the University Graduate School

Florida International University, 2016 


\section{DEDICATION}

I dedicate this dissertation to my beloved parents. 


\section{ACKNOWLEDGMENTS}

I would like to express great appreciation to my major professor Bernard Gerstman and co-major professor Prem Chapagain for their guidance and help during my entire research work and dissertation writing. An expression of warm gratitude goes to the secretaries of the physics department whose dedication provided the necessary administrative duties for my Ph.D. Thanks goes to the computer expert Barry Branch who provided the necessary computer support for my research group. I would also like to thank my dissertation committee members Professor Jin He and Professor Jaroslava Miksovska for their helpful suggestions and comments. I am thankful to former fellow graduate student Yuba for the molecular dynamics simulations I sampled to do my own molecular dynamics simulations. I am grateful to Zubair who was the undergraduate who worked with me on population dynamics of protein structures. Thanks to the Biophysics Research Group members Jeevan, Nisha, Rudra, Chandra, Choodamani, and Norma for their help and cooperation.

I would like to thank my parents who always supported and encouraged me toward excellence during the long journey of my education. 
ABSTRACT OF THE DISSERTATION

RATE KINETICS AND MOLECULAR DYNAMICS OF THE STRUCTURAL TRANSITIONS IN AMYLOIDOGENIC PROTEINS

by

Timothy M. Steckmann

Florida International University, 2016

Miami, Florida

Professor Bernard S. Gerstman, Co-major Professor

Professor Prem P. Chapagain, Co-major Professor

Amyloid fibril aggregation is associated with several horrific diseases such as

Alzheimer's, Creutzfeld-Jacob, diabetes, Parkinson's and others. The process of amyloid aggregation involves forming myriad different metastable intermediate aggregates. Amyloid fibrils are composed of proteins that originate in an innocuous $\alpha$ helix or random-coil structure. The $\alpha$-helices convert their structure to $\beta$-strands that aggregate into $\beta$-sheets, and then into protofibrils, and ultimately into fully formed amyloid fibrils. On the basis of experimental data, I have developed a mathematical model for the kinetics of the reaction pathways and determined rate parameters for peptide secondary structural conversion and aggregation during the entire fibrillogenesis process from random coil to fibrils, including the molecular species that accelerate the conversions. The specific steps of the model and the rate constants that are determined by fitting to experimental data provide insight on the molecular species involved in the fibril formation process. To better understand the molecular basis of the protein structural transitions and aggregation, I report on molecular dynamics (MD) 
computational studies on the formation of amyloid protofibrillar structures in the small model protein $\operatorname{cc} \beta$, which undergoes many of the structural transitions of the larger, naturally occurring amyloid forming proteins. Two different structural transition processes involving hydrogen bonds are observed for aggregation into fibrils: the breaking of intrachain hydrogen bonds to allow $\beta$-hairpin proteins to straighten, and the subsequent formation of interchain hydrogen bonds during aggregation into amyloid fibrils. For my MD simulations, I found that the temperature dependence of these two different structural transition processes results in the existence of a temperature window that the $\operatorname{cc} \beta$ protein experiences during the process of forming protofibrillar structures. Both the mathematical modeling of the kinetics and the MD simulations show that molecular structural heterogeneity is a major factor in the process. The MD simulations also show that intrachain and interchain hydrogen bonds breaking and forming is strongly correlated to the process of amyloid formation. 


\section{TABLE OF CONTENTS}

CHAPTER

PAGE

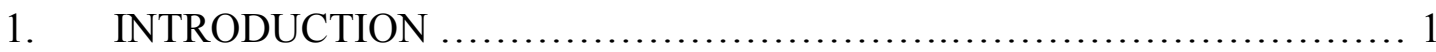

1.1 What is Already Known About the Fibrillization Process ................. 1

1.2 Free Energy ........................................................... 3

1.3 Hydrogen Bonds and Aggregation ................................... 5

1.4 Stages in the Fibrillization Process ................................... 5

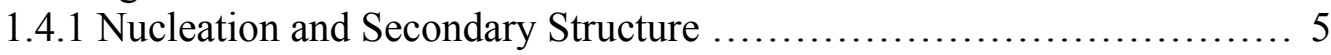

1.4.2 Steric Zipper ..................................................... 6

1.4.3 Hairping Straightening ........................................... 7

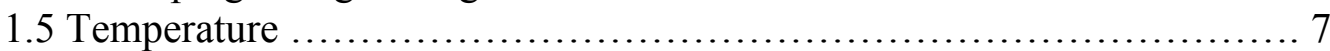

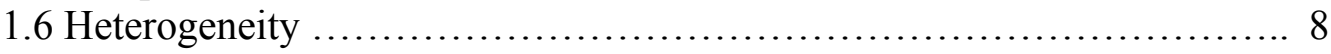

1.7 Work Performed on Amyloid-Like Fibrillization ......................... 9

1.7.1 Mathemtical Modeling of Real A $\beta$ Proteins to Gain Insight Into Various

Steps ................................................................ 9

1.7.2 Cc $\beta$ Molecular Dynamics Computer Simulations to Uncover Molecular

Level Details ....................................................... 11

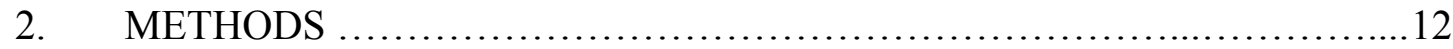

2.1 Mathematical Modeling of Oligomer Population Kinetics ................ 12

2.1.1 Introduction to Compartmental Modeling ............................... 13

2.1.2 Compartmenal Model Used In My Work ...............................19

2.1.3 Numerically Solving the Equations ................................ 20

2.2 Molecular Dynamics of Amyloid Formation ............................. 24

$2.2 .1 \mathrm{Cc} \beta$ Peptide .................................................... 24

2.2.2 Molecular Dynamics Computer Simulations .......................... 25

2.2.3 Details of Molecular Dynamics .................................... 26

2.2.3.1 Bonded Interactions ........................................... 26

2.2.3.2 Non-Bonded Interactions ..................................... 28

2.2.3.3 Non-Bonded Interaction Cut-Off Distances ...........................30

2.2.4 Numerical Time Integration for the Evolution of My System ............ 31

2.2.5 Replica Exchange Molecular Dynamics ................................ 35

2.2.6 Implicit Solvation ............................................... 36

2.2.7 Confining Potentials and Periodic Boundary Conditions ................. 38

2.3 My Particular Molecular Dynamics Simulation ........................ 38 
3. MOLECULAR RATE CONSTANTS FOR AMYLOID $\beta$

FIBRILLOGENESIS........................................................ 40

3.1 Description of $A \beta$ Proteins ...................................... 40

3.2 Description of Various Structures in My Model ........................... 41

3.3 Results ......................................................... 42

3.3.1 Model of Reaction Pathways and Experimental Data .................. 43

3.3.2 Modeling of the Lag Phase ........................................ 48

3.3.3 Modeling of the Rapid Drop-Off at Long Times in $\alpha$-Helix

Concentration ....................................................... 51

3.4 Numerical Results Using My Model to Fit the Experimental Data ........ 52

3.4.1 Co-Solvents ............................................................... 56

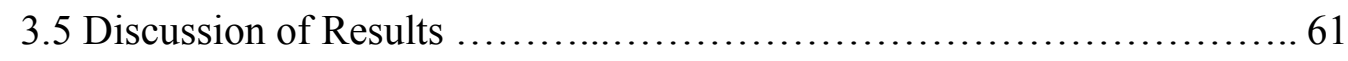

4. RESULTS OF MD COMPUTER SIMULATIONS FOR PROTOFIBRILLAR

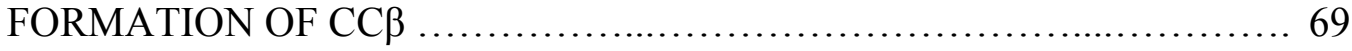

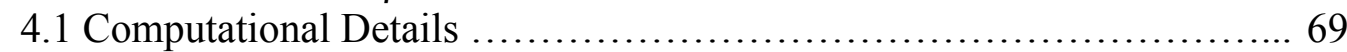

4.2 Description of $\beta$-Sheet (Nucleus) Formation ......................... 70

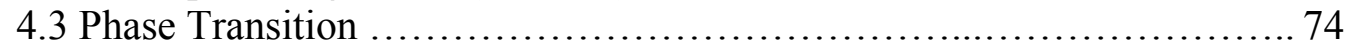

4.4 Validity of Results .................................................. 76

4.5 Temperature Window of $\beta$-Sheet Formation …......................... 80

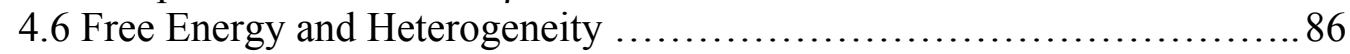

4.7 Protein to Protein Interactions ....................................... 88

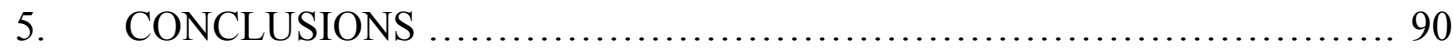

5.1 Hydrogen Bonds ................................................. 90

5.2 Heterogeneity ..................................................... 94

5.3 Directions for Future Work .......................................... 96

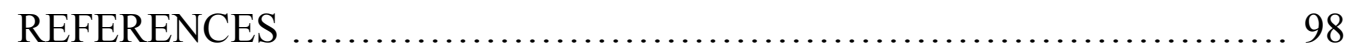

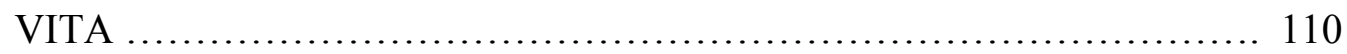




\section{LIST OF FIGURES}

FIGURES

PAGE

Figure 1.1: Structure schematics ........................................ 2

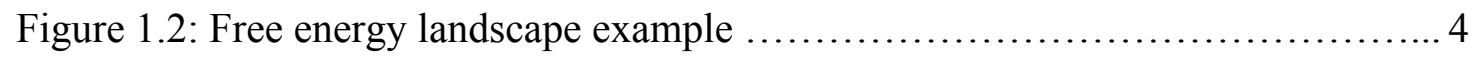

Figure 2.1: Radioactive decay schematic .................................. 13

Figure 2.2: Generalized schematic for transitions between two species .................14

Figure 2.3: Fictitious, homogeneous sub-compartment species inserted to mimic

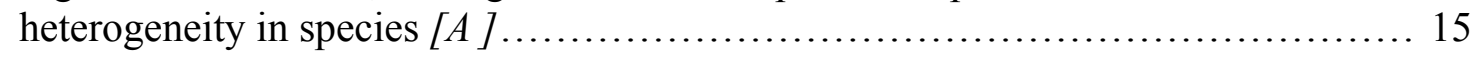

Figure 2.4: Schematic of sigmoidal growth of the population of species $[B] \ldots \ldots \ldots 16$

Figure 2.5: Initial, $\mathrm{t}=0$ populations for a system with one fictitious intermediate

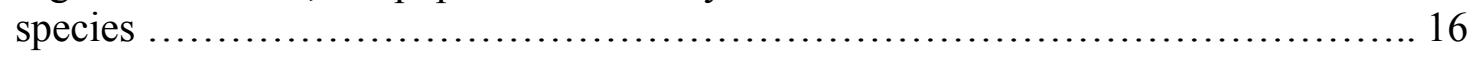

Figure 2.6: Populations for a system with one fictitious intermediate species (Fig. 2.4) after enough time has elapsed for significant transitions to occur

Figure 2.7: Populations for a system with one fictitious intermediate species (Fig. 2.4) at long times when most of the particles have completed the transitions to $B$

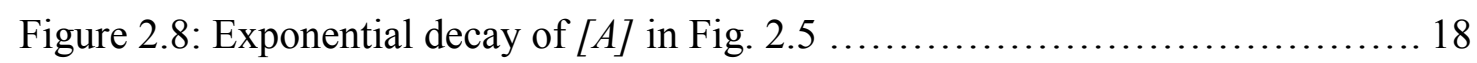

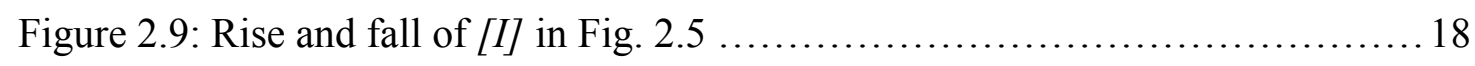

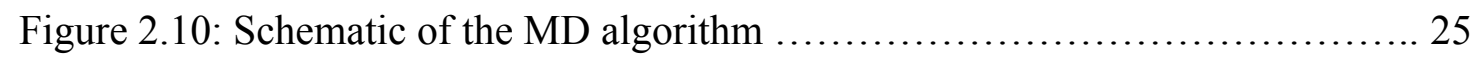

Figure 3.1: A state transition model of the structural conversion process of the A $\beta$

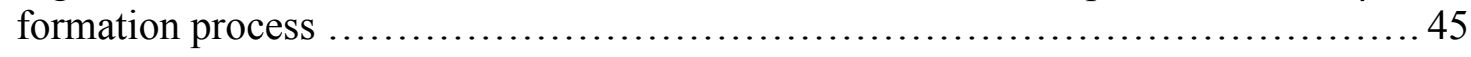

Figure 3.2: Experimental data (points) from Kirkitadze et al. fit with theoretical

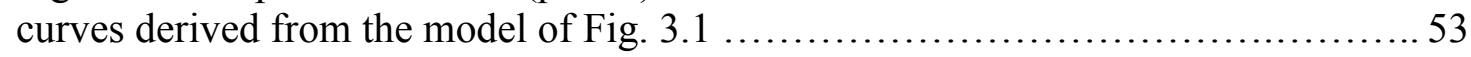

Figure 3.3: (a) The experimental data points from Walsh et al. showing the secondary structure content obtained by deconvolution of CD spectra. (b-d) Data points from Fezoui and Teplow showing the temporal changes in the $\beta$-structure content for $\mathrm{A} \beta(1-40)$ fibrillogenesis at different TFE concentrations 
Figure 3.4 Schematic energy landscape of $\mathrm{A} \beta$ secondary structure conversion as a function of arbitrary structural reaction coordinates $x$ and $y$

Figure 3.5: Theoretical fits (-------) to experimental data ( $\square$ ) for the number of monomers $n$ per aggregate for two different experiments. (a) Lomakin et al., (b) Kusumoto et al.

Figure 4.1: Frames from the REMD simulations showing steps in the helix structural transition to $\beta$-strands and the formation of $\beta$-sheets

Figure 4.2: The nematic order parameter $S$ for the proteins that make up the largest

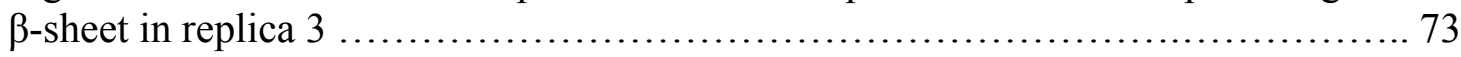

Figure 4.3: The heat capacity of my 12 chain system as a function of temperature $\ldots 75$

Figure 4.4: The number of chains in the largest aggregate as a function of temperature, averaged over the last $200 \mathrm{~ns}$

Figure 4.5: From replica three, positively charged (blue) and negatively charged (red) sidechains provide strong binding interactions: (a) between two $\beta$-sheets to hold the sheets together (b) on the outside of the two $\beta$-sheets to allow the addition of more $\beta$-sheets to the aggregate

Figure 4.6: Stability of the largest aggregate based upon a parameter $\chi_{\mathrm{n}}$ that measures the fluctuations in the number of chains in the largest aggregate

Figure 4.7: Number of interchain hydrogen bonds versus time. (a) $380 \mathrm{~K}$ (b) $472 \mathrm{~K}$ (c) $507 \mathrm{~K}$ (d) $600 \mathrm{~K}$

Figure 4.8: Average number of hydrogen bonds as a function of temperature 82

Figure 4.9: The average number of chains that are in a hairpin structure as a function of temperature 83

Figure 4.10 Free energy at $380 \mathrm{~K}$ for all $300 \mathrm{~ns}$ (minus $20 \mathrm{ps}$ from initialization and

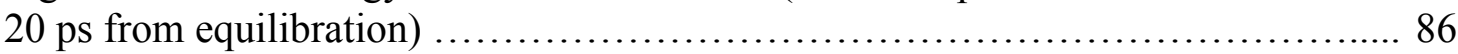

Figure 4.11 Free energy at $507 \mathrm{~K}$ as a function of $d_{e e}$ and $S$ for the last $200 \mathrm{~ns} \ldots \ldots 87$

Figure 4.12: Last frame of replicas three, 14, and 16 next to each other 88 


\section{ABBREVIATIONS AND ACRONYMS}

A $\beta \quad$ Amyloid beta

AD Alzheimer's Disease

APP Amyloid Peptide Precursor

BSE Bovine Spongiform Encephalopathy (mad cow disease)

CHARMM Chemistry at Harvard Molecular Mechanics

CTMD Constant Temperature Molecular Dynamics

EEF1 effective energy function-1

LJ Lennard-Jones

MD Molecular Dynamics

MMTSB Multiscale Modeling Tools for Structural Biology

NAMD Not Another Molecular Dynamics

NMR Nuclear Magnetic Resonance

PBC Periodic Boundary Condition

PDB Protein Data Bank

PDB ID Protein Data Bank Identification

$\mathrm{pH} \quad$ power (or potential) of Hydrogen

REMD Replica Exchange Molecular Dynamics

rk2 Runga Kutta second-order

rk4 Runga Kutta fourth-order

TFE Trifluoroethanol 


\section{INTRODUCTION}

Amyloid fibrils are protein structures that are associated with many debilitating human diseases such as Alzheimer's(AD), Parkinson's, Creutzfeldt-Jakob and diabetes, and in animal diseases such as BSE.

Amyloid fibrils are composed of proteins that originate in an innocuous $\alpha$-helix or random coil structure. The proteins convert their structure to $\beta$-strands that aggregate into $\beta$-sheets, and then into protofibrils, and ultimately into fully formed amyloid fibrils that are composed of about 600 protein chains. Although humans have evolved defenses against aggregation, ${ }^{1}$ unfortunately amyloid aggregation still occurs in humans. Pre-fibrillar $\beta$-aggregate structures have been found to be highly neurotoxic, and are more neurotoxic than mature amyloid fibrils or amyloid plaques. ${ }^{2,3,4}$ It remains uncertain which of the specific aggregates (protofibrils, protofilaments, or small oligomers) is most dangerous. ${ }^{5,6,7,8}$ A detailed molecular-level understanding of the formation process of amyloid fibrils is crucial for determining the molecular species that are toxic and for developing methods to slow down or prevent these horrific diseases.

Research on amyloid fibril formation overlaps strongly with prion related diseases. ${ }^{9}$ It also has potential nanoscience and material engineering applications since amyloid fibrils are especially strong mechanically. ${ }^{10,11}$

\subsection{What Is Already Known About the Fibrillization Process}

Amyloid fibrillation is a type of protein aggregation. Small $\beta$-aggregates form protofilaments which are composed of $\beta$-sheets parallel to each other. These 
protofilaments then wrap around each other like strands of rope to make protofibrils.

Protofibrils grow to form fibrils, which are insoluble. All fibrils have rope-like, twisted structures that reflect that they are assembled from filamentous structures. Fibrils can grow either by attaching of single monomers or attaching of $\beta$-aggregates. Figure 1.1 shows schematics of a single $\beta$-sheet, a protofilament, a protofibril and a fibril.

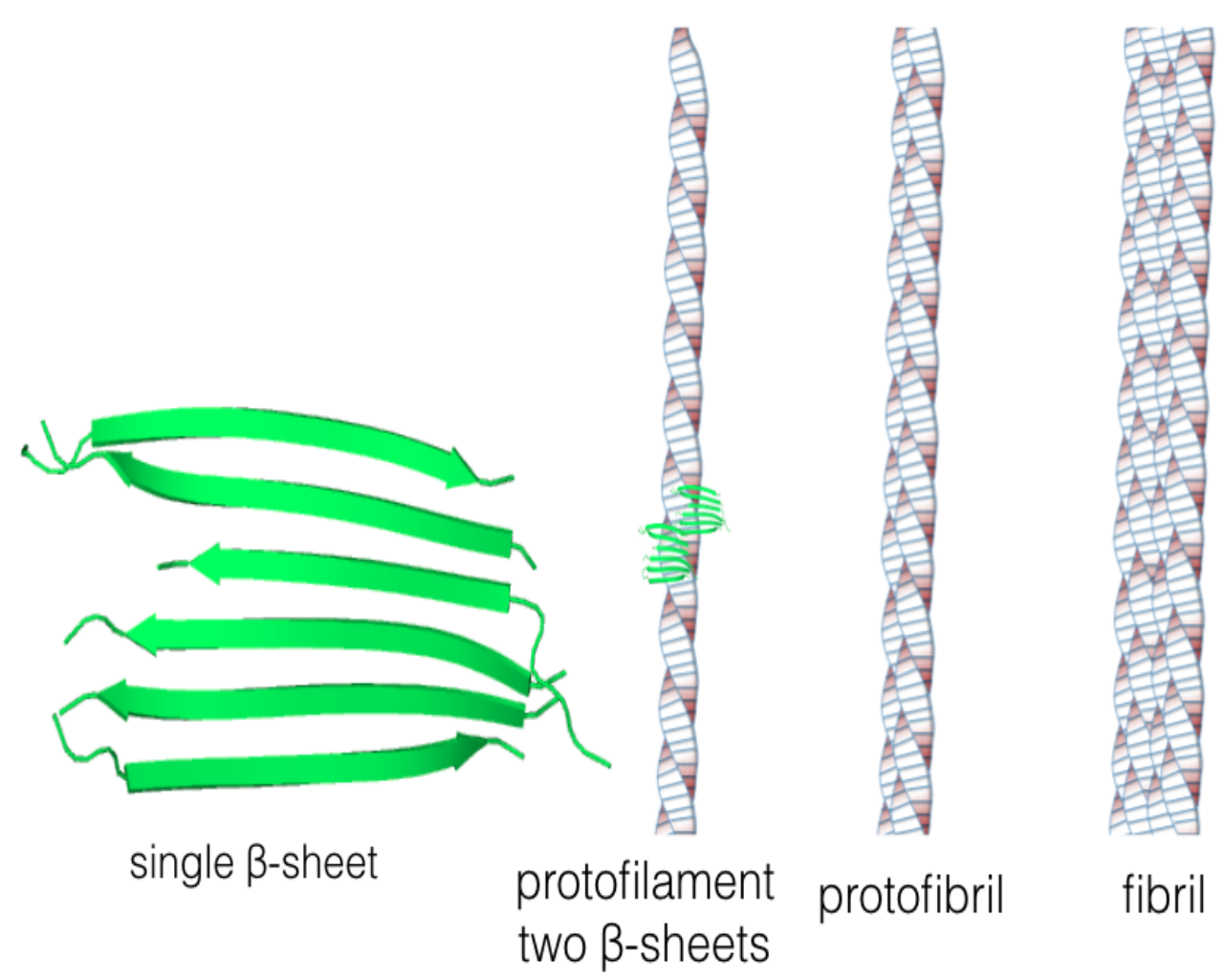

Fig. 1.1 Structure schematics. Schematic of a single $\beta$-sheet was done using Pymol. Pictures of a protofilament, a protofibril, and a fibril were done using PowerPoint following a diagram by David Talaga. ${ }^{12}$

Experimentalists have created amyloid fibrils in vitro. Solid state NMR measurements have shown the general structure of fibrils. However, detailed information about the kinetics of the fibrillization process is incomplete. 
Unfortunately, in vivo experiments are hard to perform and yield only the concentration of fibrils, but not the concentration of the precursors. In vitro studies allow measurements of the concentrations of some structural stages but the aggregation kinetics are unclear since the experimental techniques do not allow a distinction between individual intermediate $\beta$-structures. Likewise, detailed information about the molecular dynamics of various stages is also incomplete. Some information is available but details on a molecular level are experimentally difficult to obtain because it is hard to crystallize various $\beta$ precursor structures such as protofibrils.

Slight variations in experimental preparation procedures such as purification and synthesis may significantly change the peptide system's fibrillogenic properties. An additional complication is that a single system of proteins of the same species tends to have a heterogeneous combination of aggregates. One common observation is that increases in concentration of proteins tend to speed up aggregation. ${ }^{13,14,15}$

Several processes of protein aggregation often happen concomitantly with amyloid formation. Some of these processes create amorphous aggregates. ${ }^{16}$ Although amorphous aggregates are associated with misfolding diseases, some amorphous aggregates never form into amyloids. Some aggregates are small soluble oligomers, which can be swept out by the body. ${ }^{16}$

\subsection{Free Energy}

The idea for using free energy landscapes to describe different states in the fibrillation process came from protein folding. ${ }^{11}$ The free energy is a complicated landscape in amyloid formation since amyloid formation involves both protein folding 
and several proteins interacting with each other. ${ }^{11}$ However, useful information can still be obtained from free energy landscapes of amyloids and their precursors. An example of a free energy landscape to describe the aggregation process is given in Fig. 1.2. It is taken from Figure 8.2c of Amyloid Fibrils and Prefibrillar Aggregates. ${ }^{11}$ Amyloid fibrils in Fig. 1.2 clearly correspond to the lowest free energy minima.

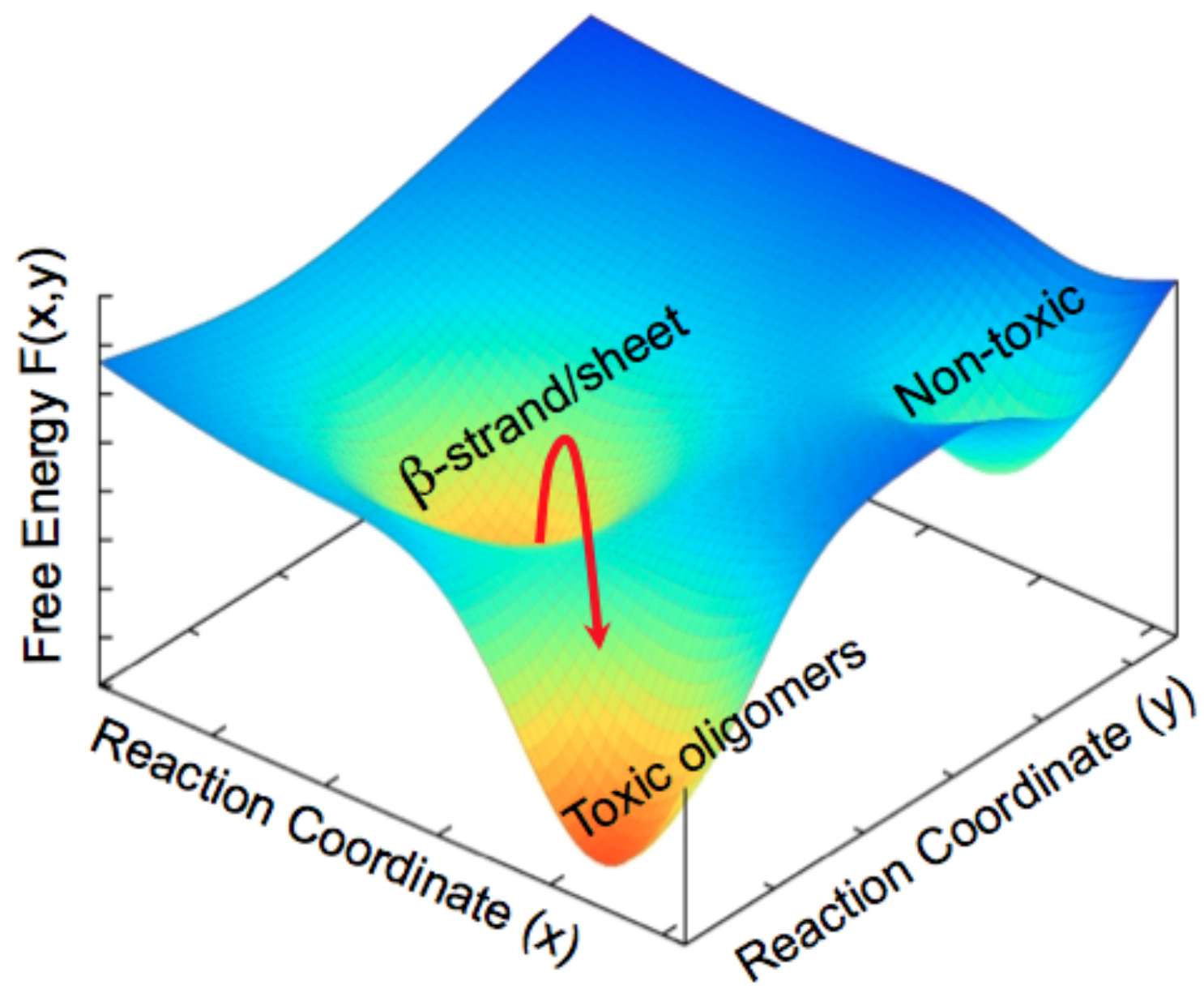

Fig. 1.2. Free energy landscape example. 


\subsection{Hydrogen Bonds and Aggregation}

Any protein can undergo amyloid aggregation, ${ }^{17}$ which may be because the formation and stabilization of $\beta$-sheets involves interchain hydrogen bonds between protein backbones ${ }^{17}$ which are common to all amino acids. Different hydrogen bonding patterns between backbones correspond to the different stages in the folding/aggregation process.

Simulation of two short $A \beta(16-22)$ peptides by Santini et al. ${ }^{18}$ showed multiple complex routes to fibrillization involving various networks of hydrogen bonds. Conformational properties of amyloids and their precursors can be partially described by the number of interchain and intrachain hydrogen bonds. There are many intrachain hydrogen bonds when a protein is in an $\alpha$-helical secondary structure or a hairpin conformation, whereas there are many interchain hydrogen bonds among proteins when they are in a $\beta$-sheet. Interchain hydrogen bonds form cooperatively with other interchain hydrogen bonds in the aggregation process.

\subsection{Stages in the Fibrillization Process}

\subsubsection{Nucleation and Secondary Structure}

According to classical nucleation theory, a nucleus is an aggregate of several proteins. It is the smallest aggregate size for which the rate of association is higher than the rate of disassociation. Once the nucleus is formed, the aggregation becomes a downhill polymerization process. What typically happens is, by chance, a group of proteins stick together with a number of proteins large enough that the energy loss is 
greater than the entropy loss due to another protein joining the aggregate. Although classical nucleation theory talks about a nucleus as being made up of several proteins, a nucleus can be as small as a single protein (as in the case of transthyretin). ${ }^{19}$ It could even be made up of a few residues of a protein.

The typical aggregation process involves some $\alpha$-helices or proteins that have some regions of $\alpha$-helix in them that eventually convert to $\beta$-strands as the aggregates undergo reorganization. In an article written by Lomakin et al. ${ }^{2}$ the proteins come together to form micelles, which are loosely-bound disordered aggregates. These micelles then break apart into nuclei, as described in my article ${ }^{20}$ and described later in Chapter 3. The initial assembly of proteins is like a micelle. The micelle or micelle-like aggregate breaks up into single proteins or small aggregates mostly in the $\beta$-strand state. $^{20}$ The process from single proteins to $\beta$-sheet is described as a "nucleated conformational conversion" by Cheon et al. ${ }^{21}$

\subsubsection{Steric Zipper}

The steric zipper conformation helps to stabilize amyloid fibrils. A steric zipper is a pair of parallel $\beta$-sheets. $\beta$ sheets come together due to the hydrophobic force and their side chains interlock. Their van der Waals forces hold them together. The steric zipper has no water molecules between the two $\beta$-sheets, causing it to be a dry

interface. ${ }^{22,23,24,11}$ Two different steric zippers can join through a wet interface. ${ }^{24} \mathrm{~A}$ wet interface has water and many hydrogen bonds between the side chains. The steric zipper is a common configuration of the protofilament. ${ }^{25}$ Some protofilaments contain only one steric zipper whereas some contain several. 


\subsubsection{Hairpin Straightening}

Hairpins are stabilized by intrachain hydrogen bonds and hydrophobicity. For general amyloid proteins, the intermediate aggregates are partly composed of $\beta$-strands in the hairpin state and/or ones that are extended. The oligomers tend to have a higher percentage of $\beta$-strands in the hairpin state than the fibrils. The book Molecular Modeling at the Atomic Scale by Zhou ${ }^{26}$ explains that the hairpin tends to serve as the growing end of the sheet. However, hairpins at the edges of oligomers tend to impede other proteins joining the aggregate. These hairpins needs to straighten out before bonding to other proteins. Zhou explains that hairpins can straighten out becaue of electrostatic forces. ${ }^{26}$ Many have suggested that key nucleation steps in the amyloid fibril formation process involve $\beta$-hairpin conformations.

\subsection{Temperature}

Increases in temperature have the effect of speeding up the aggregation process. ${ }^{27}$ Hairpins can also straighten out when exposed to high temperature and not interacting with other proteins. High temperature causes the protein not interacting with other proteins to be entropy-driven and remain highly flexible in random-coil configurations, rather than trying to lower its energy by intrachain hydrogen bonds. If the temperature is high enough, the aggregation process may not involve any $\alpha$-helices and instead just $\beta$-strands and random coils.

It has been suggested that most proteins have segments capable of forming amyloid fibrils if flexible enough, but most proteins have evolved to effectively conceal these segments. ${ }^{1}$ Although only certain proteins undergo aggregation in vivo, almost 
any globular protein can be made to form amyloid aggregates by denaturing it with heat or chemicals. ${ }^{1}$

\subsection{Heterogeneity}

It is hard to overstate the heterogeneity of amyloid precursors. Simulation of two short $A \beta(16-22)$ peptides by Santini et al. ${ }^{18}$ showed significant heterogeneity in forming a dimer. The heterogeneity of much larger amyloid forming systems is likely to have significantly more heterogeneity than the system of $A \beta(16-22)$ peptides by Santini et al. ${ }^{18}$ Amyloid precursors can potentially assume many conformations that are structurally distinct. ${ }^{11,20,28,29}$ No structure element common to all oligomers has been found. Some aggregates have parallel $\beta$-sheets and some have antiparallel $\beta$-sheets. ${ }^{30}$ Some aggregates have both parallel and antiparallel $\beta$-sheets. Oligomers tend to have different numbers of proteins.

Another cause of heterogeneity is that for an aggregating system, many of the

proteins may be undergoing secondary structure conversions. ${ }^{11}$ Although $\alpha$-helices are not a main constituent of the ordered fibril structure, there can be $\alpha$-helices in the intermediate aggregates. There is also a significant amount of heterogeneity in the fibrils. For instance, fibrils can be composed of both anti-parallel and parallel $\beta$-sheets. They also can have different $\beta$-sheet registries. ${ }^{31}$ 


\subsection{Work Performed on Amyloid-Like Fibrillization}

\subsubsection{Mathematical Modeling of Real A $\beta$ Proteins to Gain Insight into Various Steps}

An extensively studied, naturally occurring peptide system is the amyloid beta precursor $A \beta$ peptide, which is responsible for amyloid deposits in AD. Naturally occurring $A \beta$ peptides have a length in the range of 39-43 amino acids. Experiments have elucidated valuable structural information of variants such as $A \beta(10-35), A \beta(1-$ 40) and $A \beta$ (1-42) peptides. Fibrillar structures are formed when $A \beta$ acquires a $\beta$ secondary structure. Though the molecular mechanisms of fibrillation are still uncertain, it is known that $A \beta$ fibril formation is a multi-step nucleated polymerization process that involves soluble oligomeric intermediates called protofibrils. ${ }^{30}$

Many different theoretical models have been created for the rates of the

processes of aggregation. ${ }^{32,33,34,35}$ These models, however, are unable to explicitly deal with the fact that amyloid precursors are heterogeneous.

Kinetic models that include reaction pathways and rate parameters for the various stages of the process can be helpful toward understanding the dynamics on a molecular level. Rates for peptide structural transformations that nucleate $\beta$-structure, as well as rates for aggregation/polymerization into fibrils are especially interesting. There are experimental data on rate parameters for some of the stages of the fibril formation process. The stages that have experimental data can have protein specifics be accurately measured. These include the investigations on the elongation and nucleation 
rate parameters for fibrillogenesis at low $\mathrm{pH}$, rates of nuclei production and association, and lag time and rate of aggregation. Experiments have shed light on aspects of the process of $A \beta$ aggregation from random coil to fibril. Kirkitadze et al. ${ }^{28}$ were able to measure the temporal changes of concentrations of different types of secondary structures: random coil, $\alpha$-helix, $\beta$-strand/sheet, and $\beta$-turn for both $\mathrm{A} \beta(1-40)$ and $A \beta(1-42)$. These measurements were done over a period of 20 days for $A \beta(1-40)$ and 10 days for $A \beta(1-42)$, allowing enough time for fibrillogenesis to occur. Fezoui and Teplow $^{36}$ measured the concentration of prefibrillar and fibrillar $\beta$-structure as a function of time in different solvent conditions. Walsh et al. ${ }^{37}$ measured the concentration of various secondary structures as a function of time during the aggregation process.

On the basis of the experimental data, I have developed a mathematical model to determine viable reaction pathways and rate parameters for peptide secondary structural conversion and aggregation during the entire fibrillogenesis process from random coil to fibrils, including the species that accelerates the conversions. The numerical solutions yield graphs of concentrations of different molecular species versus time. With the proper choice of transition rate parameters, my model is able to nicely fit the time dependence of the concentrations measured experimentally by Kirkitadze et al., ${ }^{28}$ Walsh et al., ${ }^{37}$ and Fezoui and Teplow. ${ }^{36}$ The model and the rate constants include different molecular structural stages in the nucleation and polymerization process and provide insight on the molecular species involved in the fibril formation process.

Determination of rate parameters for the various peptide structural conversion steps during fibril formation may be helpful in developing efficacious therapies for AD. 


\subsubsection{Ce $\beta$ Molecular Dynamics Computer Simulations to Uncover Molecular Level Details}

Molecular dynamics (MD) computer simulations provide insight into the molecular details of various steps, such as the rearrangement of hydrogen bonds. I simulated a system with $12 \operatorname{cc} \beta$ proteins. ${ }^{38,39,40,41,42,43} \operatorname{cc} \beta$ is a de novo protein that is naturally in coiled-coil trimers at low temperatures but transitions to amyloid fibrils at high temperatures. I used Replica Exchange Molecular Dynamics (REMD) to model the aggregation process of $\operatorname{cc} \beta$. REMD makes it so that the system being simulated climbs over energy barriers in much shorter times than in constant temperature MD. The temperatures I used were in a range of $380-600 \mathrm{~K}$.

I observed $\operatorname{cc} \beta$ developing protofilaments with extended $\beta$-strands, corroborating what was found by Strodel et al. ${ }^{40}$ From these MD computational studies, I was able to investigate changes in the hydrogen bond arrangements and calculate thermodynamic properties that characterize the temperature dependence of the structural transitions. 


\section{METHODS}

The association of fibrillar $\mathrm{A} \beta$ with $\mathrm{AD}$ has stimulated interest in the kinetics and dynamics of $A \beta$ fibril formation. Fibrillar $A \beta$ is a fibril-like structure composed of many $\mathrm{A} \beta$ proteins. The $\mathrm{A} \beta$ proteins have 39 to 43 amino acids. They are produced by cleavage from a larger precursor protein, APP.

I used two different theoretical and computational approaches for studying fibrillization. In order to investigate the kinetics of the different oligomerization stages, I developed a system of coupled, non-linear differential equations describing the time evolution of the population of the different oligomer species. My model incorporated the heterogeneity of the oligomers. To study the molecular dynamics, I used a computational approach. It is very difficult to gain from experiments a detailed description of small oligomer formation, because of the structural heterogeneity and transient nature of the small oligomers. In order to investigate the molecular details of the fibrillation process, I used MD computer simulations.

\subsection{Mathematical Modeling of Oligomer Population Kinetics}

The important issues that must be incorporated into a mathematical model of the kinetics of the fibrillization process are the many different stages of oligomerization, as well as the heterogeneity within some of the stages. Compartmental modeling is an important concept for the development of a good model. 


\subsubsection{Introduction to Compartmental Modeling}

Many people are familiar with compartmental analysis from radioactive decay. The $R$ compartment is the number of radioactive atoms that have not yet decayed. The $D$ compartment is the number of atoms that have decayed. The system is homogenous, with each radioactive atom having an identical rate constant $k$ of decay. Figure 2.1 shows the radioactive decay scheme.

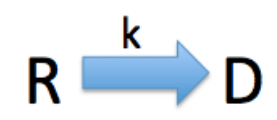

Fig. 2.1 Radioactive decay schematic.

The rate constant $k$ has the units of $1 /$ time $\left(\sec ^{-1}\right)$ and $1 / k \equiv \tau$ gives the characteristic time for decay of $R$. If the system is homogenous, the $D$ compartment follows a simple exponential with time, eq. (2.4). Assuming at time $\mathrm{t}=0,[R]=\left[R_{0}\right]$ and $[D]=0$

$$
\begin{gathered}
\frac{d[R]}{d t}=-k[R] \\
\rightarrow \frac{d\left[R^{\prime}\right]}{\left[R^{\prime}\right]}=-k d t^{\prime} \rightarrow \int_{\left[R_{0}\right]}^{[R]} \frac{d\left[R^{\prime}\right]}{\left[R^{\prime}\right]}=-k \int_{0}^{t} d t^{\prime} \rightarrow \ln \frac{[R]}{\left[R_{0}\right]}=-k t \\
\rightarrow[R]=\left[R_{0}\right] e^{-k t} \\
\frac{d[D]}{d t}=k[R]=k\left[R_{0}\right] e^{-k t} \\
\rightarrow \int_{\left[D^{\prime}\right]=0}^{[D]\left[D^{\prime}\right]}=k\left[R_{0}\right] \int_{t^{\prime}=0}^{t} e^{-k t^{\prime}} d t^{\prime} \rightarrow[D]=-\left[R_{0}\right]\left(e^{-k t}-1\right) \\
\rightarrow[D]=\left[R_{0}\right]\left(1-e^{-k t}\right)
\end{gathered}
$$


The starting equation (2.1) has a simple form because each radioactive atom had the same rate constant of decay, $k$. This led to a simple exponential function, equation (2.4) with respect to time for the growth of compartment $D$.

Now, let us consider a transition process in general, as shown in Figure 2.2.

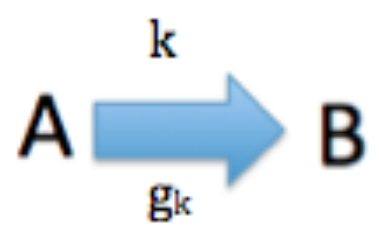

Fig. 2.2 Generalized schematic for transitions between two species. Species $A$ may have heterogeneity $g_{k}$ in the rate constant for transitions to $B$.

Using a simple equation like (2.1), $\frac{d[A]}{d t}=-k[A]$ would give a simple exponential for the population of compartment $B$, similar to equation (2.4) for radioactive decay. However, if each entity in $A$ has a different rate constant, equation (2.1) and the resulting equation (2.4) for the time-dependence of the population are no longer valid. The heterogeneity in $k$ can be expressed as

$$
\frac{d[A]}{d t}=-\int_{k} g_{k} k[A] d k
$$

where $g_{k}$ is the fraction of $A$ molecules with a specific value for the rate constant $k$, and the integral is over all possible values of $k$. If we substitute equation (2.5) for (2.1) above, the derivation leading to (2.4) becomes much more complicated than if we had used (2.1). Analytical solutions are not possible except for very special probability distributions of $g_{k}$. Especially important for the present work and for most systems, the distribution function $g_{k}$ of the molecular components is usually not known, and therefore it is not possible to include $g_{k}$ in the analysis. Fortunately, there is a way to 
create a mathematical model that allows the investigation of the kinetics of the different species that are known to transition amongst each other.

Mathematical modeling of heterogeneous kinetics can be performed without knowledge of $g_{k}$ if we substitute for the heterogeneity $g_{k}$ in the rate constant of one step by inserting several fictitious steps using fictitious sub-compartments. The population of each fictitious sub-compartment is treated as being homogeneous and therefore each transition from one compartment to the next can be described by a single rate constant, as shown in Figure 2.3.

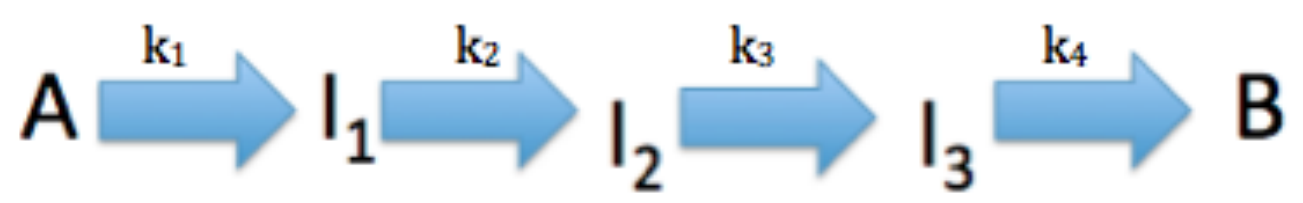

Fig. 2.3 Fictitious, homogeneous sub-compartment species inserted to mimic heterogeneity in species $[A]$.

The entities in $[A]$ are not really transforming into those intermediate compartments $\left[I_{1}\right],\left[I_{2}\right],\left[I_{3}\right]$ before arriving in $B$. However, the ansatz in the mathematical modeling that the entities in $[A]$ go through those fictitious intermediate compartments leads to sigmoidal time kinetics for $[B]$. This type of sigmoidal curve for [B] mimics the dynamics of the real system that has a significant amount of rate constant heterogeneity in species $[A]$. 


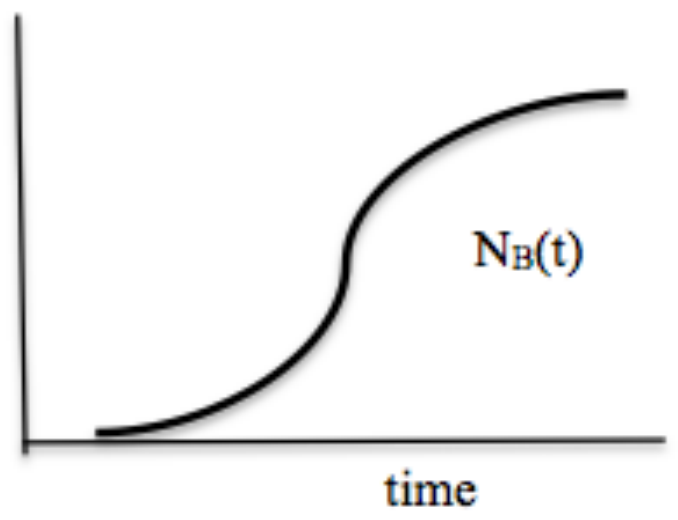

Fig. 2.4 Schematic of sigmoidal growth of the population of species [B].

To illustrate why these fictitious intermediate compartments produce a sigmoidal shape, I will use a simple case, with only one sub-compartment. Let us suppose that at $\mathrm{t}=0,100 \%$ of the entities are in compartment $A$, as shown in Fig. 2.5.

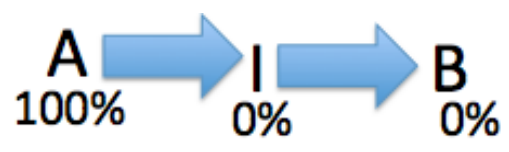

Fig. 2.5 Initial, $\mathrm{t}=0$ populations for a system with one fictitious intermediate species.

Entities in $A$ need to go through the intermediate compartment $I$ before the process of conversion from $[I] \rightarrow[B]$ commences. Because there is initially no population in $[I]$, the process of conversion to $[B]$ starts off slowly, with a lag time before the population of $[B]$ starts to increase.

Now, look at the situation at some intermediate time, after many transformations from $[A]$ to $[I]$ and transformations from $[I]$ to $[B]$ have occurred, in Figure 2.6. 


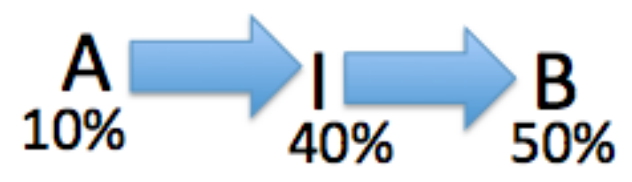

Fig. 2.6 Populations for a system with one fictitious intermediate species (Fig. 2.4) after enough time has elapsed for significant transitions to occur.

Now that there is significant population in $[I]$, the process of transformation to $[B]$ is much quicker.

Next, look at the situation at long times, when most of the entities are in compartment $[B]$ :

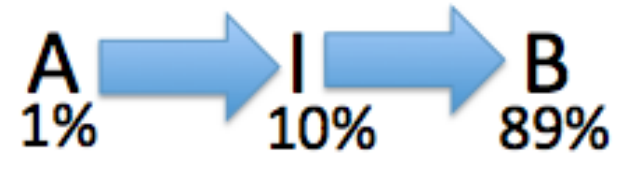

Fig. 2.7 Populations for a system with one fictitious intermediate species (Fig. 2.4) at long times when most of the particles have completed the transitions to $B$.

At long times, there are not many entities in [I] left to transform to $[B]$. The population of $[B]$ slows in growth rate as the system approaches its asymptotic population distribution. The greater number of intermediate compartments between $[A]$ and $[B]$, the more sigmoidal and less exponential $[B]$ will be.

So far, I have described only the behavior of $[B]$ from Fig. 2.2, and how Fig. 2.3 can be used to get numerical results for $[B(t)]$ when $g_{k}$ in Fig. 2.2 is not known. Since experimental measurements may be available for other species, e.g. [A], it is worth looking at their time development. In Figures 2.2, 2.3, and 2.5, the reactions occur only from left to right. This means that eventually, $[A]$ will disappear and [I] will disappear, but with different time profiles. The species $[A]$ experiences a simple first order 
reaction that depends only on $k_{l}$, and therefore $[A]$ decays exponentially, Fig. 2.8. In Fig. 2.5 with a single intermediate species, fictitous or real, the initial population of [I] is zero. It then rises (Fig. 2.9) by gaining population from [A] with a rate constant $k_{l}$, and then falls by converting to $[B]$ with a rate constant $k_{2}$. The relative values of $k_{l}$ and $k_{2}$ determine the details of the shape of the time dependence of [I], which is unlikely to be symmetric.

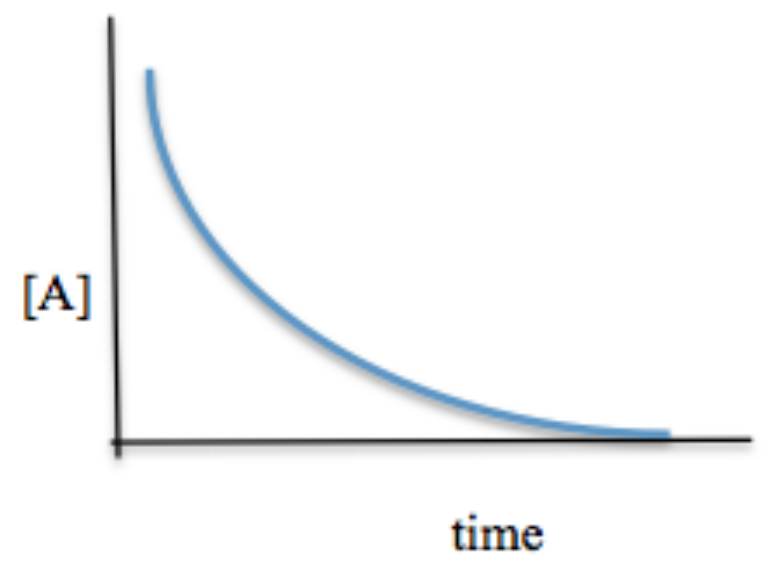

Fig. 2.8 Exponential decay of $[A]$ in Fig. 2.5.

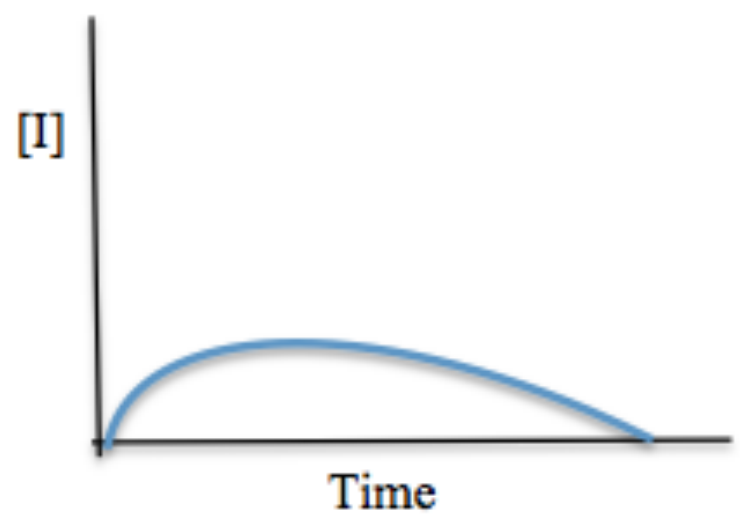

Fig. 2.9 Rise and fall of [I] in Fig. 2.5. 
The model that I described above and showed schematically in Fig. 2.3 through Fig. 2.9 is overly simple. The direction of transitions is only from left to right. Therefore, at long enough times, the only species that remains is the last one. Real systems are usually more complicated. Reactions can go in both directions, and there is also the possibility for alternate paths that skip steps, such as going directly from $A$ to $B$. Also, there may be back reactions that provide feedback, such as from $[B] \rightarrow[A]$. All of these possibilities must be taken into account to produce a realistic mathematical model of the kinetics that is more complicated than the simple example given above.

\subsubsection{Compartmental Model Used In My Work}

The use of differential equations for studying the change in protein compartments with time is described in Chapter 4 of Protein Folding Kinetics by Nolting. ${ }^{44}$ Nolting shows coupled differential equations that are simple enough to have analytic solutions. The differential equations in Protein Folding Kinetics did not explictily take into consideration the heterogeneity in the rate constants.

I used differential equations to study the changes in population in the following secondary structure configurations (species in the examples given above): random coil/ $\beta$-turn, $\alpha$-helix, $\beta$-strand/sheet. Each compartment was the percentage of amino acids from all the proteins in the system with a characteristic secondary structure. Many of the compartments also had structural characteristics in addition to the secondary structure.

On the basis of experimental data, I developed a mathematical model to determine viable reaction pathways and rate parameters for peptide secondary structural 
conversion and aggregation during the entire fibrillogenesis process from random coil to mature fibrils, including the species that accelerates the conversions. The numerical solutions yield graphs of concentrations of different molecular species versus time. The model and the rate constants include different molecular structural stages in the nucleation and polymerization process and provide insight on the molecular species involved in the fibril formation process. Determination of rate parameters for the various peptide structural conversion steps during fibril formation may be helpful in developing efficacious therapies for AD.

\subsubsection{Numerically Solving the Equations}

Finding an analytical solution to the complicated set of differential equations that I developed (see Chapter 3) would be extremely difficult, if not impossible. The number of equations that are coupled with each other is large, plus many of them are non-linear. Instead, I solved them numerically.

There are different numerical algorithms for solving differential equations. An overview of some of these techniques is given in the book

Computational physics by Newman. ${ }^{45}$ The numerical algorithms involve iterations over time steps.

A simple algorithm is Euler's Rule. The formula for Euler's rule with one dependent variable is:

$$
x(t+h)=x(t)+h \cdot f(x, t)
$$


with $d x / d t=f(x, t)$ and $\Delta t=h$ the size of the time step. The formula for $f(x, t)$ is assumed known, so that its value can be determined at each step. Iteratively using this equation yields a solution with an error on the order of $h^{2}$. The second order dependence is good because $h$ is a small quantity. There is only one equation to use for Euler's method and it's a simple equation that comes from a Taylor expansion. However, by making things more complicated, we can reduce the algorithmic error to a higher power of $h$.

The second-order Runge Kutta method uses the slope at the midpoint between points at $t$ and $t+h, t+\frac{1}{2} h$. The second-order Runge Kutta method requires a knowledge of $x\left(t+\frac{1}{2} h\right)$, which can be approximated using Euler's method: $x\left(t+\frac{1}{2} h\right)=x(t)+\frac{1}{2} h f(x, t)$. The equations used for second-order Runga Kutta ( $\mathrm{rk} 2)$ for a single dependent variable are:

$$
\begin{aligned}
& k_{1}=h f(x, t) \\
& k_{2}=h f\left(x+\frac{1}{2} k_{1}, t+\frac{1}{2} h\right) \\
& x(t+h)=x(t)+k_{2}
\end{aligned}
$$

The formula for $f(x, t)$ is assumed known, so that its value can be determined at each step. You use the first equation to get $k_{1}$. You then use the second equation to calculate $k_{2}$. Based on knowledge of $k_{2}$, you get the value of $x(t+h)$. The error for rk2 is of the order of $h^{3}$. 
Can we do better than rk2? How about fourth order Runga Kutta (rk4)? By adding linear combinations of Taylor expansions around various points, we can cancel terms in $h^{3}, h^{4}$ and so on. The equations for rk4 for a single dependent variable are:

$$
\begin{gathered}
k_{1}=h f(x, t) \\
k_{2}=h f\left(x+\frac{1}{2} k_{1}, t+\frac{1}{2} h\right) \\
k_{3}=h f\left(x+\frac{1}{2} k_{2}, t+\frac{1}{2} h\right) \\
k_{4}=h f\left(x+k_{3}, t+h\right) \\
x(t+h)=x(t)+\frac{1}{6}\left(k_{1}+2 k_{2}+2 k_{3}+k_{4}\right)
\end{gathered}
$$

For each iteration, you start with the first equation and do the rest of the equations the order in which they are written above. There are two more equations in rk4 than in rk2, which requires additional computational time, but the benefit is that it has an algorithmic error on the order of $h^{5}$, which means larger values of $h \equiv \Delta t$ can be used. With larger values of $h$, the overall number of time steps can be reduced. Many people feel rk4 offers the best balance between number of calculations needed for each time-step and algorithm accuracy. It is by far the most commonly used method. For a system of coupled differential equations with more than one dependent variable, like my system, the equations are iteratively integrated for each dependent variable in parallel with the other dependent variables. 
Although in general rk4 is the best method for solving differential equations, it depends on the particular situation. Remember that algorithmic errors are not the only errors. There are also round-off errors. Round-off errors come from the fact that a computer does not use an infinite number of digits after the decimal point. Round-off errors may seem trivial, but they can accumulate to produce large errors. ${ }^{46}$ Round-off errors are especially prevalent in the above-mentioned techniques because they involve getting slopes numerically, which involves subtracting two numbers that are close to each other. Subtracting two numbers that are close to each other can produce serious round-off errors. ${ }^{46}$ The idea is to have a good balance between algorithmic accuracy and the number of steps in the calculations. This is accomplished through the proper selection of the algorithm and the size of the time step. There is even the idea of varying the step size while doing the rk4 integration. ${ }^{46}$

The differential equations representing the reaction pathway for my model were solved numerically using the computer software package Mathematica 7.0. Mathematica analyzes the differential equations and then determines which algorithm to use. Mathematica sometimes even switches after already starting to solve the equations if a better choice is found. 


\subsection{Molecular Dynamics of Amyloid Formation}

\subsection{1 ccß Peptide}

In order to perform detailed MD computational investigations, I focused my attention on a small, engineered peptide, $\operatorname{cc} \beta$ (PDB ID code 1S9Z). ${ }^{38,39,40,41,42,43} \operatorname{cc} \beta$, which has amino acid sequence SIRELEARIRELELRIG, responds to temperature changes by undergoing many of the structural transitions that occur in the amyloid formation processes of the larger, naturally occurring amyloid forming proteins and it has been demonstrated experimentally that coiled-coil trimers of $\operatorname{cc} \beta$ can transform their structure and form amyloid-like protofibrils. ${ }^{42}$ Experimental observations ${ }^{42}$ find that at a temperature of $277 \mathrm{~K}, \operatorname{cc} \beta$ exists as a stable coiled-coil $\alpha$-helix trimer, whereas at $310 \mathrm{~K}$ the helical structure converts to $\beta$-strands. MD investigations on a single trimer by Strodel et al. ${ }^{40}$ found that a $\beta$-sheet composed of three $\operatorname{cc} \beta$ chains is stable up to a temperature of $440 \mathrm{~K}$.

There are two important advantages to using $\operatorname{cc} \beta$ in investigations of amyloid formation: its small size, and the fact that the control parameter for inducing structural transitions is temperature. Strodel et al. ${ }^{40}$ performed MD simulations of a trimer of $\operatorname{cc} \beta$. In order to speed up the process, they used replica exchange MD (REMD) simulations with the EEF1 force field. They found many pathways for the transforming of the coiled-coil $\operatorname{cc} \beta$ trimer into a trimer $\beta$-sheet. They also found that only parts of the $\operatorname{cc} \beta$ peptides first convert to $\beta$ strands, which then further catalyzes the $\alpha$ to $\beta$ secondary structure conversion. ${ }^{40}$ 


\subsubsection{Molecular Dynamics Computer Simulations}

Molecular dynamics is a computational technique that simulates the dynamics of a system of atoms as a function of time. On the most fundamental level, the evolution of the system is described by quantum mechanics. However, using quantum mechanics would be computationally very expensive. Instead, the dynamics of the atoms are handled classically. Although the energy functions that are used are classical, the parameters (such as the spring constants representing bonds) come from experiments or from results of ab initio quantum mechanics calculations involving electronic orbitals. The use of classical dynamics with quantum mechanically determined parameters makes it so that the calculations are quick enough to model many-atom systems such as proteins, but accurate enough to produce simulations that reasonably agree with experiment. Figure 2.10 is a schematic of the basics of the MD algorithm.

\section{Basic MD Algorithm}

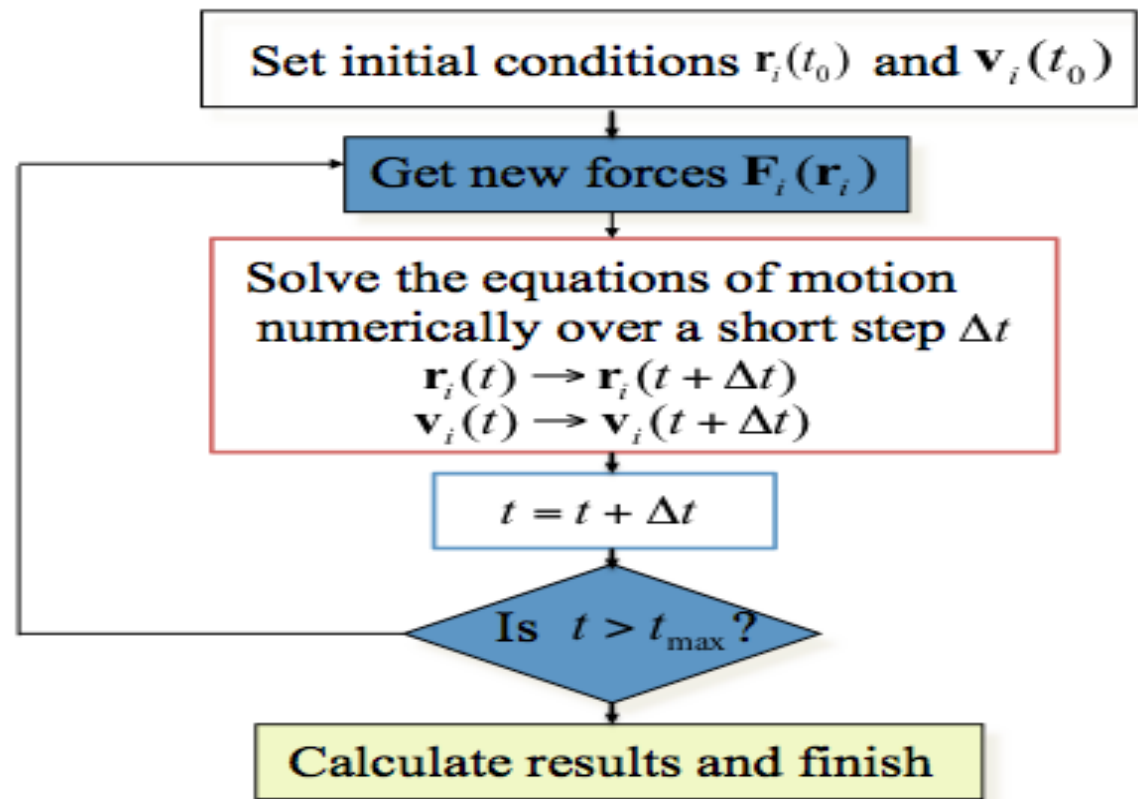

Figure 2.10 Schematic of the MD algorithm. 
There are different packages that perform MD, such as NAMD, Amber, and

CHARMM. I used CHARMM (Chemistry at Harvard Molecular Mechanics) for my

MD simulations. CHARMM is a versatile MD simulations package.

\subsubsection{Details of Molecular Dynamics}

The MD calculations compute the forces and energies based on the positions of all the atoms in the system. The interactions among atoms are classified into bonded and non-bonded.

\subsubsection{Bonded Interactions}

\section{Bond Stretching}

A chemical bond is modeled as a spring with a classical form of the potential energy (coming from Hooke's Law), but with the value of the bond spring constant $k_{b}$ derived from experiments or $a b$ initio quantum mechanics calculations:

$$
U(r)=\frac{k_{b}}{2}\left(r-r_{0}\right)^{2}
$$

with $r_{0}$ the equilibrium bond length. 


\section{Angle Bending}

The angle bending energy is the energy needed to bend a bond from its equilibrium value, $\theta_{0}$ :

$$
U(\theta)=\frac{k_{\theta}}{2}\left(\theta-\theta_{0}\right)^{2}
$$

in which $k_{\theta}$ is the force constant whose value is derived from experiment or $a b$ initio quantum calculations. The form of the energy is that of a simple harmonic oscillator, which is valid for small $\theta$.

\section{Torsions}

If four atoms $\mathrm{i}, \mathrm{j}, \mathrm{k}$, and $\mathrm{l}$ form a bonded quartet ( $\mathrm{i}$ bonded to $\mathrm{j}, \mathrm{j}$ bonded to $\mathrm{k}$, and $\mathrm{k}$ bonded to 1), a dihedral angle can be defined. The dihedral angle for a sequence of atoms $\mathrm{i}-\mathrm{j}-\mathrm{k}-\mathrm{l}$ is the angle between the normal to the plane defined by the atoms $\mathrm{i}-\mathrm{j}-\mathrm{k}$ and the normal to the plane defined by the atoms $\mathrm{j}-\mathrm{k}-1 .{ }^{47}$ The terms dihedral angle and torsion angle are often used interchangeably. The energy for the torsion angle $\varphi$ is:

$$
U(\varphi)=\sum_{n=1}^{N} \frac{k_{\varphi}}{2}[1+\cos (n \varphi-\delta)]
$$

In the above equation, $k_{\varphi}$ is a force constant (derived from $a b$ initio quantum calculations or experiments). $\delta$ is the phase factor that puts the minimum potential energy at the correct torsion angles. 
The idea is that $n$ is the periodicity of the dihedral angle, or the number of cycles for a full $360^{0}$ rotation. $^{48}$ For instance, $N=2$ means there is one potential energy function which has one minima and another energy function which has two minima, each $180^{\circ}$ apart. $N$ is usually only one, although it can go up to six. ${ }^{49}$

Improper torsions are used, when needed, to enforce the plane structure of a molecule. If instead of atoms $\mathrm{i}, \mathrm{j}, \mathrm{k}, \mathrm{l}$ bonded in a sequence, you have a starlike conformation with $\mathrm{k}$ bonded to atoms $\mathrm{i}, \mathrm{j}, 1$ you use an improper torsion angle. Atom $\mathrm{k}$ is called a branching point. Improper torsion energy can be used to obtain a particular geometry. The energy of an improper torsion angle is:

$$
U(\omega)=\frac{1}{2} k_{\omega}\left(\omega-\omega_{0}\right)^{2}
$$

with $\omega$ being the angle between the normal to the plane $\mathrm{i}, \mathrm{j}, \mathrm{k}$ and the normal to the plane $\mathrm{j}, \mathrm{k}, \mathrm{l}$.

\subsubsection{Non-Bonded Interactions}

Most of the non-bonded interactions are the electrostatic and van der Waals interactions. Non-bonded interactions are neglected between covalentlybonded atom pairs and atom pairs that are separated by only two covalent bonds ${ }^{49}$ because the covalent bonds incorporate the non-bonded interactions. 


\section{Electrostatic Interactions}

These interactions are the attractive and repulsive forces between charged atoms and molecules. The electrostatic energy function comes from Coulomb's law:

$$
U(r)=\sum_{i=1}^{N_{A}} \sum_{j=1}^{N_{B}} \frac{q_{i} q_{j}}{4 \pi \varepsilon r_{i j}}
$$

In the equation above, $N_{A}$ is the number of point charges in molecule $A$ and $N_{B}$ is the number of point charges in molecule $B$.

There are also electrostatic interactions within a molecule:

$$
U(r)=\sum \frac{q_{i} q_{j}}{4 \pi \varepsilon r_{i j}}
$$

where the sum is over all pairs of atoms in the molecule besides nearest and next-nearest pairs. The permanent partial atomic charges that are assigned at the beginning of an MD simulation are determined by making an initial guess and then performing calculations in an attempt to get the results of quantum mechanics calculations or experiments. The assigned partial charges are modified based on the discrepancy between the result of the initial guess and results from experiments or quantum mechanics calculations. The process is repeated until there is good agreement between assigned charges and experiments or quantum mechanics calculations. 


\section{Lennard-Jones Interactions}

The van der Waals interaction, which is the total energy of interaction between two non-bonded atoms, is approximated by the Lennard-Jones (LJ) $\operatorname{potential}^{50}$ :

$$
U(r)=E_{0}\left[\left(\frac{r_{0}}{r}\right)^{12}-2\left(\frac{r_{0}}{r}\right)^{6}\right]
$$

$E_{0}$ is the depth at the minimum in energy and $r_{0}$ is the separation of the atoms at the minimum. The first term gives the repulsion that comes from the Pauli Exclusion Principle when the electron clouds overlap. The last term gives the attraction that comes from induced dipole moments. The LJ energy makes it so that two atoms' nuclei stay a reasonable distance (but not too far) from each other. The reason eq. (2.21) is approximate is that it assumes atoms are spherical, since the energy function is independent of direction.

\subsubsection{Non-Bonded Interaction Cut-Off Distances}

Calculating every non-bonded interaction would not be feasible computationally for protein systems, since the number of non-bonded interactions scales as the square of the number of atoms and proteins have thousands or more atoms. A common way to reduce the number of calculations for the non-bonded interactions is to use a cut-off distance. 
A cutoff distance is chosen by the MD user, beyond which no interaction is calculated. Care must be used when deciding where to have the non-bonded interactions cut-off, since electrostatic energies have infinite range. This cutoff creates a discontinuity if the energy abruptly drops to zero, which can lead to unreliable results or program termination. A switching function is used to avoid any discontinuities in energy.

Three parameters are associated with the cutoff distance: ctonnb, ctofnb, and ctnb. The switching function affects the non-bonded energies beginning at ctonnb, the smallest of the three parameters. The switching function takes the interaction smoothly to zero at ctofnb. The distance for generating the nonbonded pair list is given by the value of ctnb. The non-bonded pair list is updated periodically, and saves computational time by not having to check all of the atoms at every step to see if they are within ctofnb. The value of ctnb is slightly larger than ctofnb. For my system, cuton was $16 \AA$, ctofnb was $18 \AA$, and cutnb was $20 \AA$.

\subsubsection{Numerical Time Integration for the Evolution of My System}

Molecular dynamics makes use of $\Sigma \boldsymbol{F}=m \boldsymbol{a}$ and $\boldsymbol{F}=-\boldsymbol{\nabla} U$ to calculate the acceleration of each atom based on the bonded and non-bonded forces of the surrounding atoms for each MD time-step. The acceleration is used to update the position and velocity of each atom for the next MD time-step. A time 
interval of $\sim 1 \mathrm{fs}$ is required to capture the highest frequency of motions of biomolecular systems, which is the vibrational motion of the chemical bond between a heavy atom and hydrogen. However, if the SHAKE command is used to constrain the bond length between a hydrogen and heavy atom a time-step of $\sim 2 \mathrm{fs}$ can be employed. SHAKE can be appllied to all the bonds and angles if selected. It uses an iterative scheme to exactly fix bond lengths. ${ }^{51}$ In the case of constraining the bond length between hydrogen and heavy atoms, SHAKE is an iterative procedure to adjust the hydrogen atoms after each step that the heavy atoms are integrated. ${ }^{48}$

Some techniques that numerically update the position and velocity of each atom given the acceleration are described below. These techniques are referred to as finite-difference methods. These numerical integration techniques have much in common with the numerical techniques used in the mathematical modeling of the kinetics that is described above.

\section{Taylor Expansion}

The simple Taylor expansion technique to advance the position and velocity with time of atom $n$ uses these two equations ${ }^{48}$ :

$$
\begin{gathered}
r_{n+1}=r_{n}+v_{n} \Delta t+\frac{1}{2} \frac{F_{n}}{m}(\Delta t)^{2} \\
v_{n+1}=\frac{r_{n+1}-r_{n}}{2 \Delta t}
\end{gathered}
$$


with $\Delta t$ as the time-step and $F_{n}$ represents the sum of the forces on atom $n$. This formulation is quite simple, but results in large errors.

\section{Verlet Integrator}

The Verlet Integrator is an improvement over the simple Taylor expansion. It is derived from using a backward Taylor Expansion and a forward Taylor expansion. ${ }^{48}$ The Verlet integrator uses this equation for advancing the position with time

$$
r_{n+1}=2 r_{n}-r_{n-1}+\frac{F_{n}}{m}(\Delta t)^{2}
$$

The velocity at each point can be estimated by:

$$
v_{n}=\frac{r_{n+1}-r_{n-1}}{2 \Delta t}
$$

The Verlet integrator gives high-precision values of position (errors on the order of $\Delta t^{4}$ ) and is independent of velocity propagation. ${ }^{48}$ However, the velocity propagation from the Verlet integrator can have large errors on the $\operatorname{order}^{48}$ of $\Delta t^{2}$ and the velocity can only be computed after the position is computed. It also requires a value of the position for the $n-1$ time step. Sometimes, a simple Taylor expansion is used to get the position at a fictitious 1 time step to get the algorithm started from the initial structure. (The starting structure is at time step zero.) 


\section{Leap-Frog Integrator}

The Leap-Frog integrator (also called Verlet Leap-Frog integrator) is a modification to the basic Verlet integrator. ${ }^{48}$ The Leap-Frog integrator uses these two equations for advancing the system with time:

$$
\begin{aligned}
& r_{n+1}=r_{n}+v_{n+1 / 2} \Delta t \\
& v_{n+1 / 2}=v_{n-1 / 2}+\frac{F_{n}}{m} \Delta t
\end{aligned}
$$

As can be seen from equations (2.26) and (2.27), the Leap-Frog Integrator is so called because during a time step $\Delta t$ the velocty leaps $1 / 2$ time step ahead of the position as it is calculated. When the position is calculated, it leaps $1 / 2$ time step ahead of the velocity. The current velocity can be calculated using:

$$
v_{n}=\left(v_{n+1 / 2}+v_{n-1 / 2}\right) / 2
$$

or other methods that introduce smaller errors than equation (2.28). The position in the Leap-Frog algorithm has an error for position of $\Delta t^{4}$, the same as Verlet. However, the error in the velocity calculated using Leap-Frog at half time steps is $\Delta t^{3}$, which improves over the Verlet algorithm of $\Delta t^{2}$. 
Simply decreasing $\Delta t$ to try to increase the precision would require more time steps to be calculated. This not only increases the computer time needed, but also increases the total round-off error. ${ }^{46} \mathrm{~A}$ rule of thumb for selecting $\Delta t$ is:

$$
\Delta t=\tau / 20
$$

in which $\tau$ is the period of the fastest motion. ${ }^{48}$

\subsubsection{Replica Exchange Molecular Dynamics}

A complex system like a protein with many moveable and interacting parts is likely to have a complicated energy landscape with many hills and valleys. Exploring such a landscape to find preferred, low enegy configurations with MD simulations can be difficult. The initial configuration is often chosen for reasons of convenience, but because of high barriers, the system may be unable to leave the initial location in configuration space if the temperature of the simulation is low. Conversely, if the temperature of the simulation is set very high, the system can quickly change its configuration from the initial one, but may never settle into any configuration because

high temperatures cannot distinguish potential energy minima. REMD $^{52,53,54}$ is used to allow the system to move around configuration space but also settle into a region that has a potential energy minimum. Because of this, REMD is known as a technique of enhanced sampling.

Replica Exchange Molecular Dynamics is performed by first creating many replicas of the initial configuration of the system and assigning each replica a different 
temperature. Each replica is independently but simultaneously simulated by MD. After a fixed number of MD time steps, a Metropolis-type test is applied to the replicas to decide whether to swap replicas at adjacent temperatures. The temperature exchange probability is given by $P_{i j}=\min \{1, \exp (-\Delta)\}$ with $\Delta=\left(E_{j}-E_{i}\right) *\left[1 /\left(k T_{i}\right)-1 /\left(k T_{j}\right)\right]$ and $E_{j}$ the potential energy of replica $j$. After this test for swapping is performed and the replicas that fit the criteria are temperature swapped, another fixed number of MD steps is performed on all replicas, followed by another test for swapping adjacent temperatures, and so on. During stages when a replica is at high temperatures, it is able to escape from configurational kinetic traps (energy minima) and sample large regions of structural configuration space, but is unlikely to settle into any specific, low energy configurations. However, a replica that finds a low energy configuration is likely to swap to a lower temperature and thus increase its probability to remain in a low energy configuration.

\subsubsection{Implicit Solvation}

The natural environment for a protein system is water. Explicitly including water atoms in addition to protein atoms is computationally expensive. Ninety percent or more of the computation time may be spent on water-water interactions, which are not directly relevant to the protein dynamics. In a protein system, often the water-water dynamics are not of major importance. To speed up the calculations, implicit solvents can been used in MD simulations. Chapter

7 in Computational Biochemistry and Biophysics ${ }^{48}$ gives general descriptions of 
different ways to represent the water solvent implicitly, rather than using explicit water molecules.

One such implicit solvation model is EEF1. ${ }^{55,40,56}$ It uses an excluded volume implicit solvation model and the CHARMM 19 polar hydrogen energy. ${ }^{49}$ It also implicitly models the dielectric screeing of water molecules by a distance-dependent dielectric function, $\varepsilon=r,{ }^{57}$ as well as neutralizing ionic sidechains. ${ }^{57,49}$ It also uses the solvent exclusion effect, which assumes that the solvation free energy of each group is equal to a reference solvation free energy of that group minus the solvation it loses due to solvent exclusion by other atoms around it. ${ }^{57}$

Strodel et al. performed REMD and basin hopping global optimization to study the KFFE monomer and dimer energy landscapes, using four implicit solvent models. ${ }^{40}$ They found that of the four models they tested (EEF1 and three generalized Born models) EEF1 provided the best description for KFFE when comparing to experimental results.

In order to model solvent friction and Brownian motion of the solvent, the Langevin dynamics method is often used with an implicit solvation model. The Langevin equation is:

$$
m_{i} \frac{d^{2} r_{i}}{d t^{2}}=-\nabla_{i} U(r)-m_{i} \beta v_{i}(t)+R_{i}(r)
$$

$\beta$ is the reduced friction coefficient. $R_{i}(t)$ is the random force representing stochastic collisions between solvent molecules and solute. 


\subsubsection{Confining Potentials and Periodic Boundary Conditions}

Periodic Boundary Conditions (PBC) can be used in MD simulations to decrease the volume of the system that is included in the simulations and thus allowing the simulations to run faster. ${ }^{49} \mathrm{~A}$ boundary is created in the shape of a cube. When an atom exits on the left side of the boundary, it enters on the right side of the boundary. The same thing occurs for the top and bottom edges of the $\mathrm{PBC}$ cube, as well as the front and back edges of the cube.

Another way to confine the system is by using a repulsive spherical potential to push the atoms back once they have encountered the confining potential.

\subsection{My Particular Molecular Dynamics Simulation}

To speed up the structural conversion process so that I could investigate the $\beta$ formation and aggregation dynamics, I performed an REMD simulation using the CHARMM EEF1.1 force field with the MMTSB toolset, ${ }^{58}$ using CHARMM version 35b2. EEF1.1 is the same as EEF1 mentioned above, except that EEF1.1 contains some adjustments in the parameters describing partial charges. The adjustments are empirical adjustments and are made because some of the parameters in the EEF1 force field model simulate the ionizable side chains too strongly. 
I used an MD step of $2 \mathrm{fs}$ and I had CHARMM write every 500 steps (making each frame 1ps apart). My REMD simulation checked every $500 \mathrm{MD}$ steps (every 1ps) for whether to swap temperatures between replicas of adjacent temperatures. I used exponential spacing of the temperatures with a range of $380 \mathrm{~K}-600 \mathrm{~K}$.

In order to make up for the Brownian motion and frictional effects of the solvent that are not simulated in the EEF1 implicit solvent, I used Langevin Dynamics with a reduced friction coefficient of 1 .

The proteins were confined by a spherical quartic potential that had the value zero for $r<D R O F F$ and a value of Force $* \Delta^{2} *\left(\Delta^{2}-P 1\right)$ for $r>D R O F F$ with $\Delta=r-D R O F F$. I used Force $=0.2$ and $P 1=2.25$. A DROFF of $600 \AA$ was used to minimize the effect of the confining potential on the aggregation process.

The number of interchain and intrachain hydrogen bonds were calculated using Visual Molecular Dynamics. Many of the parameters were calculated using Python programs analyzing large PDB files containing the coordinates of each atom during each frame. 


\section{MOLECULAR RATE CONSTANTS FOR AMYLOID $\beta$ FIBRILLOGENESIS}

An extensively studied, naturally occurring peptide system is the amyloid beta precursor $\mathrm{A} \beta$ peptide, which is responsible for amyloid deposits in $\mathrm{AD} .^{59}$ On the basis of experimental data, I have developed a mathematical model for the reaction pathways and determined rate parameters for peptide secondary structural conversion and aggregation during the entire fibrillogenesis process from random coil to mature fibrils, including the molecular species that accelerate the conversions. The model and the rate parameters include different molecular structural stages in the nucleation and polymerization processes and the numerical solutions yield graphs of concentrations of different molecular species versus time that are in close agreement with experimental results. My model also allows for the calculation of the time-dependent increase in aggregate size.

In Section 3.1, I give a general description of $A \beta$ Proteins. Section 3.2 describes the various structures in my model. In Section 3.3, I describe model details and the results of fitting my model to experimental data.

\subsection{Description of $A \boldsymbol{\beta}$ Proteins}

A protein that is produced normally in the human body throughout life is $A \beta$. It is not known what function the $A \beta$ protein in humans has, if any. It is cleaved from a larger precursor protein, $\mathrm{A} \beta \mathrm{PP} . \mathrm{A} \beta$ amyloids in the neuropil are associated with $\mathrm{AD}$.

The primary structure of $A \beta$ is:

DAEFRHDSGYEVHHQKLVFFAEDVGSNKGAIIGLMVGGVVIA. 
Naturally occurring A $\beta$ peptides have a length in the range of 39-43 amino acids. Experiments have elucidated valuable structural information of variants such as $A \beta(10-$

35), $A \beta(1-40)$ and $A \beta(1-42)$ peptides. ${ }^{60,61,62}$ The predominant forms of $A \beta$ produced in vivo are $A \beta(1-40)$ and $A \beta(1-42)$.

Different segments of $A \beta$ have different properties. For instance, $A \beta(1-40)$ has $48 \%$ hydrophobic residues and 40\% hydrophilic residues whereas $A \beta(16-22)$ has $71 \%$ hydrophobic residues and 29\% hydrophilic residues. Different segments within A $\beta(1-$ 40) have different secondary structure propensities. ${ }^{63}$

\subsection{Description of Various Structures in My Model}

After dissolution, $\mathrm{A} \beta$ proteins tend to exist as monomers, dimers, and very small oligomers of mostly unstructured random coil and $\beta$-turn peptides, along with a small amount of structured $\beta$-strands and $\beta$-sheets. They then form micelles, which are loosely bound disordered aggregates. These micelles then break apart into nuclei. The nuclei are small and reactive to proteins and brain cells. They are $\beta$-aggregates and grow by forming with other nuclei and having monomers attach to them. When they have these monomers attach to them, they tend to catalyze the added proteins to the $\beta$ strand state. These nuclei continue to grow until they are large enough to be called protofilaments.

Protofilaments are composed of $\beta$-sheets parallel to each other. Protofilaments wrap around each other like strands of rope to make protofibrils. Protofibrils are soluble and can grow either by attaching of single monomers or attaching of all kinds of $\beta$ - 
aggregates. When protofibrils are large enough they become fibrils, also called mature fibrils.

The description above is a simple one. However, in real A $\beta$ proteins, there are many different oligomers and pathways toward fibrils. ${ }^{9}$ Assuming a simple process toward aggregation allowed me to be able to define a functional model for $\mathrm{A} \beta$ fibrillation.

\subsection{Results}

The role of fibrillar $\mathrm{A} \beta$ in $\mathrm{AD}$ has stimulated interest in the kinetics of $\mathrm{A} \beta$ fibril formation. Determination of rate parameters for the various peptide structural conversion steps during fibril formation may be helpful in developing efficacious therapies for AD. Various research groups have been successful in performing very difficult experiments.

Kinetic models that include reaction pathways and rate parameters for the various stages of the process can be helpful toward understanding the dynamics on a molecular level. Rates for peptide structural transformations that nucleate $\beta$-structure, as well as rates for aggregation/polymerization into mature fibrils are especially interesting. There are experimental data on rate parameters for some of the stages of the fibril formation process. These include the investigations on the elongation and nucleation rate parameters for fibrillogenesis at low $\mathrm{pH},{ }^{2}$ rates of nuclei production and association, ${ }^{64}$ and lag time and rate of aggregation. ${ }^{65}$ Kinetic rate equations were used by Pallitto and Murphy ${ }^{7}$ to model folding and aggregation. Kinetic models can further benefit from experiments that have shed light on aspects of the process of $A \beta$ 
aggregation from random coil to mature fibril. Kirkitadze et al. ${ }^{28}$ were able to measure the temporal changes of concentrations of different types of secondary structures:

random coil, $\alpha$-helix, $\beta$-strand/sheet, and $\beta$-turn for both $A \beta(1-40)$ and $A \beta(1-42)$. These measurements were done over a period of 20 days for $A \beta(1-40)$ and 10 days for $A \beta(1$ 42), allowing enough time for fibrillogenesis to occur. Fezoui and Teplow ${ }^{36}$ measured the concentration of prefibrillar and fibrillar $\beta$-structure as a function of time in different solvent conditions.

Determination of rate parameters for the various peptide structural conversion steps during fibril formation may be helpful in developing efficacious therapies for AD. The numerical solutions of my model yield graphs of concentrations of different molecular species versus time. With the proper choice of transition rate parameters, my model is able to nicely fit the time dependence of the concentrations measured experimentally by Kirkitadze et al., ${ }^{28}$ Walsh et al., ${ }^{37}$ and Fezoui and Teplow. ${ }^{36}$ My model and the rate constants include different molecular structural stages in the nucleation and polymerization process and provide insight on the molecular species involved in the fibril formation process.

\subsubsection{Model of Reaction Pathways and Experimental Data}

On the basis of the experimental and computational results, Bitan et al. ${ }^{66}$ hypothesized that hydrophobic residues on different helical peptides might aggregate to create a paranucleus, which can then transform to amyloid protofibrils. In addition, it has been suggested that the process of structural conversion may be accelerated by the presence of prefibrillar $\beta$-aggregates of varying sizes. ${ }^{33,67}$ Experimental data ${ }^{28,2,37}$ 
suggest that the population of the $\alpha$-helix species increases rapidly (and non-linearly) as the $\beta$-structure begins to appear near the end of a lag phase. The lag phase (also called lag time) is the time it takes for a significant number of nuclei to form. During the lag phase, not much structural conversion takes place, which shows that the appearance of $\beta$-species plays a role in the structural conversion. Although a detailed molecular description of the nucleation mechanism is still not fully understood, Auer et al. ${ }^{68}$ suggest that the surface of a growing $\beta$-sheet acts as a substrate for the attachment of $\alpha$ helical peptides during the aggregation process. Therefore, I assumed that the random coil $\rightarrow \alpha$-helix transition is affected by the presence of $\beta$-strands. Some experiments ${ }^{69,70}$ suggest that fibrillogenesis proceeds from random coils directly to $\beta$-structure. The prefibrillar structures can serve as toxic elements that facilitate the structural conversion from random coil to $\alpha$-helix, and also in the conversion from $\alpha$-helix to $\beta$-structure.

Using differential equations for studying the change in protein compartments with time is described in Chapter 4 of Protein Folding Kinetics by Nolting. ${ }^{44}$ Nolting shows coupled differential equations that are simple enough to have analytic solutions. The differential equations in Protein Folding Kinetics do not take into consideration the heterogeneity in the rate constants. The set of differential equations for my model is more complicated.

On the basis of experimental observations described in an article by Kirkitadze et al., ${ }^{28}$ I made the following assumptions in formulating my reaction pathway model: (i)The conformational transitions which occur during fibrillogenesis proceed as random coil $\rightarrow \alpha$-helix $\rightarrow \beta$-strand/sheet $\rightarrow \beta$ aggregate/protofibril $\rightarrow$ mature fibril, (ii)the processes of random coil $\rightarrow \alpha$ - 
helix, and $\alpha$-helix $\rightarrow \beta$-strand conversion are accelerated by the presence of toxic $\beta$-strand aggregates, (iii) random coil/ $\beta$-turn structures incorporated in the mature fibril state retain their unstructured properties.

With these basic assumptions, I developed a kinetic, state-transition model (Figure 3.1) in which the amino acid configurations are grouped into various stages during the fibril formation process. I used differential equations to study the changes in population in these secondary structure configurations: random coil/ $\beta$-turn, $\alpha$-helix, $\beta$-strand/sheet. Each compartment represents the percentage of amino acids from all the proteins in the system with a characteristic secondary structure. Many of the compartments also had another characterstic in addition to the secondary structure, as described below.

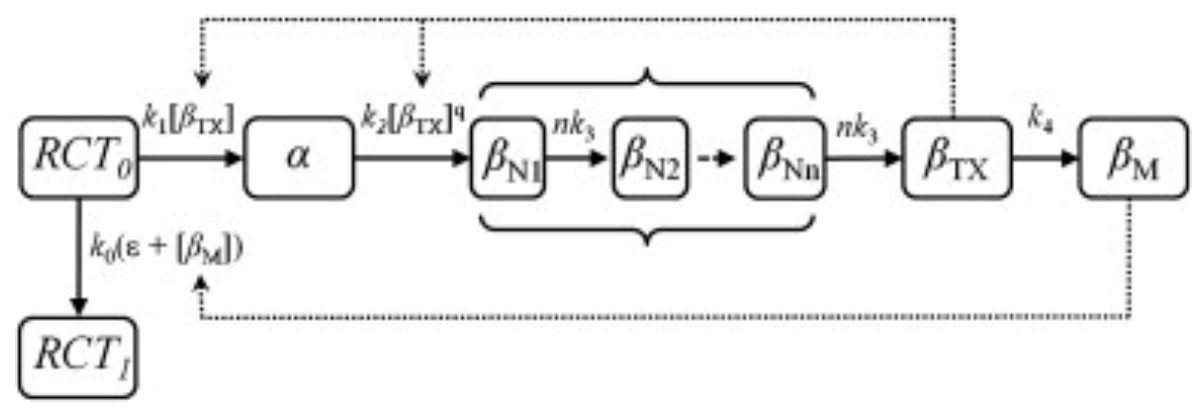

Figure 3.1 A state transition model of the structural conversion process of the $\mathrm{A} \beta$ formation process. To simulate the heterogeneity in $\beta_{N}$ structure, the $\beta_{N}$ phase is treated purely mathematically as a series of $n$ fictitious subcompartments. For all sub-compartments, the rate of transition to the next subcompartment is given the same value, $n k_{3}$. The reason for this is given in detail below.

The notation for the species and kinetic parameters included in the schematic diagram of Figure 3.1 are explained in Table 3.1. 
$R C T_{0} \quad$ Initial combined concentration of random coil $/ \beta$-turn

$R C T_{1} \quad$ Fraction of inital concentration of random coil $/ \beta$-turn that retain the random coil structure and are either directly incorporated into mature fibrils or remain isolated as monomers/dimers

$\alpha \quad \alpha$-helix concentration

$\beta_{N} \quad$ Concentration of non-toxic $\beta$-strands/sheets/aggregates such as micelles

$\beta_{T x} \quad$ Concentration of $\beta$-strand aggregates such as protofibrils

$\beta_{M} \quad$ Concentration of larger, non-toxic $\beta$-aggregates such as mature fibrils

$k_{0}, k_{1}, k_{2}, k_{3}$, Rate parameters for transitions from one structural state to another $k_{4}$

$\varepsilon \quad$ Fraction of initial monomers/dimers that are stable and do not participate in fibrillogenesis

Table 3.1 Parameters Used in This Model.

The differential equations used to quantify my model presented in Fig 3.1 are equations (3.1)-(3.8).

$$
\begin{aligned}
& \frac{d\left[R C T_{0}\right]}{d t}=-k_{0}\left[\beta_{M}\right]\left[R C T_{0}\right]-k_{1}\left[\beta_{T X}\right]\left[R C T_{0}\right] \\
& \frac{d[\alpha]}{d t}=k_{1}\left[\beta_{T X}\right]\left[R C T_{0}\right]-k_{2}\left[\beta_{T X}\right]^{q}[\alpha] \\
& \frac{d\left[\beta_{N 1}\right]}{d t}=k_{2}\left[\beta_{T X}\right]^{q}[\alpha]-n k_{3}\left[\beta_{N 1}\right] \\
& \frac{d\left[\beta_{N 2}\right]}{d t}=n k_{3}\left[\beta_{N 1}\right]-n k_{3}\left[\beta_{N 2}\right] \\
& \vdots \\
& \frac{d\left[\beta_{N n}\right]}{d t}=n k_{3}\left[\beta_{N n-1}\right]-n k_{3}\left[\beta_{N n}\right]
\end{aligned}
$$




$$
\begin{aligned}
& \frac{d\left[\beta_{T X}\right]}{d t}=n k_{3}\left[\beta_{N n}\right]-k_{4}\left[\beta_{T X}\right] \\
& \frac{d\left[\beta_{M}\right]}{d t}=k_{4}\left[\beta_{T X}\right] \\
& \frac{d\left[R C T_{1}\right]}{d t}=k_{0}\left[\beta_{M}\right]\left[R C T_{0}\right]
\end{aligned}
$$

Summing the right side of all the equations (3.1)-(3.8) gives zero because my model had no proteins entering or leaving, and the differential equations are consistent with the mass action principle.

Figure 3.1 and equations (3.1)-(3.8) represent a model with the following details. An initial combined concentration of random coil/ $\beta$-turn configurations is represented by $R C T_{0}$. A fraction of the $R C T_{0}$ molecules remain in the random coil configuration and are denoted as $R C T_{1}$. The $R C T_{1}$ molecules are composed of two types. One component of $R C T_{1}$ contains molecules that undergo rapid and irreversible commitment to stable monomers/dimers, as found experimentally in Pallitto and Murphy, ${ }^{7}$ and represented by the path with rate constant $k_{\mathrm{o}} \varepsilon$.

The other contribution to the population of $R C T_{1}$ is from $R C T_{0}$ amino acids that are either part of a mature fibril or sequestered in a mature fibril. The process of $R C T_{0}$ amino acids becoming either part of a mature fibril or sequestered in a mature fibril requires mature fibrils and its rate depends upon $\left[\beta_{M}\right]$. The experimental results in Fig. $1 \mathrm{~b}$ of Kirkitadze et al. ${ }^{28}$ show that the combined random coil/ $\beta$-turn content levels off at long time scales, which implies that as the process of structure conversion proceeds and prefibrillar and 
fibrillar structures start to form, some initial random coil/ $\beta$-turn components retain their structures and may never convert to $\alpha$-helix. Another study ${ }^{37}$ found significant amounts of random coil/ $\beta$-turn incorporated into both protofibril and fibril structures. Since mature fibrils are stable, the random coil/ $\beta$-turn components in these structures can no longer transform to other structures. ${ }^{63,71,72}$

The fraction of $R C T_{0}$ that does not convert to $R C T_{1}$ follows the main pathway for fibrillogenesis in Fig. 3.1 and transforms into $\alpha$-helix secondary structure configuration with a rate $k_{1}$, and this process is accelerated by the toxic $\beta$-aggregate species that are grouped in $\beta_{T X}$. The $\beta_{T X}$ compartment includes toxic species of varying sizes, including small aggregates.

There are two important issues that must be explained by the model: determining a molecular mechanism that generates the lag phase observed in the experiments, and determining a reaction pathway mechanism to correctly reproduce a rapid drop-off at long times in the concentration of $\alpha$-helix. I first address the issue of a molecular explanation of the lag phase.

\subsubsection{Modeling of the Lag Phase}

For the specific experiments that are analyzed in my work, there is little change in the concentration of any of the different species during the first four days of observation. Circular dichroism measurements revealed that the $A \beta(1-$ 40) oligomerization was preceded by an increase in $\alpha$-helical content from $3 \%$ to $>30 \%$. The $\alpha$-helix content begins to rise after four days of incubation 
whereas the fibrils are observed only after six days. Similar results from MD simulations were obtained by Bumketner et al., ${ }^{73}$ in which a modest $\alpha$-helical content was observed.

The sub-compartmentalization of the $\beta_{N}$ phase is introduced to explain this lag phase. The series of $\beta_{N}$ compartments is used as a mathematical method to represent a single phase that has heterogeneous structures with a wide range of rate constants. If a single $\beta_{N}$ compartment is used, representing a relatively homogeneous population, there is no initial delay in change in concentrations. Mathematically, if we ignored the heterogeneity in $\beta_{N}$ by using only a single $\beta_{N}$ compartment with a single rate constant $k_{3}$, no lag phase occurs because $\beta$ strand/sheet structures that are included in the initial preparation as $\beta_{N}$ are allowed to convert to toxic protofibrils $\left(\beta_{T X}\right)$ with a single rate constant $k_{3}$ and the resulting $\beta_{T X}$ accelerates the $R C T_{0} \rightarrow \alpha$-helix conversion process. A single non-toxic $\beta_{N}$ compartment is equivalent to the assumption of a homogeneous collection of $\beta_{N}$ structures, all with the same value of $k_{3}$. However, $\beta_{N}$ aggregates may contain a range of sizes or geometries. This structural heterogeneity ${ }^{74}$ complicates the conversion process and produces a distribution in the transition rate from $\beta_{N} \rightarrow \beta_{T X}$. This structural heterogeneity is the molecular basis for explaining the lag phase and is incorporated into my model.

The effects on the kinetics of a single process due to a heterogeneous distribution in reaction rate constants can be mathematically simulated in a model by incorporating a series of ' $n$ ' fictitious steps (Fig. 3.1). The method of multiple stages, wherein a single compartment is broken into several identical 
compartments in series, is widely used in epidemiological models. ${ }^{75,76,77}$ Each sub-compartment ' $i$ ' acts as a homogeneous collection of molecules. In order for the average time for the process to remain as $\tau=1 / k$, all sub-compartments have the same mean waiting time, $\tau_{i}^{\prime}=\tau / \mathrm{n}=1 / n k$ and the same rate parameter $k^{\prime}=n k$. The resulting average total time spent in all $n$ sub-compartments is just $\tau=n \tau^{\prime}{ }_{i}=n(1 / n k)=1 / k$, the same average total time as if it was a single compartment with rate constant $k$. Though the average time to transition from $\beta_{N} \rightarrow \beta_{T X}$ is the same whether there is a single compartment with a single rate parameter $k_{3}$, or a series of $n$ fictitious compartments (as in the model), each with rate parameter $n k_{3}$, the probability distribution of individual molecular reaction times is not the same.

The probability that a random variable is between two values is the definite integral of the probability function between these two values. For the simple process of entities transforming from compartment $A$ to compartment $B$ with a single rate parameter, the probability density function for molecular reaction times is exponential, as shown in Fig 2.8.

For a series of compartments in my model, the probability density distribution is described by a gamma function. The sub-compartmentalization method generates the same distribution in reaction times as expected from a heterogeneous collection of structures, each performing a single step conversion at a different rate. The probability density function for $\beta_{N} \rightarrow \beta_{T X}$ reaction times $p(t)$ is given by $p(t)=t^{n-1} e^{-n k t}\left[(n k)^{n} / \Gamma(n)\right]$, where $\Gamma(n)$ is the gamma function, and $n$ is the number of sub-compartments. The variance in reaction rates of this 
distribution is given by $1 /\left(n k^{2}\right)$. The variance of this distribution is manifested on a macroscopic experimental level by producing a lag phase in the aggregation process. The number of sub-compartments in the model can be estimated by fitting the experimentally observed lag phase.

The gamma distribution of reaction times for the $\beta_{N}$ structures is more in alignment with what is known about the molecular species. The $\beta_{N}$ species undergoes aggregation as it transforms into the toxic $\beta_{T X}$ species. The dynamics of aggregation are complex with many different molecular routes, and therefore it is expected that aggregation of $\beta$-strands $/ \beta$-sheets into protofibrils is not a homogeneous process. In light of this, we can view the mean reaction time as a mean aggregation time. Interestingly, the lack of lag phase with the use of a single compartment, homogeneous $\beta_{N}$ resembles the faster nucleation and

polymerization processes observed for $\mathrm{A} \beta(1-42)^{78,28}$ which displays a significantly shorter lag time as compared to $A \beta(1-40)$. I focus on $A \beta(1-40)$ because there are more experimental data points than for $A \beta(1-42)$.

\subsubsection{Modeling the Rapid Drop-Off at Long Times in $\alpha$-Helix}

\section{Concentration}

Another important aspect incorporated into my model is the ability of $\beta_{T X}$ to accelerate the process of $\alpha$-helix $\rightarrow \beta$-strand conversion in order to have the theoretical $\alpha$-helix curve drop off at long times as quickly as the experimental data in the tail after the peak. In order to match the quick drop observed experimentally, the degree of this dependence had to be enhanced by 
introducing the exponent $q$ in the $\beta_{T X}$ activity so that the rate of conversion from $\alpha$-structure is given by $k_{2}\left[\beta_{T X}\right]^{q}$. Because of the complex nature of the aggregation process, a higher-order concentration dependence $(q>1)$ is not unusual.

\subsection{Numerical Results Using My Model to Fit the Experimental Data}

I developed my kinetic model to explain the experimental data on the $\mathrm{A} \beta$ fibrillogenesis process from several studies. In one study, Kirkitadze et al. ${ }^{28}$ experimentally followed the time course of the fibril formation process and measured the percentages of various structural elements for both $A \beta(1-40)$ and $A \beta(1-42)$ peptides. I fit my theoretical curves to the experimental data for $A \beta(1-40)$ fibrillogenesis

presented in Fig. $1 \mathrm{~b}$ of Kirkitadze et al. ${ }^{28}$ The Kirkitadze data are reproduced as the points in Fig. 3.2.

In order to monitor the development of various secondary structural motifs during fibrillogenesis, they initialized their $A \beta$ peptide samples by subjecting them to a dissolution process. This dissolution process produced a starting solution of mostly unstructured random coil and $\beta$-turn peptides, along with a small amount of structured $\beta$-strands and $\beta$-sheets. Over a period of three weeks, the solution was subjected to circular dichroism (CD) analysis to track the time evolution of random coil, $\beta$-turn, $\alpha$-helix, and $\beta$-strand concentrations. Accurate estimation of the $\beta$-turn content was relatively more difficult than accurate estimation of $\alpha$-helix and $\beta$-strand concentrations as a result of the $\beta$ - 
turn's structural variations. Two different deconvolution algorithms for spectral analyses $\left(\mathrm{CDANAL}^{79}\right.$ and CONTIN/LL $\left.{ }^{80}\right)$ yielded the same results for the concentrations of both $\alpha$-helix and $\beta$-strand but yielded quite different $\beta$-turn content. However, the sum of the random coil and the $\beta$-turn concentrations remained the same using either method. Since both methods give the same results for the combined concentrations of random coil and $\beta$-turn, my model treats them together.

The rate parameters for the aggregation kinetics depend on factors such as $\mathrm{pH}$, initial concentrations, temperature, ${ }^{81}$ and methods of preparation for the initial protein sample. ${ }^{28,3,81}$ Therefore, lag times vary greatly among experiments. ${ }^{13,81,82}$ Lag time is highly dependent on initial concentrations among the different species as it is sensitive to various assembly states of the $\beta$ aggregates. $^{83}$

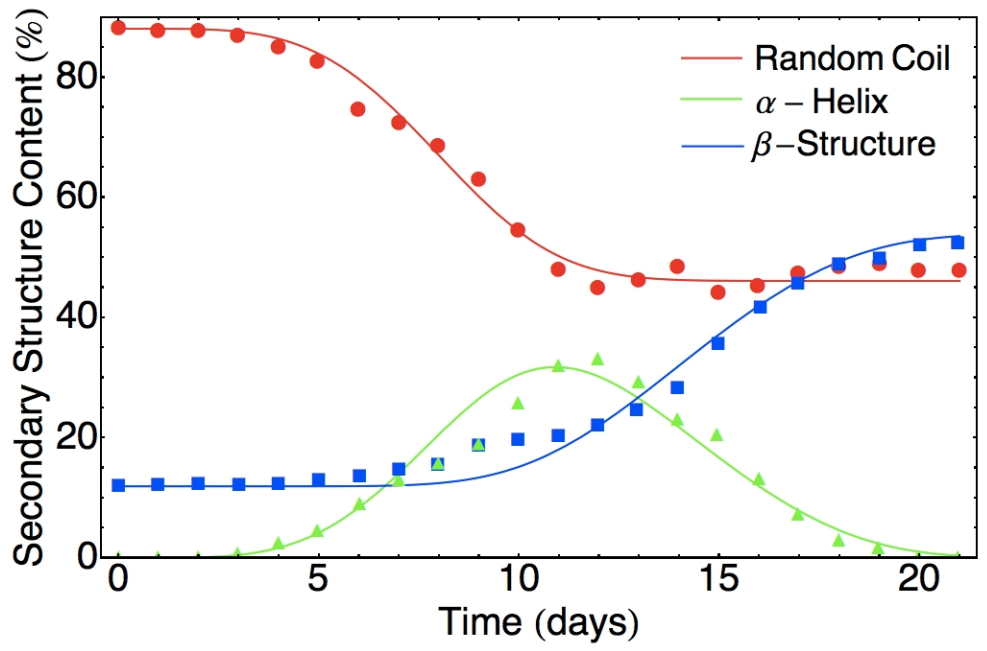

Figure 3.2 Experimental data (points) from Kirkitadze et al. ${ }^{28}$ fit with theoretical curves derived from the model of Fig. 3.1. 
Following the experimental conditions, I set the initial contents to be $R C T_{0}(t=0)=88.1 \%, \beta_{N 1}(t=0)=11.9 \%$, and zero for all other compartments. The concentration of the monomers/dimers is difficult to measure, and quantitative information is not available. ${ }^{7}$ Therefore I set $\varepsilon=0$ in my work. The parameter $\varepsilon$ can be appropriately adjusted when experimental data are available. The model includes the reaction pathway with sub-compartments that generate a time lag that results from nucleation and the resulting curves accurately fit the data.

In Figure 3.2, I display my fit to the experimental data presented in Kirkitadze et al. ${ }^{28}$ The following values of the rate parameters generated the curves: $k_{0}=0.590 /$ day, $k_{1}=0.672 /$ day, $k_{2}=0.678 /$ day, $k_{3}=0.0392 /$ day, and $k_{4}=0.554 /$ day. The number of subcompartments is $n=4$, and the exponent is $q=2$. From Fig. 3.2, it is clear that my model accurately fits the experimental data. It successfully reproduces the lag phase kinetics involved in the fibril formation process. My model also reproduces the sharp drop in the experimental $\alpha$-helix curve near the end of the process.

I further use my model to fit other experimental data. Specifically, I fit the experimental data by Walsh et al. ${ }^{37}$ and by Fezoui and Teplow. ${ }^{36}$ Walsh et al. ${ }^{37}$ performed experiments with the low molecular weight $A \beta(1-40)$ peptide and followed the conformational changes during the fibril formation process with $\mathrm{CD}$ spectra for over 30 days. The data points in Fig. 3.3a show the secondary structure content plotted against time as given in Table I of Walsh et al. ${ }^{37}$ Their data are also consistent with similar observations that fibrillogenesis involves formation of intermediate $\alpha$-helix secondary structure during the conformational transition from random coil to $\beta$ aggregates. The curves in Fig. 3.3a show the fit with my model. Following the 
experiments, I set the initial contents to be $R C T_{0}(t=0)=76 \%, \alpha(t=0)=11 \%$, $\beta_{N 1}(t=0)=13 \%$, and zero for all other species and compartments. The transition rates and other parameters that generated my fitting curves were $k_{0}=0.0154 /$ day, $k_{1}=0.0318 /$ day, $k_{2}=0.0282 /$ day, $k_{3}=0.0565 /$ day, $k_{4}=0.520 /$ day, and the number of sub-compartments $n=6$. I note that different experimental conditions will affect the rates of different steps in the process. For example, though the temporal trends are similar, the data from Walsh et al. ${ }^{37}$ display much smaller changes in concentrations than the data from Kirkitadze et al. ${ }^{28}$ because of differences in experimental conditions. Therefore, the values for the rate parameters used in the fit in Figure 3.3a are different than those used for the curves in Figure 3.2. The exponent $q=2$ remains the same as used for the curves in Fig. 3.2. 

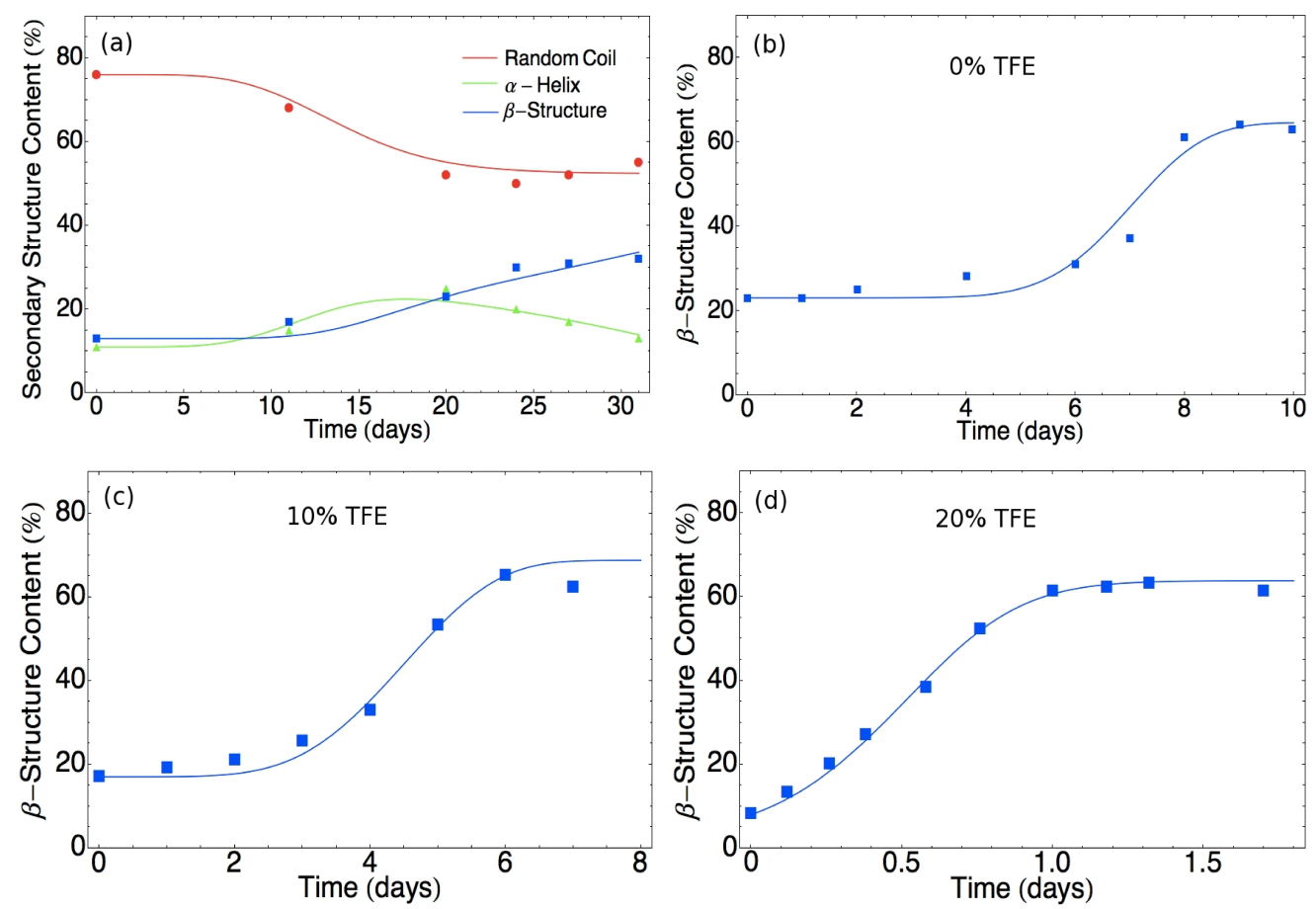

Figure 3.3 (a) The experimental data points from Walsh et al. ${ }^{37}$ showing the secondary structure content obtained by deconvolution of CD spectra. The curves are the numerical results from fits obtained using my model. (b-d) Data points from Fezoui and Teplow ${ }^{36}$ showing the temporal changes in the $\beta$-structure content for $A \beta(1-40)$ fibrillogenesis at different TFE concentrations.

\subsubsection{Co-Solvents}

Experimentalists often use co-solvents with water, which changes the fibril formation dynamics as compared to a sytem of proteins in pure water. Urea is

commonly used to denature proteins. ${ }^{84}$ Alcohols are often used as co-solvents. ${ }^{84}$ A good review of alcohol co-solvents is given by Buck. ${ }^{85}$

Trifluoroethanol (TFE) is a fluorinated alcohol. Proteins in TFE/water solution having different secondary structure dynamics than when in a pure water solvent. ${ }^{86}$ The effects of TFE seem to depend on amino acid sequence. ${ }^{85}$ It is well established that TFE promotes formation and stabilzation of $\alpha$-helices and hairpins. ${ }^{87} \mathrm{~A}$ theory as to why 
TFE favors the formation of intrachain hydrogen bonds is that TFE molecules coat the outside of a protein. ${ }^{86}$ The coating displaces water molecules ${ }^{86}$ which removes hydrogen bonding partners to which backbone atoms could hydrogen bond. ${ }^{86}$ The displacement of water also creates a lower dielectric environment which promotes electrostatic interactions. ${ }^{86}$ Water has one of the largest dielectric constants among fluids, 78 at $25^{0} \mathrm{C} .{ }^{88}$ Trifluoroethanol does not does not disrupt severely the hydrophobic interactions within a protein ${ }^{86}$ or may even strengthen them, which is in contrast with most other organic solvents.

However, it is not well established whether TFE promotes or inhibits formation of $\beta$-sheets. Trifluoroethanol has been known to disrupt and also enhance quaternary interactions. The hydrophobic interactions between proteins tend to be weakened by TFE. The TFE molecule is approximately nine times larger than the water molecule.

Circular Dichroism spectropolarimetry was done on T4 LYS (11-36), a peptide dervied from a region of the T4 lysozyme molecule, by Anderson et al. ${ }^{89}$ It was found that as the TFE content rose to $20 \%$, the $\beta$-sheet content grew from 26 to $38 \%{ }^{89}$ However, as the TFE content then rose to $50 \%$, the $\beta$-sheet content decreased to $15 \%{ }^{89}$ In terms of the energy landscape picture of Fig. 3.4, TFE lowers the energy of the $\alpha$-helix state. In addition, the height of the barriers for the $\mathrm{RC} \rightarrow \alpha$-helix transition and the $\alpha$-helix $\rightarrow \beta$-structure transition can be affected by TFE, as evidenced by the temporal data in Fig. 2 a of Fezoui and Teplow. ${ }^{36}$

Fezoui and Teplow ${ }^{36}$ show that the amount of $A \beta$, the protein whose fibrils cause $\mathrm{AD}$, goes down with increasing TFE concentration and is 0 for $30 \%$ and higher TFE concentration. ${ }^{36}$ It also shows increasing amounts of $\alpha$-helix with increasing TFE 
concentrations. ${ }^{36}$ However, the fibrils actually form faster when the TFE concentration is raised up to about $20 \%$. Experiments have found that the random coil to fibril formation process involves significant $\alpha$-helix intermediates for $A \beta$ and that the formation of these $\alpha$-helix intermediates is "on pathway" for fibril formation. ${ }^{90,28}$ Insight into the role of the $\alpha$-helix intermediate in the fibrillization process is provided by experiments ${ }^{36}$ that use TFE which facilitates and stabilizes helical structure. At TFE concentrations below $20 \%$, the fibril formation process is accelerated by increasing the concentration of TFE. However, the addition of higher concentrations of TFE can potentially slow fibril formation, implying that the $\alpha$-helix intermediate ${ }^{91}$ becomes too stable and does not allow the progression to the $\beta$-structures necessary for fibril formation. The dependence of fibril formation on the concentration of TFE can be explained thermodynamically with the help of an energy landscape diagram. A schematic energy landscape representing these structural stages is shown in Fig. 3.4. Increasing the TFE concentration deepens the energy minimum corresponding to the $\alpha$ helix structure, which can facilitate the transition from random coil $\rightarrow \alpha$-helix, a necessary step in fibrillogenesis. At low TFE concentrations, fibrillogenesis continues with the transition from $\alpha$-helix $\rightarrow \beta$-strand. However, at high TFE concentrations, the $\alpha$ helix structure becomes stabilized so strongly that the $\alpha$-helix energy minimum is difficult to escape. The $\alpha$-helix $\rightarrow \beta$-strand conversion is impeded and fibril formation is hindered. 


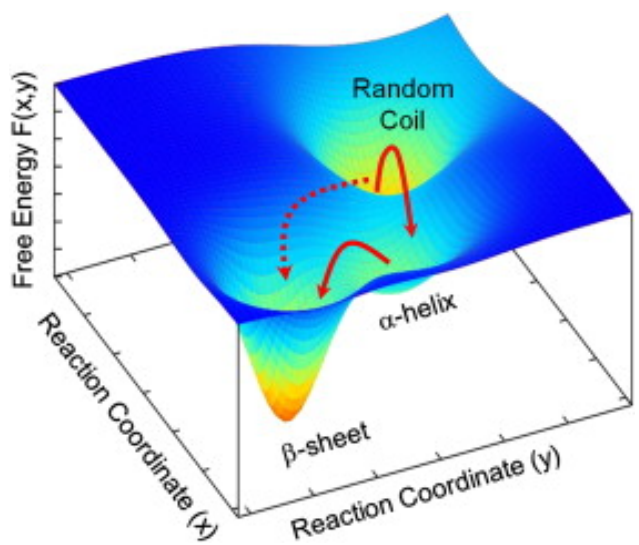

Fig. 3.4 Schematic energy landscape of $A \beta$ secondary structure conversion as a function of arbitrary structural reaction coordinates $x$ and $y$. TFE stabilization of $\alpha$-helix structure is represented by increasing the depth of the $\alpha$-helix energy minimum. If the $\alpha$-helix minimum is too deep, the peptide may find it difficult to undergo the conversion of $\alpha$-helix $\rightarrow \beta$-strand. The dotted arrow represents a possible pathway in which the random coil converts directly to $\beta$-structure, bypassing $\alpha$-helical structure.

Figure 3.3b-d shows the experimental data points from Fezoui and Teplow, ${ }^{36}$ which investigated the effects of $\alpha$-helix stabilization on $A \beta$ fibrillogenesis. In that work, Fezoui and Teplow used several different concentrations of helix stabilizing TFE solution and followed the rates of fibril formation and fibril elongation. They concluded that the helical intermediate structures play a crucial role in peptide oligomerization, which is followed by the conformational reorganization into extended $\beta$-sheets. Without TFE ( $0 \%$ concentration), the time course of $\beta$-structure content as shown in Figure $3.3 \mathrm{~b}$ is similar to that displayed in Figure 3.2.

Since Figure 3.3b-d only contains data for one molecular structural component, it may be possible to fit the data using different parameter sets because the fits are not constrained as strongly as for the data in Figure 3.2 and Figure 3.3a. Since the experimental conditions were significantly different in Figure 3.3b-d, I expect that the fit parameters may also be different. For example, the data in Figure 3.3b-d result from 
different initial concentrations of $\alpha$-helix. Differences in initial conditions, temperature, and TFE concentration lead to important differences in the time required before the $\beta$ structure content starts to dramatically increase and this will have a strong effect on the values of some of my model's parameters.

When the TFE concentration is increased up to $20 \%$, the fibril formation process is accelerated and the lag phase is decreased. For the condition of $0 \%$ TFE in Figure $3.3 \mathrm{~b}$, the fit parameter values are $k_{0}=3.00 /$ day, $k_{1}=0.448 /$ day, $k_{2}=0.310 /$ day, $k_{3}=0.0512 /$ day, $k_{4}=0.107 /$ day, $q=2$, and $n=4$. The fit parameters for $10 \%$ TFE in Fig. $3.3 \mathrm{c}$ are $k_{0}=1.23 /$ day, $k_{1}=0.463 /$ day, $k_{2}=0.350 /$ day, $k_{3}=0.100 /$ day, $k_{4}=0.733 /$ day, $q=2$, and $n=3$. For $20 \%$ TFE in Fig. 3.3 d, they are $k_{0}=0.771 /$ day, $k_{1}=0.0116 /$ day, $k_{2}=0.467 /$ day, $k_{3}=0.774 /$ day, $k_{4}=1.00 /$ day, $q=2$, and $n=1$. For Fig. $3.3 \mathrm{~b}-\mathrm{d}$ the initial content of $R C T_{0}$ and $\alpha$-helix were set to the values given in Table 1 of Fezoui and Teplow. ${ }^{36}$ The initial $\beta$ content that I used was determined from the data plots in Figure 2a of Fezoui and Teplow ${ }^{36}$ and divided as follows for the calculations in this paper: Figure $3.3 \mathrm{~b}\left(0 \%\right.$ TFE): $\beta_{N 1}(t=0)=18.1 \%, \beta_{N 2}(t=0)=5.0 \%$; Fig. $3.3 \mathrm{c}(10 \% \mathrm{TFE})$ : $\beta_{N 1}(t=0)=15 \%, \beta_{N 2}(t=0)=2.0 \%$; Fig. $3.3 \mathrm{~d}\left(20 \%\right.$ TFE): $\beta_{N 1}(t=0)=7.0 \%, \beta_{T X}(t=0)=1.0 \%$. All other species and compartments were assigned initial contents of $0 \%$.

My model fits the lag phase for various TFE concentrations up to $20 \%$ by adjusting the number $n$ of sub-compartments. The data for $0 \%$ TFE concentration have the longest lag phase ( $\sim 7$ days) and the largest number of sub-compartments $(n=4)$.

Similarly, $10 \%$ TFE requires $n=3$, and $20 \%$ TFE which has the smallest lag phase and fastest fibril formation has $n=1$. Increasing the TFE concentration enhances $\alpha$-helix 
stability as seen in Table 1 of Fezoui and Teplow ${ }^{36}$ and Buck et al ${ }^{85}$ Interestingly, the change in energy barrier heights causes an increase in the speed of the overall fibril formation process up to $20 \%$ TFE, but above this TFE concentration the increased stability of the $\alpha$-helical structure acts as a kinetic trap and slows down the overall process. In addition to the TFE concentration dependence, the lag phase also depends on $\mathrm{pH}$ of the solution, ${ }^{65,14,28}$ and my model can be adapted to these biochemical environments as well.

\subsection{Discussion of Results}

It is important to note that though my results provide a good fit to a variety of experimental data analyzed in this paper, my model may need modification for other experimental conditions. Both the $\alpha$-helix $\rightarrow \beta$-structure sequence of steps as well as the possibility of a process involving $\alpha$-helix $\rightarrow$ random coil $\rightarrow \beta$-sheet are discussed in the literature. $^{92,93}$ Velez-Vega and Escobedo ${ }^{94}$ discuss that the $\alpha$-helix stage may be more important for some peptides than for others, while Soto et al. ${ }^{91}$ and Szabo et al. ${ }^{95}$ discuss the possibility that the $\alpha$-helix secondary structure is not necessary for fibrillogenesis. Additional pathways for aggregates are also proposed. ${ }^{96}$ My model can be appropriately modified to reflect different pathways.

Important correlations can be made between my model and the kinetic model of

fibril nucleation and elongation developed by Lomakin et al. ${ }^{2}$ In that work, one pathway for the fibrillogenesis mechanism for high protein concentration was described as: Monomers $\longleftarrow \rightarrow$ Micelles $\rightarrow$ Nuclei $\rightarrow$ Fibrils. The pathway of Monomers $\longleftrightarrow$ Micelles $\rightarrow$ Nuclei $\rightarrow$ Fibrils is dominant when protein concentration 
exceeds a critical value above which pre-existing "seeds" are not necessary for fibril formation. Instead, high local protein concentration leads to formation of micelles that later form the domains of fibril nuclei. Experiments performed by Kirkitadze et al. ${ }^{28}$ and Lomakin et al. ${ }^{2}$ exhibit similar lag times even though they were performed at different peptide concentrations, $25 \mu \mathrm{M}$ and $>100 \mu \mathrm{M}$, respectively. The similarity may be due to their peptide concentrations being above their respective critical micellar concentrations at which the lag times become independent of the peptide concentration as observed. ${ }^{2,97}$ Other experiments ${ }^{13,14,15}$ as well as the nucleation theoretical treatment ${ }^{98}$ show a more complicated dependence of aggregation rate and lag time on peptide concentrations. The $A \beta$ monomers and the micelles were considered to be in rapid equilibrium so that this step was not the rate-limiting step. The two possible rate limiting steps in the model of Lomakin et al. ${ }^{2}$ are a micelle to nuclei formation process with a kinetic rate parameter they denoted as $k_{n}$, and a fibril elongation process with rate parameter $k_{e}$ that incorporates free monomers that are present with concentration $c$. Different nuclei sizes have been reported for different conditions. For example, Pellarin and Calfisch ${ }^{99}$ found the sizes of the nuclei to vary between 4 and 18 monomers corresponding to different micellar oligomeric conditions. This number can be $>30$ under certain conditions, as suggested by Garai et al ${ }^{74}$ Here I use the nuclei size to have 10 monomers as considered by Lomakin et al. ${ }^{2}$

The rate constant $k_{n}$ is the number of nuclei spontaneously formed out of micelles. ${ }^{2}$ By fitting the experimentally observed temporal evolution of the hydrodynamic radius of fibrils, Lomakin et al. ${ }^{2}$ estimated the value of $k_{n}$ to be $2.4 \times 10^{-6} \mathrm{~s}^{-1}$ or $0.207 \mathrm{~d}^{-1}$. Having $k_{n}$ be $0.207 \mathrm{~d}^{-1}$ represents one new nucleation every 
five days per micelle. My rate limiting step in Fig. 3.1, used to generate the curves in Fig. 3.2 and Fig. 3.3, involves a similar structural transformation. In order to compare with my model, I convert $k_{n}$ in order to represent the number rate at which monomers contained in micelles transition to monomers contained in nuclei, rather than the number of micelles that transition to nuclei. Since each micelle on average has an aggregation number of 25 monomers, whereas there is an average number of 10 monomers in a nucleus, I make the conversion as follows: $k_{n}=(0.207$ nuclei micelles $\left.^{-1} \mathrm{~d}^{-1}\right) \times(10$ monomers/nucleus $) /(25$ monomers $/$ micelle $)=$ $0.207 \mathrm{~d}^{-1} \times(10 / 25)=0.083 \mathrm{~d}^{-1}$. The comparable step in my model corresponds to amino acid residues in $\beta_{N}$ species transforming into residues in $\beta_{T X}$ species, and the rate is represented by $k_{3}$. Strikingly, the value of $k_{3}=0.039 \mathrm{~d}^{-1}\left(1.8 \times 10^{-6} \mathrm{~s}^{-1}\right)$ that I determined compares nicely (factor of 2) with Lomakin's value for $k_{n}$. Also, my value for $k_{3}$ falls nicely within the range of $k_{n}$ found experimentally by Wang et al. ${ }^{100}$

The agreement between my $k_{3}$ and the $k_{n}$ is further enhanced when we take into account that my value of $k_{3}$ was determined by fitting to experimental data obtained at $22{ }^{\circ} \mathrm{C}$, whereas experiments ${ }^{2}$ for $k_{n}$ were performed at $25^{\circ} \mathrm{C}$. Since these reactions would likely occur faster ${ }^{27}$ at higher temperatures, we would expect my $k_{3}$ to be larger if it was determined by analyzing experiments at higher temperature, if available. This would bring my $k_{3}$ even closer to $k_{n}$.

My $k_{3}$ and the nucleation rate $k_{n}$ refer to similar, but not identical processes. The observation that $k_{n} \geq k_{3}$ is consistent with my proposed fibrillogenesis mechanism. The parameter $k_{n}$ refers to nucleation, whereas my $k_{3}$ encompasses both nucleation and partial aggregation. Since nuclei must form before the formation of any toxic 
protofibrils, the rate of nucleation $k_{n}$ can be expected to be faster than $k_{3}$. Comparing my $k_{3}$ to Lomakin's $k_{n}$ directly connects Lomakin's model using macroscopic structures such as micelles and nuclei to my model, which deals with kinetics of microscopic secondary structural elements. I would also like to compare my rate parameters with the rate parameter for elongation of $k_{e}{ }^{2}$ However, comparing my rate parameters with the rate parameter for elongation of $k_{e}$ is especially complicated and requires additional work since fibril formation in my model finishes with the step $\beta_{T X} \rightarrow \beta_{M}$, but also includes a part of the $\beta_{N} \rightarrow \beta_{T X}$ conversion process.

My model also allows for the calculation of the time-dependent increase in aggregate size. I now show that the calculated results agree well with experimental results, and allow differences in experimental conditions to be included in the calculations. Fig. 2 of Lomakin et al. ${ }^{3}$ shows the hydrodynamic radius as a function of time. Using formulas (1-3) from that reference, I can convert the hydrodynamic radius, $R_{h}$, for aggregates into the number of monomers $n$ per aggregate:

$$
\begin{gathered}
R_{h}=\frac{L \sqrt{1-x^{2}}}{2 \ln \frac{1+\sqrt{1-x^{2}}}{x}} \\
x=\frac{d}{L}+\frac{.37 d(L-d)}{L^{2}} \\
n=\lambda L
\end{gathered}
$$

where $L$ is the length of an aggregate and $\lambda$ is the number of monomers per unit length. I set $\lambda$ equal to 1.6 as used in Lomakin et al., ${ }^{3}$ Kusumoto et al., ${ }^{101}$ and Tomski and Murphy. ${ }^{102}$ The other parameters are: $n$ is the number of monomers per aggregate, and $d$ is the diameter of an aggregate. I assume that all of the aggregates could be treated as 
cylinders, as in Yong et al. ${ }^{103}$ which says that micelles have a spherocylindrical shape. I set $d=8 \mathrm{~nm}$ for all the aggregates ${ }^{3,101}$ independent of $L$. I used Mathematica to invert equation (3.9) and find a value of $L$ for each $R_{h}$ in Lomakin et al. ${ }^{3}$ The $L$ was then used to calculate $n$ for each value of $R_{h}$.

I calculate a theoretical curve from my model for the time evolution of the number of monomers per aggregate and compare with the experimental results of Lomakin et al. ${ }^{3}$ and Kusumoto et al. ${ }^{101}$ The two experiments were similar, but were done at different temperatures and this may have a significant effect on $k_{n}$ but not $k_{e}{ }^{27}$ Though the steps in my model remain the same, differences in experimental conditions give different numerical values for the parameters in my model to fit each experiment. Differences in experimental conditions giving different numerical values for the parameters in my model is consistent with Lee ${ }^{104}$ in which it is reported that the average length of fibrils is sensitive to pressure and temperature. I define $\left[\beta_{N T}\right],\left[\beta_{T x}\right]$, and $\left[\beta_{M}\right]$ as the percentages of the total number of monomers in non-toxic aggregates (micelles), toxic aggregates (nuclei), and mature aggregates, respectively. Similarly, $m_{N T}$ and $m_{T x}$ are the average numbers of monomers in a micelle and nucleus, respectively. Literature supports a micelle having 25 monomers on average and a nucleus having an average of 10 monomers. ${ }^{2,3,99,27,103}$ I calculated the maximum number of monomers per aggregate, defined as parameter $m^{*}$, which varies depending on the experimental conditions. The

value of $m^{*}$ is determined from the long-time, asymptotic value of the experimental data. The parameters $a_{N T}, a_{T x}$, and $a_{M}$ are the total numbers of non-toxic aggregates, 
toxic aggregates, and mature aggregates, respectively, and $N$ is the total number of monomers in the entire system.

$$
\begin{array}{r}
{\left[\beta_{N T}\right]=\frac{m_{N T} \times a_{N T}}{N} \times 100 \%} \\
{\left[\beta_{T x}\right]=\frac{m_{T x} \times a_{T x}}{N} \times 100 \%} \\
{\left[\beta_{M}\right]=\frac{m^{*} \times a_{M}}{N} \times 100 \%} \\
n=\frac{\left[\beta_{N T}\right]+\left[\beta_{T x}\right]+\left[\beta_{M}\right]}{\left[\beta_{N T}\right]}+\frac{\left[\beta_{T x}\right]}{m_{N T}}+\frac{\left[\beta_{M}\right]}{m_{T x}^{*}}
\end{array}
$$

Using eq. (3.15) I was able to obtain good fits to both sets of experimental data. The fit in Fig. 3.5a for the data from Lomakin et al. ${ }^{3}$ was done with $\left[\beta_{N T}\right]$ initially set at $50 \%,\left[\beta_{T x}\right]$ initially set at $50 \%$, and $\left[\beta_{M}\right]=0$. The parameter $m^{*}$ used for this fit was set to 340. This fit showed significant deviation from the data for small times. I will do further work to achieve a better fit. A fit was produced for the Kusumoto data using $\left[\beta_{N T}\right],\left[\beta_{T x}\right]$, and $\left[\beta_{M}\right]$ all initially at $33.3 \%$ and $m^{*}$ of 600 . 

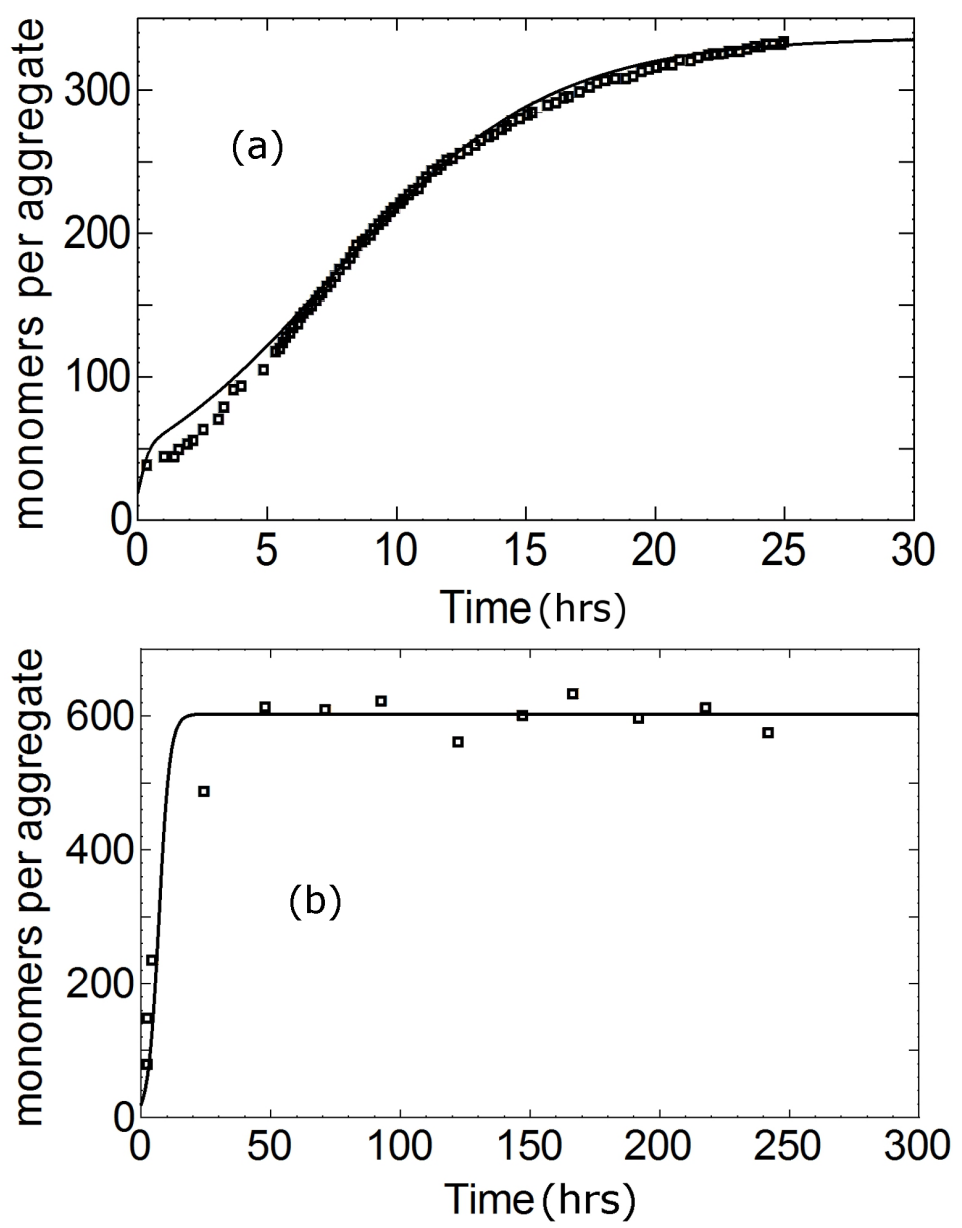

Fig. 3.5 Theoretical fits (-------) to experimental data ( $\square$ ) for the number of monomers $n$ per aggregate for two different experiments. (a) Lomakin et al., ${ }^{3}$ (b) Kusumoto et al. ${ }^{101}$

One thing that is gained from Figure 3.5 is that for large times a state of equilibrium is reached in which the average size of aggregates is stabilized. Another thing learned from Figure 3.5 is the approximate number of proteins in a mature $A \beta$ fibril. Since aggregates that are smaller than mature fibrils can be detected when it comes to getting the radius of hydration and some aggregates may never turn to mature fibrils, the average number of proteins in mature fibrils is likely to be larger than the asymptotic values in the two graphs in Figure 3.4. A question is why the asymptotic 
value for the Figure $3.4 \mathrm{~b}$, about 600 proteins, is approximately twice the asymptotic value of the Figure 3.4a. Both experiments were done at similar experimental conditions and by the same group. Slight variations in experimental preparation procedures such as purification and synthesis may significantly change the peptide system's fibrillogenic properties. A study showed that $A \beta$ fibrils are composed of five or six protofilaments, ${ }^{9}$ whereas another study showed $A \beta$ mature fibrils consist of two or three protofilaments. ${ }^{105}$ Experiments done by Pellarin showed mature fibrils having four protofilaments. ${ }^{106}$ 


\section{RESULTS OF MD COMPUTER SIMULATIONS FOR PROTOFIBRILLAR FORMATION OF CCß}

In this chapter I provide results of my MD computer simulations that provide molecular details of the dynamics of protofibrillar formation by the peptide cc $\beta$. I explain the steps that were taken to remove aphysical, computational artifacts so that the results would be physically valid. I also show how the MD results reinforce the idea that molecular heterogeneity is an important factor in understanding amyloid fibrillization.

\subsection{Computational Details}

The initial configuration of the 12 chain system in my REMD simulation consisted of four $\operatorname{cc} \beta$ coiled-coil $\alpha$-helix trimers arranged at the corners of a square, as shown in the first panel of Figure 4.1. Each trimer was created from the coordinates in the PDB 1S9Z file. The PDB file contains the atomic coordinates for a single $\operatorname{cc} \beta$ helix along with the translation and rotation coordinates for adding two additional $\operatorname{cc} \beta$ peptides to form a triplet. The four trimers at the corners of a square were separated by enough distance to ensure that there would be no van der Waals overlap between atoms from different trimers, with at least $24 \AA$ between the center of mass of one trimer and the center of mass of another trimer. The EEF1 force field with implicit solvent was used for modeling the solvation.

I performed the REMD simulation for 300 ns using 20 replicas with exponential spacing of the temperatures in a range of $380 \mathrm{~K}-600 \mathrm{~K}: 380.00 \mathrm{~K}, 389.25 \mathrm{~K}, 398.72 \mathrm{~K}$, $408.42 \mathrm{~K}, 418.36 \mathrm{~K}, 428.53 \mathrm{~K}, 438.96 \mathrm{~K}, 449.64 \mathrm{~K}, 460.58 \mathrm{~K}, 471.79 \mathrm{~K}, 483.27 \mathrm{~K}$, 
495.03K, 507.07K, 519.41K, 532.05K, 544.99K, 558.25K, 571.83K, 585.75K,

$600.00 \mathrm{~K}$. Each MD step was $2 \mathrm{fs}$ and I saved the configuration every 500 steps (making each frame 1ps apart). My temperature swap test was performed every 500 MD steps. I performed $20 \mathrm{ps}$ of REMD initialization and $20 \mathrm{ps}$ of REMD equilibration. The average acceptance rate for temperature swapping was $24 \%$, which is close to the ideal of $20 \% .^{107,108}$

In addition, I performed MD simulations at a constant temperature (CTMD). The parameters were the same in the CTMD as in the REMD simulations, except for the temperature. Also, in some of the CTMD simulations, there was a DROFF (confining potential radius) of $100 \AA$ as opposed to $600 \AA$ in the REMD simulations.

\subsection{Description of $\beta$-Sheet (Nucleus) Formation}

Figure 4.1 shows a sequence of frames taken at various times for a replica that formed two $\beta$-sheets, an important structural stage for the eventual fibrillization that could occur in a much larger system than I investigated. During the $300 \mathrm{~ns}$ covered in this simulation, this replica changed its temperature many times. 


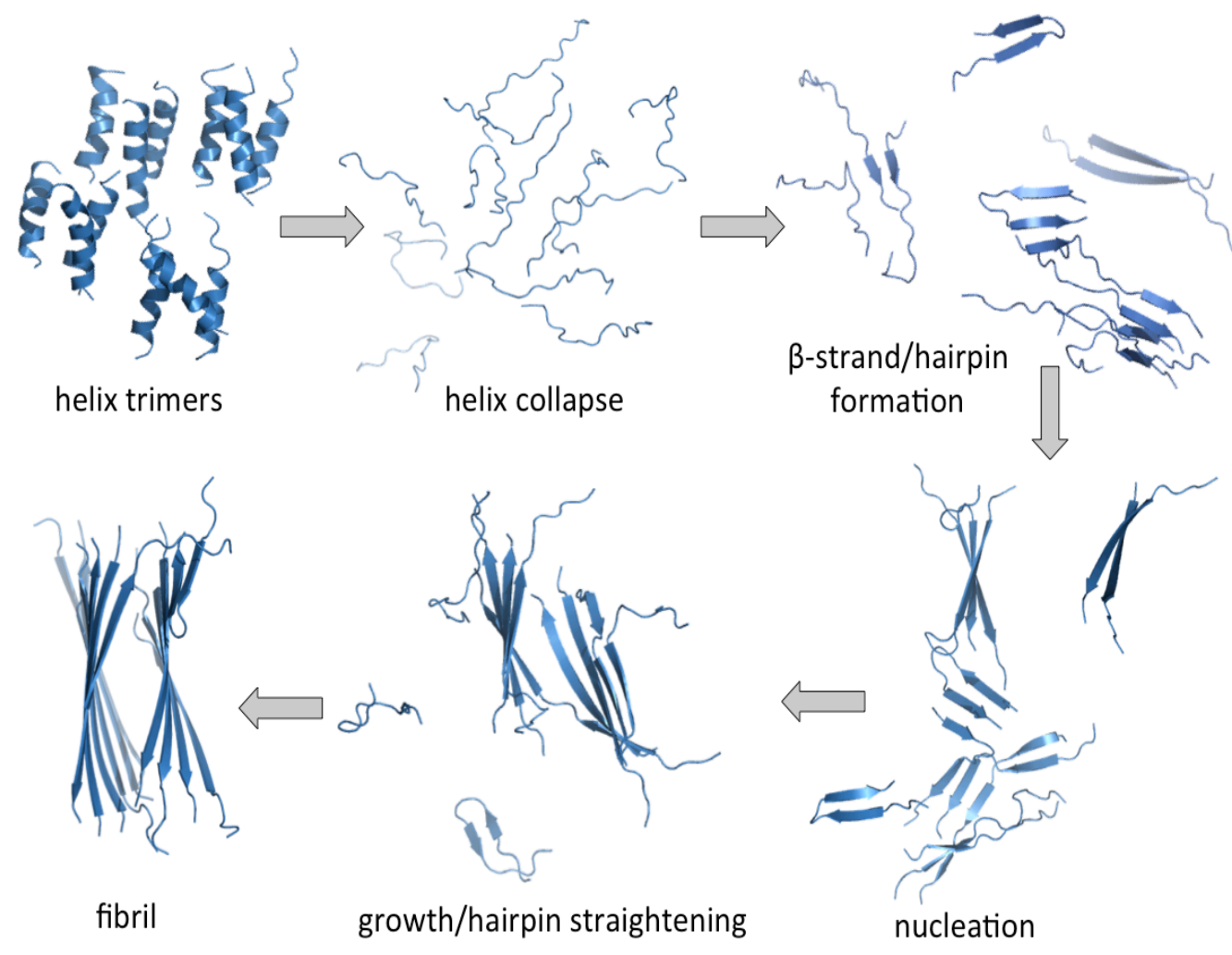

Fig. 4.1 Frames from the REMD simulations showing steps in the helix structural transition to $\beta$-strands and the formation of $\beta$-sheets.

My REMD simulation was performed in a temperature range from $380 \mathrm{~K}$ to $600 \mathrm{~K}$ in order to investigate $\beta$-chain formation and aggregation. These relatively high temperatures cause the initial $\alpha$-helical trimers to rapidly unfold so that the system quickly progresses to the random coil/ $\beta$-hairpin/ $\beta$-aggregate stages that I am interested in investigating. Experimental observations on $\operatorname{cc} \beta$ found that at $310 \mathrm{~K}$, the $\alpha$-helical configuration is unstable. ${ }^{42}$ Consistent with this, all of my replicas lost their initial $\alpha$ helical conformations within 1 ns. I focus attention on one replica, replica 3, which created the structure that most resembles a protofilament. The process of fibrillation for replica 3 is summarized in Figure 4.1. The last frame in Fig. 4.1 (from replica 3) looks 
like a protofilament steric zipper. It has similarities to the transthyretin single protein. ${ }^{109}$ For amyloids in general, some aggregates have parallel $\beta$-sheets, some have antiparallel $\beta$-sheets, ${ }^{110}$ and some have both parallel and antiparallel $\beta$-sheets. ${ }^{111,112}$

For the replicas that created a $\beta$-aggregate, after the proteins get out of the $\alpha$-helix secondary structure, some of the segments of the chain are in random coil and some are in $\beta$-structure. The proteins associate with each other in a disorganized aggregate, like a micelle. They then reorganize to form the $\beta$-aggregate.

To quantify the organization of the last frame of replica 3 (t-300 ns), which is shown in Figure 4.1, I calculated the nematic order parameter $S$ for the larger of the two $\beta$-sheets, which contains six $\operatorname{cc} \beta$ chains. The nematic order parameter ${ }^{113}$ is a measure of the alignment of rods in a system. I set $S$ to be the largest eigenvalue of the matrix

$$
Q_{\alpha \beta}=\frac{1}{N_{\text {protein }}} \sum_{j=1}^{N_{\text {protein }}}\left(\frac{3}{2} a_{j \alpha} a_{j \beta}-\frac{1}{2} \delta_{\alpha \beta}\right)
$$

where $j$ is an index that identifies each $\operatorname{rod}, \alpha$ and $\beta$ represent laboratory Cartesian coordinates, and $\overrightarrow{a_{\jmath}}$ is the unit direction vector for $\operatorname{rod} j$. In my $\operatorname{cc} \beta$ system, the protein chains are not perfectly rigid rods. I chose the direction vector, $\overrightarrow{a_{j}}$ for each protein to be the principal axis of the $\mathrm{C}_{\alpha}$ inertia tensor $I_{\alpha \beta}=\sum_{i=1}^{N_{a t o m s}} m_{i}\left(r_{i}^{2} \delta_{\alpha \beta}-r_{i \alpha} r_{i \beta}\right)$ corresponding to the smallest value of the moment of inertia for that chain. This provides a direction that is an average for the $\mathrm{C}_{\alpha}$ atoms in the chain. $r_{i}$ is the distance from the center of mass of the protein to each of the middle eleven $\mathrm{C}_{\alpha}$ atoms in the protein. I used only the middle eleven of the 17 residues because a $\beta$-strand protein, 
even when attached to other proteins, often has considerable flexibility for the amino acids near either end. Figure 4.2 shows for replica 3 the nematic order parameter as a function of time for the proteins that make up the largest $\beta$-sheet. A nematic order parameter of one means that there is perfect alignment of the protein direction vectors and zero means total isotropy in the arrangement of the protein direction vectors.

Characteristic structures for the six chains are also displayed at various times during the simulation. Since these results are from an REMD simulation, a single replica such as replica 3 changes temperature often. The temperature of replica 3 at any time is shown by the color of the curve. The order of colors from high temperature to low temperature is: red $\rightarrow$ ellow $\rightarrow$ green $\rightarrow$ cyan $\rightarrow$ blue.

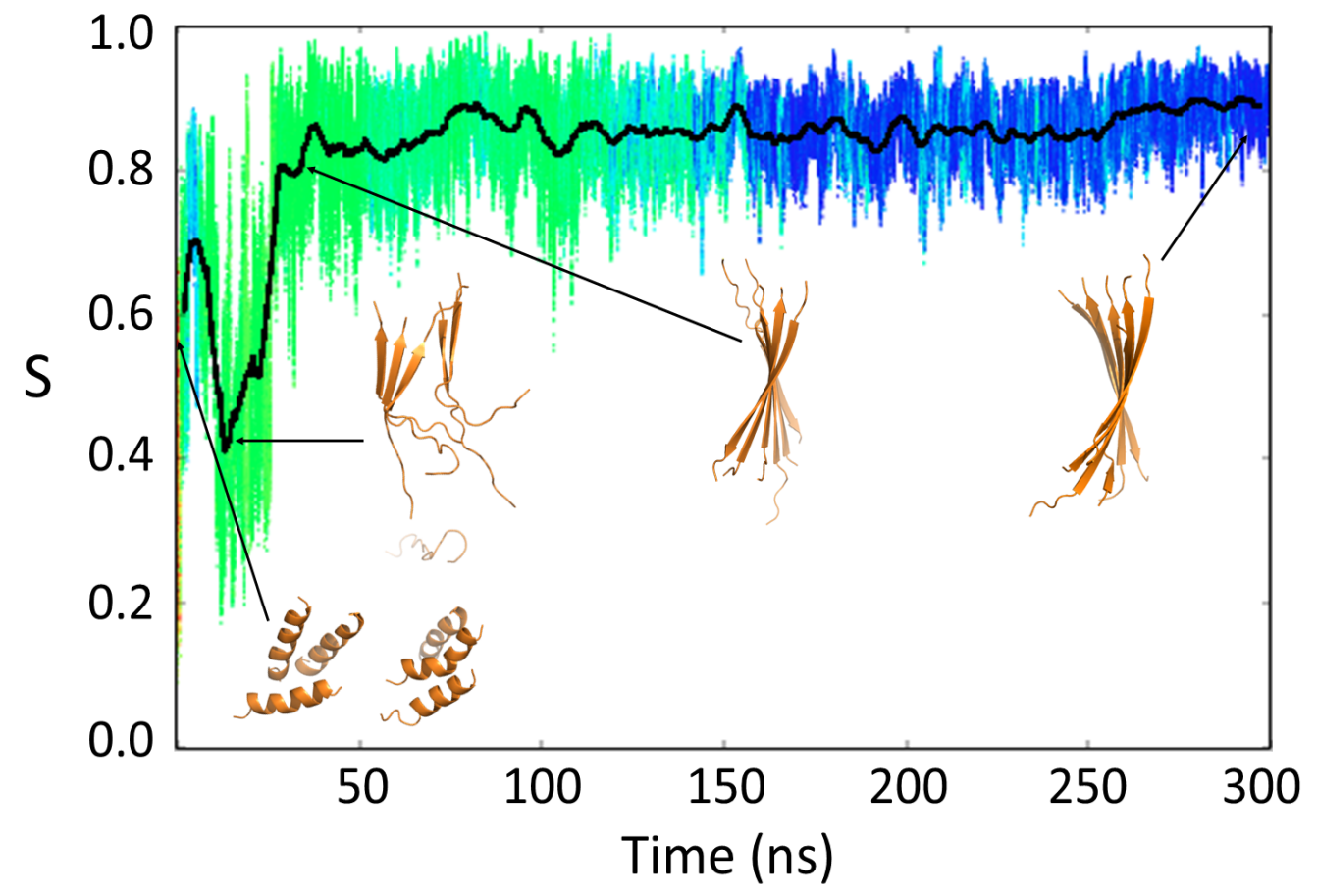

Figure 4.2 The nematic order parameter $S$ for the proteins that make up the largest $\beta$ sheet in replica 3. Characteristic structures for the six chains are displayed at various times during the simulation. The temperature of replica 3 at any time is shown by the color of the curve. The order of colors from high temperature to low temperature is: red $\rightarrow$ ellow $\rightarrow$ green $\rightarrow$ cyan $\rightarrow$ blue. 
Figure 4.2 displays large changes in $S$ during the first 40 ns while the six $\beta$ strands undergo major rearrangements relative to each other. By $50 \mathrm{~ns}$, the six chains have settled into a low energy, stable steric zipper $\beta$-sheet configuration with much smaller fluctuations in $S$. In the $\beta$-sheets formed in the simulations, each $\beta$-strand has a twist. The average twist angle for the larger of the two $\beta$-sheets for the last $200 \mathrm{~ns}$ is $10^{\circ}$. This is a similar twist angle to that calculated in Knowles et al. ${ }^{114}$

\subsection{Phase Transition}

Experimental observations ${ }^{42}$ find that at a temperature of $277 \mathrm{~K}, \operatorname{cc} \beta$ exists as a stable coiled-coil $\alpha$-helix trimer, whereas at $310 \mathrm{~K}$ the helical structure converts to $\beta$ strands. REMD investigations on a single trimer by Strodel et al. ${ }^{40}$ found that a $\beta$-sheet composed of three $\operatorname{cc} \beta$ chains is stable up to a temperature of $440 \mathrm{~K}$.

In order to investigate the thermodynamics of the process, I calculated various parameters from my REMD simulations. Figure 4.3 displays the heat capacity as a function of temperature for my system of 12 chains. The heat capacity at each temperature was calculated from the fluctuations in the energy using equation (4.2)

$$
C_{v}=\frac{\overline{E^{2}}-\bar{E}^{2}}{R T^{2}}
$$

I found that for all temperatures, the system had settled into its final configuration, whether ordered into an aggregate or not, by $100 \mathrm{~ns}$. Therefore, I calculated the 
equilibrium $C_{V}$ at all temperatures using the final $200 \mathrm{~ns}$ of the simulations, which covers the period from $100 \mathrm{~ns}$ to $300 \mathrm{~ns}$.

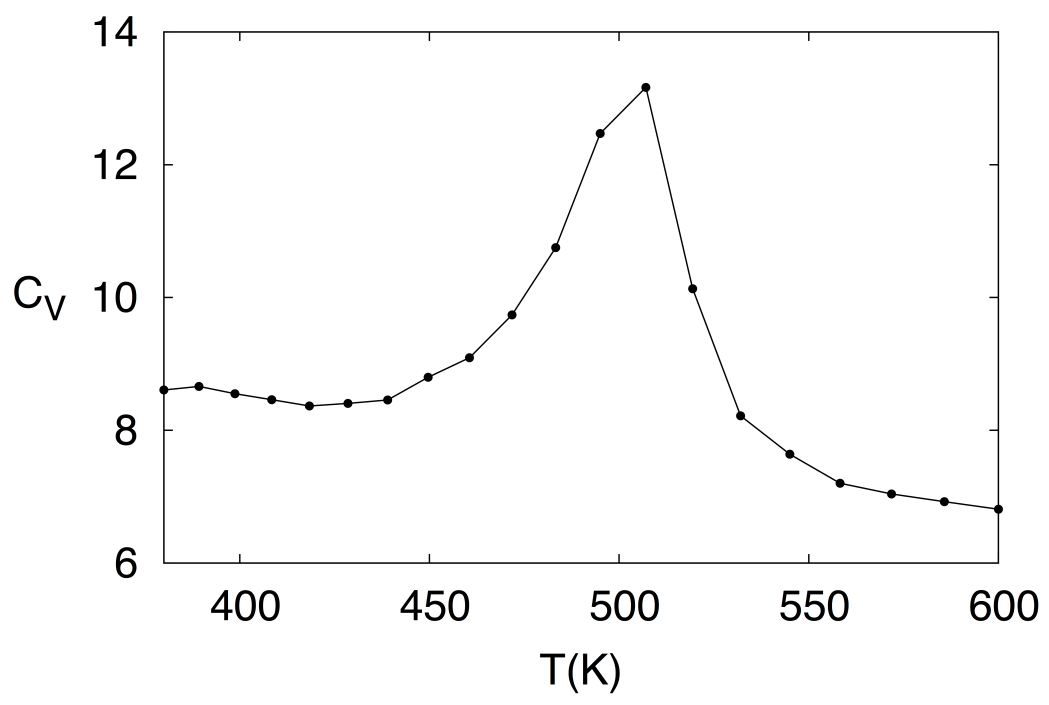

Figure 4.3 The heat capacity of my 12 chain system as a function of temperature.

The process in my system fits the thermodynamic characteristics of a phase transition, as can be seen by the peak in the heat capacity curve at the temperature point $507 \mathrm{~K}$. In order to obtain more information on the dynamics that produce this heat capacity peak, I calculated additional parameters. The criterion to determine if two chains are a part of the same aggregate is if the center of mass of any residue of one chain is within a certain distance of the center of mass of any residue of the other chain. This distance is $1 / 2\left(d^{r e s 1}+d^{r e s 2}\right)$, in which $d^{\text {res }}=3.5 \AA+2 *\left|\boldsymbol{R}_{C \alpha}-\boldsymbol{R}_{C O M}\right| . \boldsymbol{R}_{C \alpha}$ and $\boldsymbol{R}_{C O M}$ are the positions of the Carbon $\alpha$ atom and the center of mass of the residue respectively. The reason for the $3.5 \AA$ in the formula for $d^{\text {res }}$ is if the Carbon $\alpha$ atom and the center of 
mass of the residue have the same position. $3.5 \AA$ is the distance between two covalently bonded Carbon atoms. Figure 4.4 displays the number of chains in the largest aggregate as a function of temperature, averaged over the last $200 \mathrm{~ns}$. It is clear from Figure 4.4 that significant aggregation occurs only for temperatures below 500K.

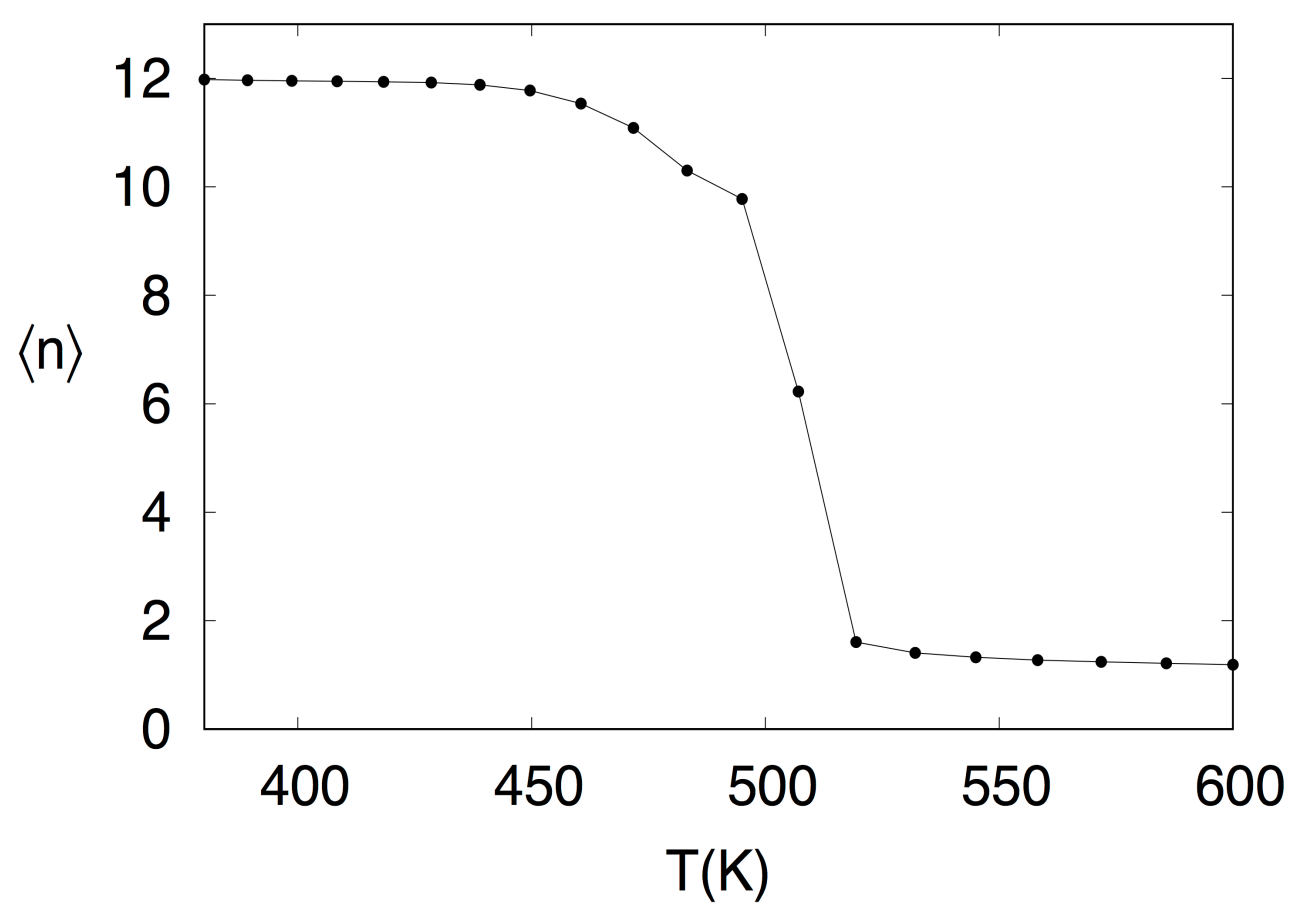

Figure 4.4 The number of chains in the largest aggregate as a function of temperature, averaged over the last $200 \mathrm{~ns}$.

\subsection{Validity of Results}

I examined the two $\beta$-sheet structure formed in replica 3. This type of structure may be important in allowing $\operatorname{cc} \beta$ to form amyloid-like protofilaments. As can be seen in Figure 4.5a, the two $\beta$-sheets are held together by a variety of sidechain interactions. 
The blue (positively charged arginine) and red (negatively charged glutamic acid) sidechains are oppositely charged sidechains which provide electrostatic forces between the $\beta$ sheets. Figure $4.5 \mathrm{~b}$ shows that similar sidechains extend out on both sides of each sheet, providing the ability for additional sheets to be added to increase the size of the $\beta$ aggregate. Each sheet also contains white spheres representing hydrophobic sidechains (isoleucine and leucine) on each side, both in the middle between the two sheets (Figure 4.5a) and on the outside (Figure 4.5b). The specific location of these hydrophobic regions may provide specificity that directs the proper alignment ${ }^{115}$ of sheets as they are added to the aggregate.

I found that $\operatorname{cc} \beta$ formed $\beta$-sheets that are held together by electrostatic forces between charged sidechains. Charged sidechains are located on both sides of each $\beta$ sheet, providing the opportunity for additional $\beta$-sheets to be added in layers to increase the size of the aggregate. Regions of hydrophobic sidechains may provide specificity that facilitates alignment of charged sidechains as the $\beta$-aggregate grows in size 

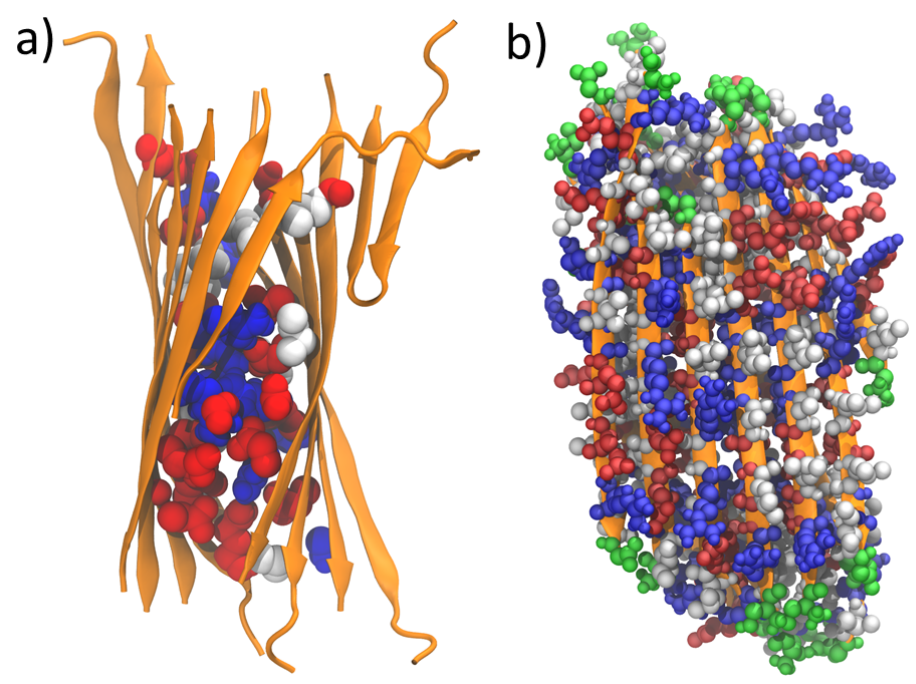

Figure 4.5 From replica 3, positively charged (blue) and negatively charged (red) sidechains provide strong binding interactions: (a) between two $\beta$-sheets to hold the sheets together (b) on the outside of the two $\beta$-sheets to allow the addition of more $\beta$ sheets to the aggregate. Hydrophobic sidechains (white spheres) may provide specificity to facilitate alignment.

In addition to determining the average number of chains in the largest aggregate, I also investigated the amount of order or stability in the size of the largest aggregate. In Figure 4.6 I plot the normalized number fluctuations $\chi_{\mathrm{n}}$ for the chains in the largest aggregate

$$
\chi_{n}=\frac{<n^{2}>-<n>^{2}}{<n>^{2}}
$$




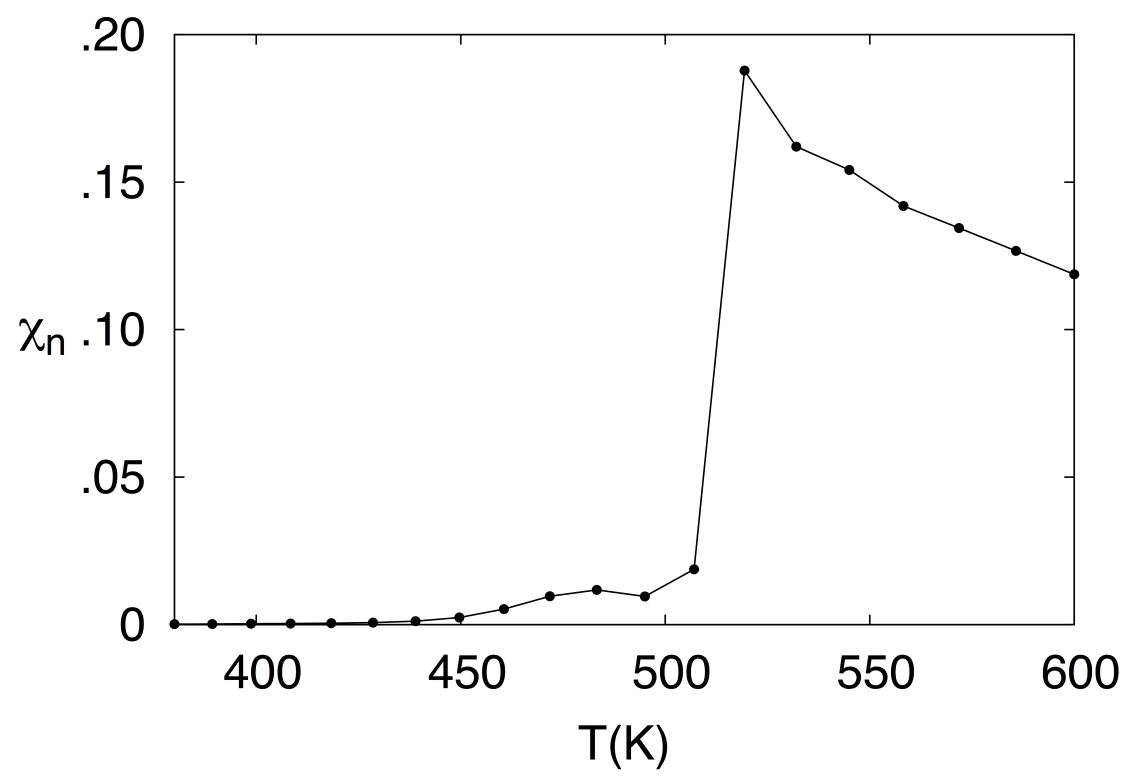

Figure 4.6 Stability of the largest aggregate based upon a parameter $\chi_{\mathrm{n}}$ that measures the fluctuations in the number of chains in the largest aggregate.

Even though there are a much larger number of chains in the largest aggregate for temperatures below $500 \mathrm{~K}$ compared to temperatures above $500 \mathrm{~K}$, Fig. 4.6 shows that below $500 \mathrm{~K}$ the structure of the system is much more stable with very small fluctuations. The isothermal compressibility can be calculated using the expression ${ }^{35}$ $\kappa_{T}=\chi_{n}(V / K T)$. Figure 4.6 for $\chi_{\mathrm{n}}$ shows that the structurally organized aggregate below $500 \mathrm{~K}$ has a significantly smaller $\kappa_{\mathrm{T}}$ compared to the disorganized system above $500 \mathrm{~K}$, which makes physical sense. Experimenal determination of $\kappa_{\mathrm{T}}$ is a possibilty for validating my results. 


\subsection{Temperature Window of $\beta$-Sheet Formation}

The figures shown above reflect properties of the multichain system and interactions between the chains. I also investigated the behavior and dynamics of individual chains within the system.

Hydrogen bond rearrangement is an important factor accompanying the structural transition from $\alpha$ helix to $\beta$ sheets. At all of the temperatures that I used in the simulations, the $\alpha$ helices fall apart too quickly ( $<1 \mathrm{~ns})$ for me to monitor them and their intra helix hydrogen bonds. However, I was able to monitor the formation of interchain hydrogen bonds as $\beta$ strands form and aggregate into $\beta$ sheets. Figure 4.7 shows the time development of interchain hydrogen bonds for different temperatures. Since these results are from REMD simulations, the characteristic times are not reliable. However, the plots show that below $507 \mathrm{~K}$, the number of interchain hydrogen bonds increases as the replicas form a $\beta$ sheet, whereas above $507 \mathrm{~K}$ the number of interchain hydrogen bonds never stabilizes at a high number, implying that a $\beta$ sheet never forms. 

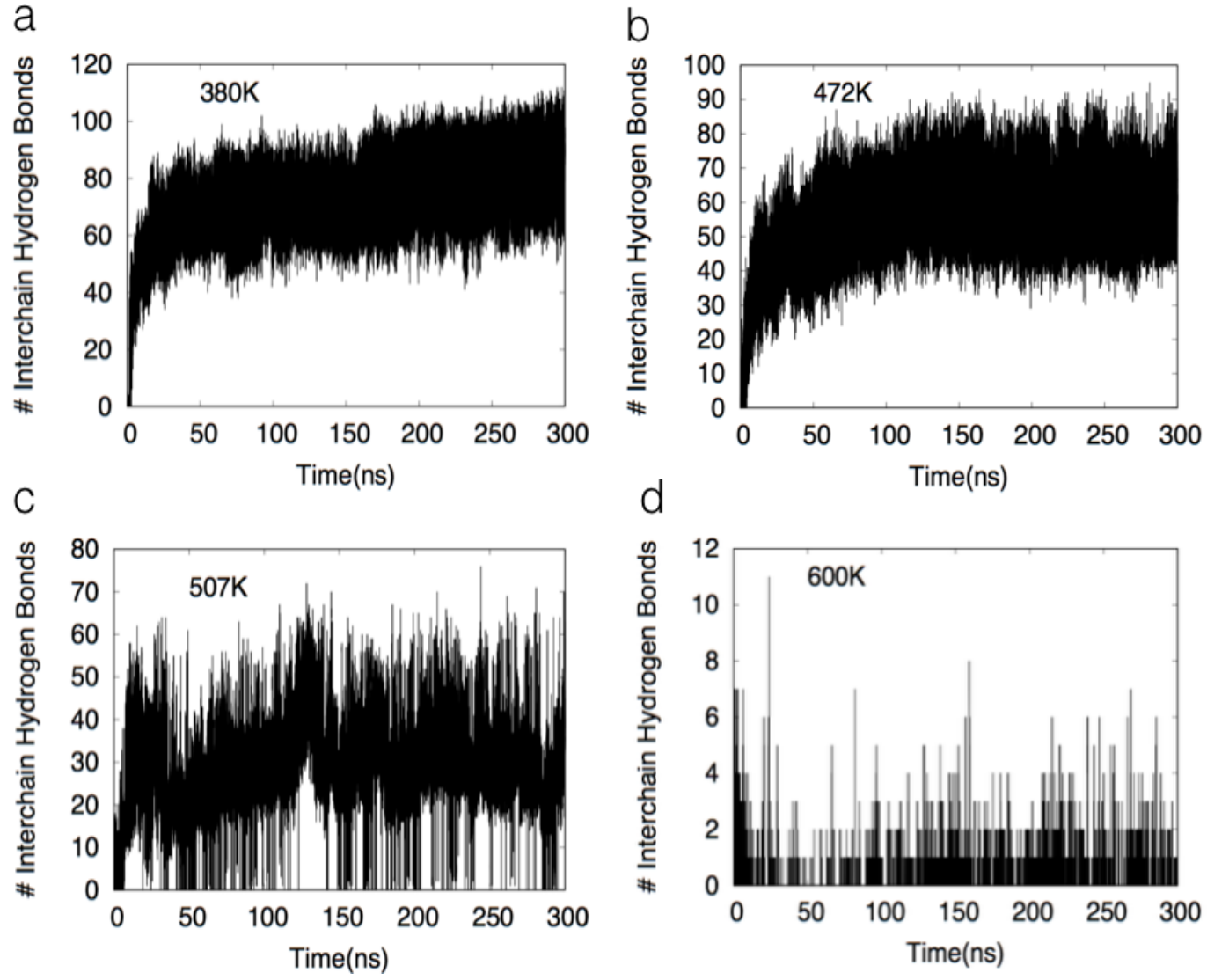

Fig 4.7 Number of interchain hydrogen bonds versus time. (a) $380 \mathrm{~K}$ (b) $472 \mathrm{~K}$ (c) $507 \mathrm{~K}$ (d) $600 \mathrm{~K}$.

In order to better understand the role of hydrogen bonds in the structural transition, I examined how the number of hydrogen bonds varied depended on temperature at the end of my simulations. Figure 4.8 displays the number of interchain hydrogen bonds, as well as the number of intrachain hydrogen bonds, averaged over the final $200 \mathrm{~ns}$. It can be seen that the number of interchain hydrogen bonds drops suddenly to almost zero above $500 \mathrm{~K}$, which matches the temperature dependence displayed in Figure 4.4 for the number of chains in the largest aggregate. In contrast, the number of intrachain bonds begins to drop at a temperature of $450 \mathrm{~K}$, a lower 
temperature than the dramatic drop that occurs for the interchain hydrogen bonds at $500 \mathrm{~K}$.

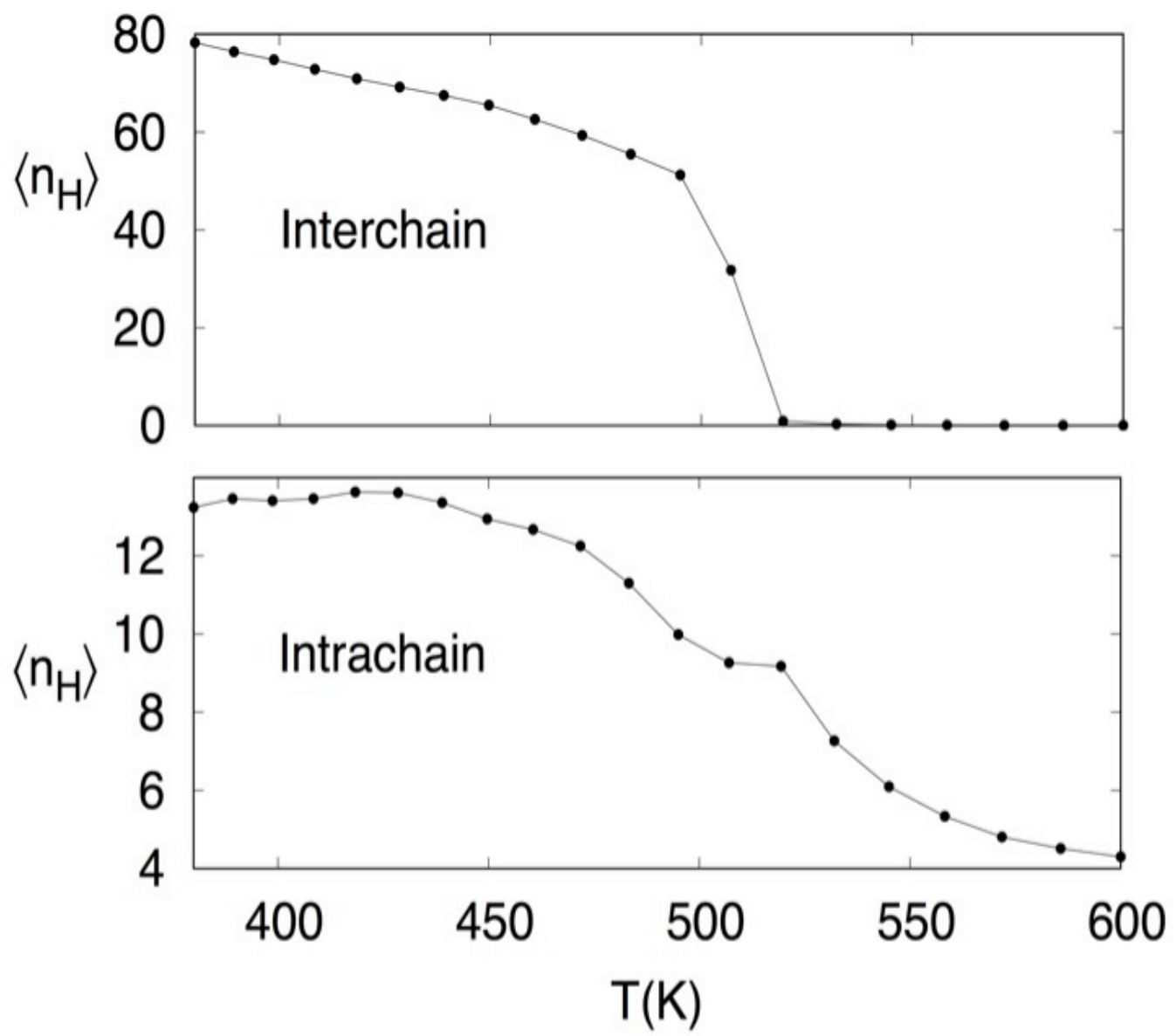

Figure 4.8 Average number of hydrogen bonds as a function of temperature. Top: interchain hydrogen bonds, Bottom: intrachain hydrogen bonds. 


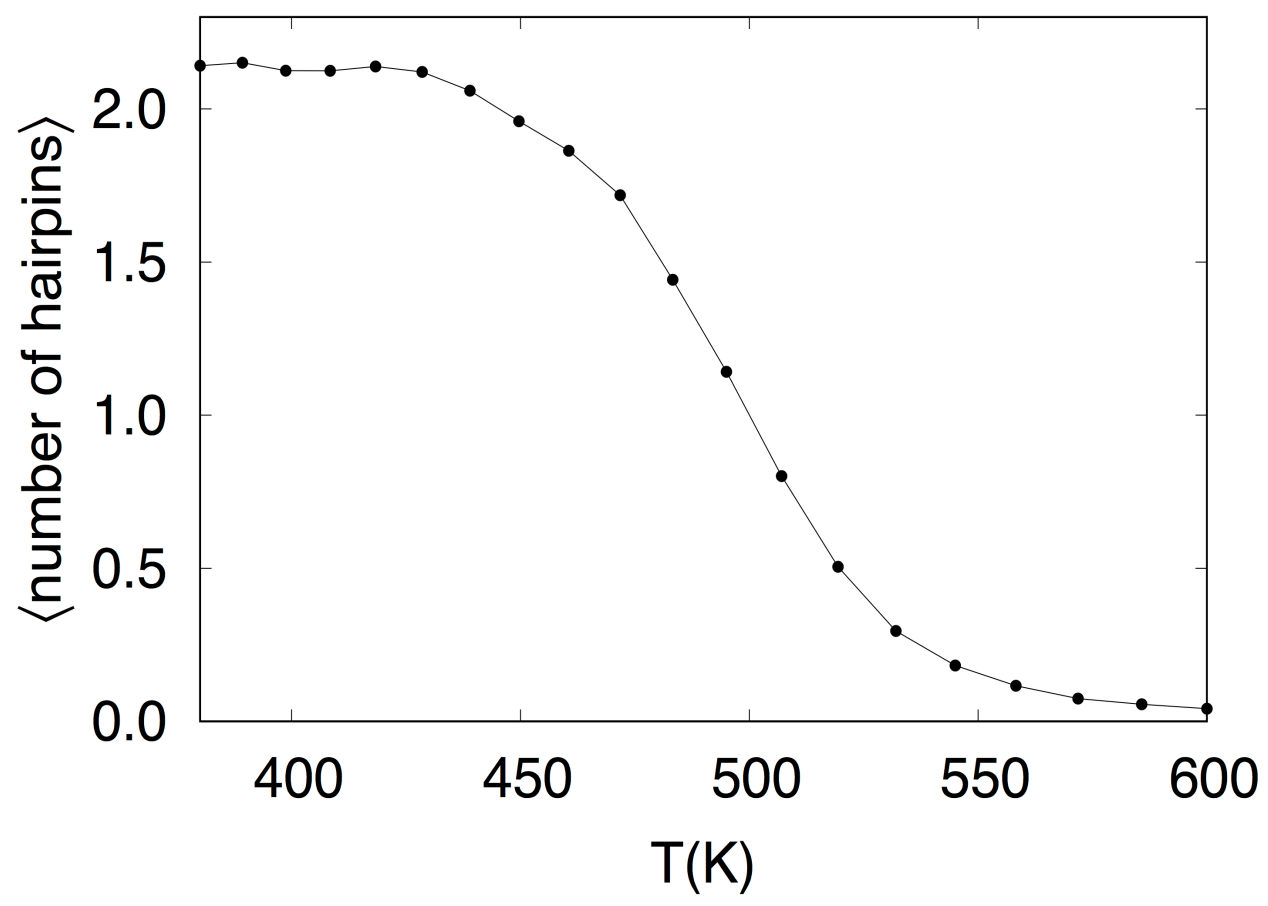

Figure 4.9 The average number of chains that are in a hairpin structure as a function of temperature.

The graphs above that reflect the behavior of the multichain system show a clear structural transition involving $\beta$-structure that occurs around 500K (Figure 4.3). Above $500 \mathrm{~K}$, the system is highly disordered with negligible aggregation (Figure 4.4) and large fluctuations (Figure 4.6). Below 500K there is almost complete aggregation of all $\beta$-chains into stable aggregates with little fluctuation.

In my REMD simulation, if the system does not spend significant time above $450 \mathrm{~K}$, the individual chains remain in $\beta$-hairpin conformation and form non-amyloid structures. However, if the system spends significant time above 450K, Figure 4.9 
shows that the probability for an individual chain to be in a hairpin structure drops significantly. This allows individual chains to straighten-out into $\beta$-strands, a process that is facilitated by hairpin structures forming hydrogen bonds with other chains. The straightened $\beta$-strands are in a conformation that allows them to bind into $\beta$-sheets and form protofibrils. However, Figure 4.8 shows that above 500K, the probability for forming the interchain hydrogen bonds necessary to form $\beta$-sheets drops dramatically. As shown in Figure 4.4, this prevents the formation of aggregates above $500 \mathrm{~K}$.

The CTMD simulations showed that there tends to be hairpins at low temperatures and straightened-out chains at high temperatures.

To better understand the relevance of interchain and intrachain hydrogen bonds on the structure of individual protein chains, I determined the average number of chains that are in a hairpin structure as a function of temperature. This is plotted in Figure 4.9. A chain was counted as being in a hairpin configuration if it contained four or more intrachain hydrogen bonds. The drop in Figure 4.9 above $450 \mathrm{~K}$ closely matches the temperature dependence of the number of intrachain bonds displayed in Figure 4.8.

In general for amyloid proteins, the intermediate aggregates are composed of $\beta$ strands in the hairpin state along with $\beta$-strands that are extended. The smaller oligomers tend to have a higher percentage of $\beta$-strands in the hairpin state than the mature fibrils. Hairpins seem more likely to straighten out as an aggregate gets $\operatorname{bigger}^{26}$ because there are more interactions between chains that can dominate over the intrachain hydrophobic interactions that stabilize the hairpin conformation of a chain. However, even in large $\beta$-sheets, proteins in a hairpin conformation can serve as the growing end of the sheet. $26,22,116,117$ 
Two different structural transition processes involving hydrogen bonds are required for aggregation into fibrils: the breaking of intrachain hydrogen bonds to allow $\beta$-hairpin proteins to straighten, and the subsequent formation of interchain hydrogen bonds during $\beta$ aggregation into amyloid fibrils. For my REMD investigations, I found that the temperature dependence of these two different $\beta$ structural transition processes results in the existence of a temperature window that the protein experiences during the process of forming protofibrillar structures. Appearance of the temperature window allows me to investigate the dynamics on a molecular level. If the protein system in my simulations spends all of its time at either lower temperatures or higher temperatures relative to this window, fibrillization will not occur. 


\subsection{Free Energy and Heterogeneity}

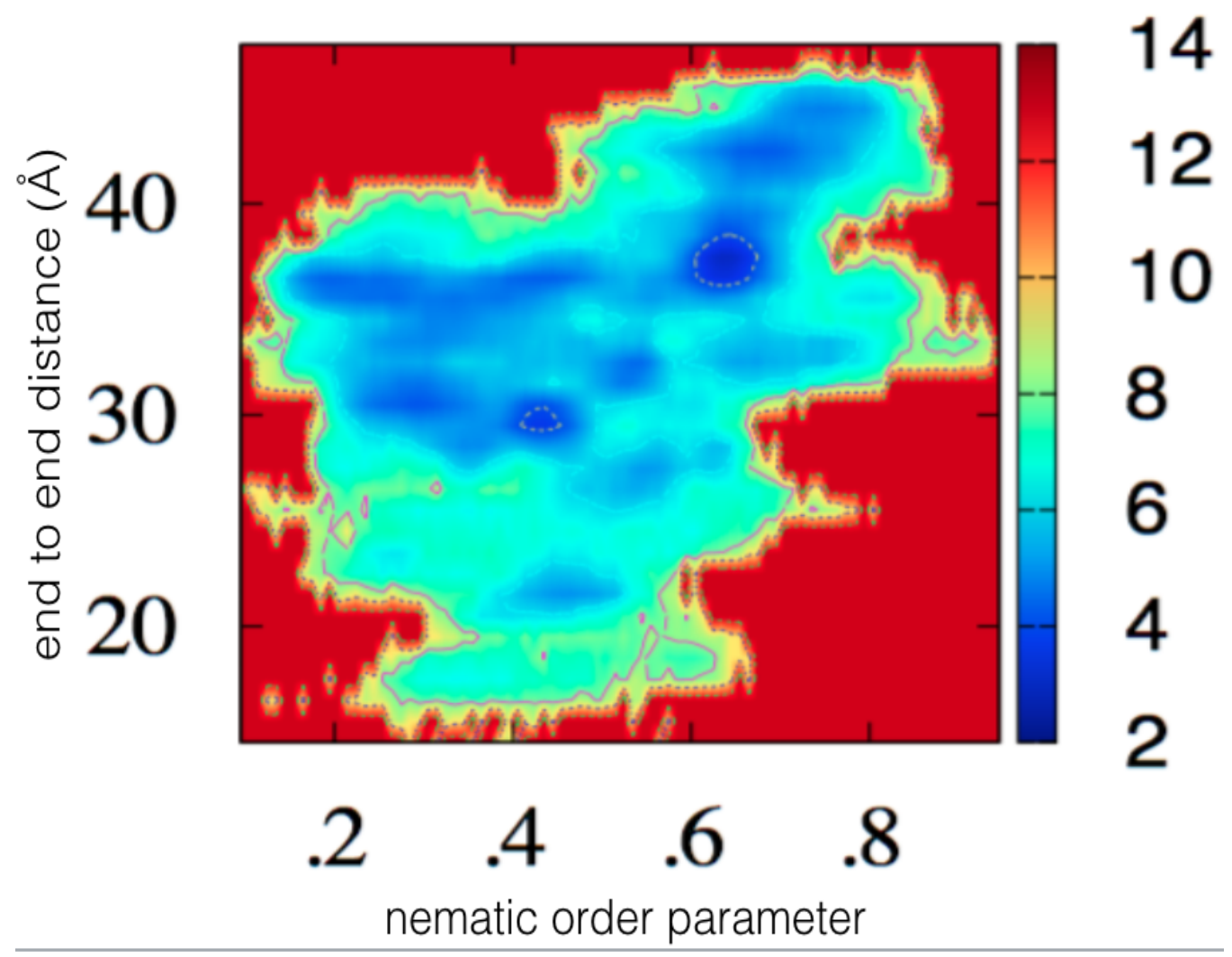

Figure 4.10 Free energy at $380 \mathrm{~K}$ for all $300 \mathrm{~ns}$ (minus $20 \mathrm{ps}$ from initialization and 20 ps from equilibration). The end to end distance $\left(\mathrm{d}_{\mathrm{ee}}\right)$ is the distance, averaged over all 12 proteins, from the $\mathrm{C}_{\alpha}$ at one end to the $\mathrm{C}_{\alpha}$ at the other end of the protein.

The free energy for $380 \mathrm{~K}$ for the last $300 \mathrm{~ns}$ (minus $20 \mathrm{ps}$ from initialization and 20 ps from equilibration) is shown in Figure 4.10. The reason I included the free energy for the entire simulation is to include the structures going from a disordered aggregate to an ordered $\beta$-aggregate. The free energy was calculated by first creating bins. Each bin included a range of nematic order parameter values and a range of $d_{e e}$ values. For each bin, the number of frames within the $S$ and $d_{e e}$ ranges of that bin for a certain temperature (for whatever replica at the frame that fits the criteria) was counted. The 
formula for the free energy, $G=-R T \ln (P)$, was used, with $P$ the fraction of frames that are within a certain bin.

The graph in Figure 4.10 shows that there is a lot of heterogeneity for the earlier structures as well as for the $\beta$-aggregates that form later in the simulation. There are many places of dark blue, and each dark blue island can have many significantly different structures. The structure of the system is not defined totally by $d_{e e}$ and $S$.

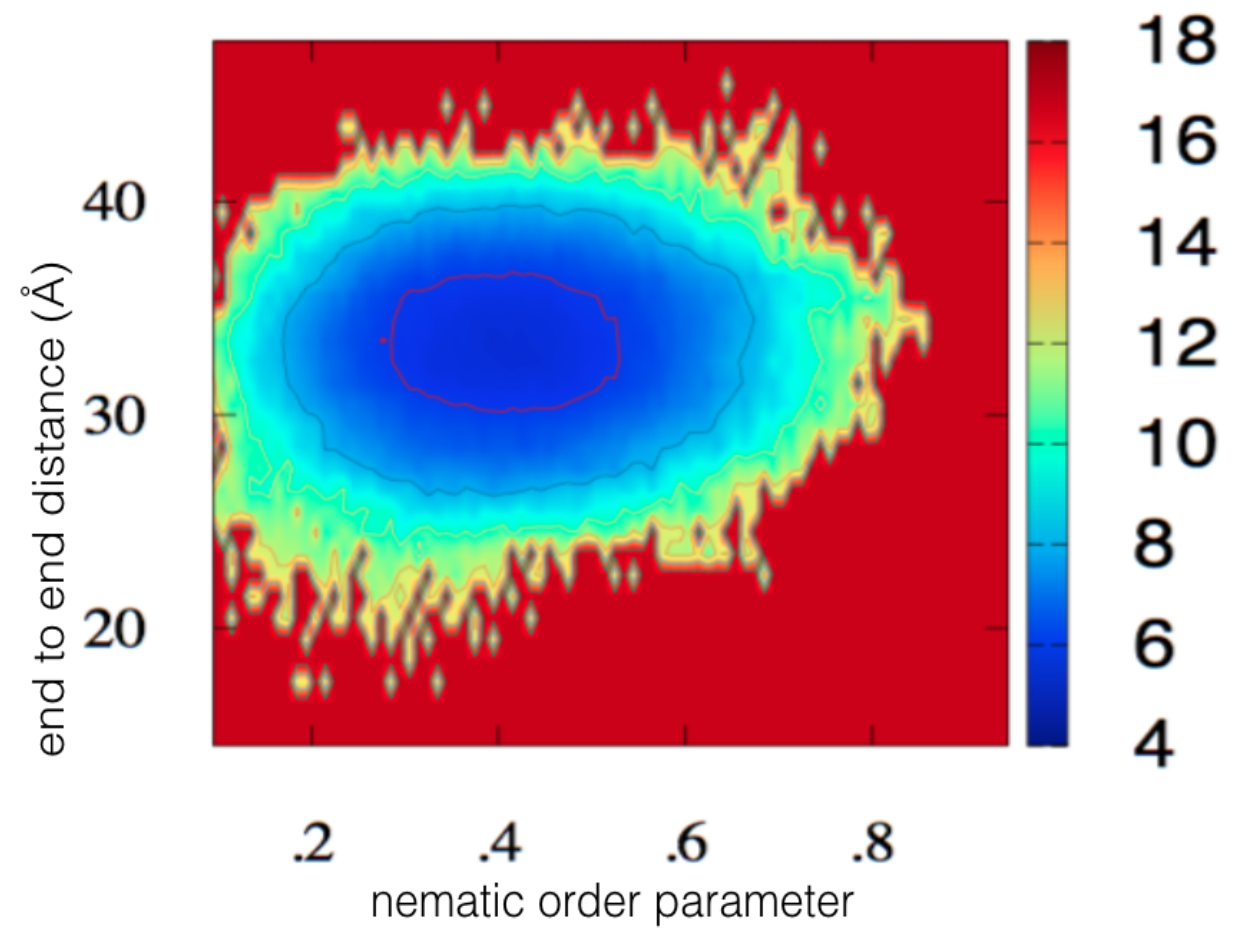

Figure 4.11 Free energy at $507 \mathrm{~K}$ as a function of $d_{e e}$ and $S$ for the last $200 \mathrm{~ns}$.

The free energy in Figure 4.11 is at the temperature 507K. (The transition temperature 500K was not one of the specific temperatures in my REMD simulation.) There is only one free energy well. If this was a first-order transition, there would likely be two free energy wells at the transition temperature. According to polymer theory, a first-order phase transition happens for a homogenous polymerizing system. ${ }^{118}$ 
However, a glass-like transition happens for a polymerizing system that is heterogenous. ${ }^{118}$ A glass-like transition is a continuous transition. ${ }^{118}$ It has similarities to a second-order phase transition, but is not strictly a second-order phase transition. ${ }^{118}$

The last frame of replica 3 is shown as the last panel of in Figure 4.1. The last frame in replica 3 looks more like a steric protofilament than the last frame of any of the other replicas. Replicas 14 and 16 also look like steric zipper protofilaments. However, you can see large structural differences among replica 3, 14, and 16. Figure 4.12 shows the last frame of replicas 3,14 , and 16 next to each other.
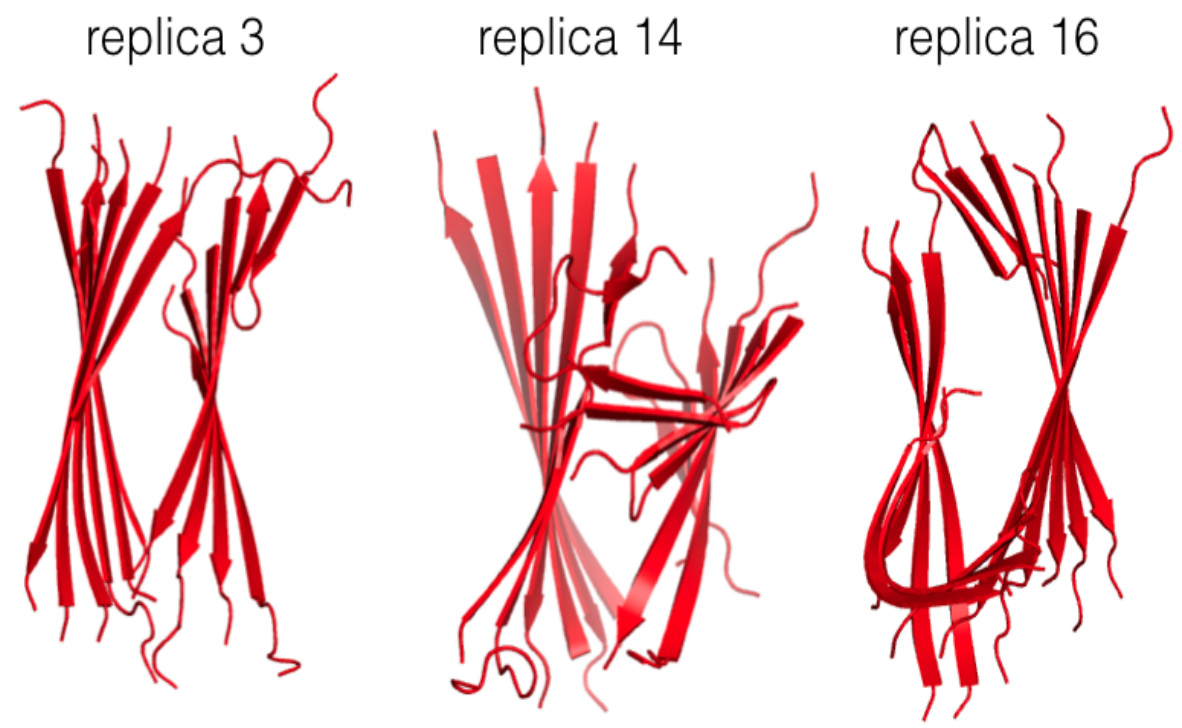

Figure 4.12 Last frame of replicas 3, 14, and 16 next to each other.

\subsection{Protein to Protein Interactions}

The CTMD showed that the fibril formation occurs much quicker with a smaller confining potential radius as opposed to a larger confining potential radius. With a smaller confining potential radius, there is more protein to protein contact and hence more chance for proteins to catalyze each other to change other proteins' secondary 
structure. Increasing the concentration in amyloid structures has the effect of speeding up the aggregation process. 


\section{CONCLUSIONS}

To investigate the kinetics and thermodynamics of amyloid formation in amyloidogenic proteins, I used various mathematical and computational tools. Specifically, I developed a mathematical model to investigate the population kinetics of structural conversions leading to $\mathrm{A} \beta$ fibril formation. I then used molecular dynamics (MD) computational simulations to investigate the molecular details of the structural transformations and calculate thermodynamic parameters for amyloid formation. I employed a special form of molecular dynamics, Replica Exchange Molecular Dynamics (REMD), which is described in section 2.2 .5 , to simulate a system of $12 \operatorname{cc} \beta$ proteins. I found that the results of both the mathematical modeling of the kinetics and the molecular dynamics computational simulations could be explained in terms of two important molecular-level aspects. Section 5.1 emphasizes how hydrogen bond rearrangements correlate to aggregation phase transitions and population dynamics of secondary structure. In section 5.2 I describe how my results show the prevalence of the heterogeneity in the systems I studied. Section 5.3 discusses more ideas for MD simulations and suggests experimental ideas based on my results.

\subsection{Hydrogen Bonds}

The model I developed to fit the population of secondary structure kinetics data that was available from experiments is described in Fig. 3.1 and Table 3.1. For my fitting of the Kirkitadze data ${ }^{28}$, Fig. 3.2, I fit changes of secondary structure with time. Changes in secondary structure with time correlate with changes in the number of 
interchain and intrachain hydrogen bonds. $\alpha$ helices have a lot of intrachain hydrogen bonds, whereas $\beta$-sheets have a lot of interchain hydrogen bonds.

Two different structural transition processes involving hydrogen bonds are required for aggregation into fibrils: the breaking of intrachain hydrogen bonds to allow hairpin proteins to straighten, and the subsequent formation of interchain hydrogen bonds during $\beta$ aggregation into amyloid fibrils. For my REMD investigations, I found that the temperature dependence of these two different $\beta$ structural transition processes results in the existence of a temperature window that my system experiences during the process of forming protofibrillar structures. If a replica in my simulation spends all of its time at either lower temperatures or higher temperatures relative to the temperature window, fibrillization will not occur.

My MD simulations look at molecular-level details of some of the stages whose transition kinetics I mathematically modeled. A summary of the steps from the MD simulations of the $\operatorname{cc} \beta$ replica that created the aggregate that most resembled a steric zipper protofilament is shown in Figure 4.1.

In Figure 4.7, the number of interchain hydrogen bonds versus time for four different temperatures is displayed. For the temperatures $380 \mathrm{~K}$ and $472 \mathrm{~K}$, the number of interchain bonds increases as time advances. The reason for the increase in the number of interchain hydrogen bonds is the formation of $\beta$-sheets. For temperature $507 \mathrm{~K}$, the number of interchain hydrogen bonds fluctuates rapidly. The reason for the rapid fluctuation is that this is the transition temperature, in which some replicas form $\beta$-sheets and some don't. At $600 \mathrm{~K}$, there are few interchain hydrogen bonds throughout the simulation, since the temperature is too high to allow $\beta$-sheets. 
I found that the hydrogen bond rearrangement is an important factor in the structural transition and helps elucidate the temperature dependency. The initial configuration of the 12 chains was all $\alpha$-helix, which means many intrachain hydrogen bonds. At all temperatures, the $\alpha$ helices fell apart too quickly ( $<1 \mathrm{~ns})$ for me to be able to monitor the helical intrachain hydrogen bonds. I was able to monitor the increase of interchain hydrogen bonds as $\beta$ sheets form, as shown in Figure 4.7. Since my simulations used REMD, the time scales in Fig. 4.7 are not reliable, but the temperature dependencies are. I examined the temperature dependencies more carefully. Figure 4.8 displays the number of interchain hydrogen bonds, as well as the number of intrachain hydrogen bonds, averaged over the final $200 \mathrm{~ns}$. It can be seen that the number of interchain hydrogen bonds drops suddenly to almost zero above $500 \mathrm{~K}$, which matches the temperature dependence displayed in Figure 4.4 for the number of chains in the largest aggregate. In contrast, the number of intrachain hydrogen bonds begins to drop at a temperature of $450 \mathrm{~K}$, a lower temperature than the dramatic drop that occurs for the interchain hydrogen bonds at $500 \mathrm{~K}$.

At high temperature the system being entropically favored makes it so that random coils are the preferred secondary structure. Hydrogen bonds don't last long in proteins in random coil because each protein can sample many different configurations. At low temperature the system being enthalpically favored makes the proteins have many hydrogen bonds. The proteins having many hydrogen bonds lowers the energy, but also makes it a low entropy configuration.

The graphs that reflect the behavior of the multichain system show a clear structural transition involving $\beta$-structure that occurs around 500K (Figure 4.3). Above 
$500 \mathrm{~K}$, the preferred configuration of the system is highly disordered with negligible aggregation (Figure 4.4) and large fluctuations (Figure 4.6). Below 500K there is almost complete aggregation of all $\beta$-chains into stable aggregates with little fluctuation.

Interestingly, my REMD simulation displayed dynamics defined by a temperature window for the formation of organized $\beta$-sheets in the region of $450 \mathrm{~K}$ to $500 \mathrm{~K}$. If the system does not spend significant time above $450 \mathrm{~K}$, the individual chains remain in hairpin conformation and form non-amyloid structures. If the system spends significant time above $450 \mathrm{~K}$, Figure 4.9 shows that the probability for an individual chain to be in a hairpin structure drops significantly. This allows individual chains to straighten-out into $\beta$-strands, a process that is facilitated by hairpin structures forming hydrogen bonds with other chains. The straightened $\beta$-strands are in a conformation that allows them to form interchain hydrogen bonds and organize into $\beta$-sheets that can form protofibrils. However, Fig. 4.8 shows that above 500K, the probability for forming the interchain hydrogen bonds necessary to form $\beta$-sheets drops dramatically. As shown in Fig. 4.4, this prevents the formation of any type of aggregate above $500 \mathrm{~K}$.

To better understand the relevance of interchain and intrachain hydrogen bonds on the structure of individual protein chains, I investigated more closely the hairpin intermediate conformation that prevents chains from organizing into $\beta$-sheets. I determined the average number of chains that are individually in a hairpin structure as a function of temperature. This is plotted in Fig. 4.9. A chain was counted as being in a hairpin configuration if it contained four or more intrachain hydrogen bonds. The drop 
in Fig. 4.9 above $450 \mathrm{~K}$ closely matches the temperature dependence of the number of intrachain bonds displayed in Fig. 4.8.

\subsection{Heterogeneity}

The cc $\beta$ protein system I simulated had 12 chains, each with 17 amino acid residues. Based on simulations performed by other researchers of two short $A \beta(16-22)$ peptides that showed significant heterogeneity in forming dimers ${ }^{18}$ we can expect significantly more heterogeneity in my system of $\operatorname{cc} \beta$ aggregation. The reasons for the greater heterogeneity are that my system has more chains and each $\operatorname{cc} \beta$ chain is longer than each $A \beta(16-22)$ peptide. We can expect even more heterogeneity in systems of Kirkitadze et al. ${ }^{28}$ and Walsh et al. ${ }^{37}$, having a large number of $A \beta(1-40)$ proteins.

The mathematical model I used to fit population dynamics of secondary structure is shown in Figure 3.1. To represent the heterogeneity in the system, in the mathemaitcal model I used sub-compartments which are mathematically equivalent to heterogeneous distributions. My fit to the kinetic data in the article by Kirkitadze ${ }^{28}$, Fig 3.2, had four sub-compartments which shows a significant amount of heterogeneity. I spent a lot of time working to get the parameters just right for the best fit, including the number of sub-compartments. The more sub-compartments, the more heterogeneity. ${ }^{77}$ The shapes in the population dynamics curves are significantly different with four subcompartments than with no sub-compartments, as shown in Figure 3.15 of in the book Modeling Infectious Diseases in Humans and Animlas by Keeling. ${ }^{77}$ My fitting to the data from the Walsh article ${ }^{37}$, Fig 3.3a, had six sub-compartments, displaying even more heterogeneity than in the data from the Kirkitadze article. ${ }^{28}$ 
In addition to the heterogeneity in my modeling of the kinetics, my MD simulations also manifested significant heterogeneity. From the MD results, I calculated the free energy of various configurations of the 12 chain system. The amount of heterogeneity in my $\operatorname{cc} \beta$ simulations can be seen in the free energy plot for a temperature of $380 \mathrm{~K}$, Figure 4.10. There are a many different regions on the free energy plot that have low free energy. In addition, each region of low free energy on that diagram contains different structures.

According to polymer theory, a first-order phase transition can occur in a homogenous polymerizing system. ${ }^{118}$ However, a glass-like transition happens for a polymerizing system that is heterogenous. ${ }^{118}$ A glass-like transition is a continuous transition. ${ }^{118}$ It has similarities to a second-order phase transition, but is not strictly a second-order phase transition. ${ }^{118}$ There weren't enough data points in the heat capacity curve, Figure 4.3, to decide whether it was a first-order phase transition. However, I deduced from the free energy diagram at the transition temperature, 507K, Figure 4.11 that my system did not display a first-order phase-transition. There is only one free energy well that spans the different large-scale configurations, showing a continuous phase transition.

The heterogeneity in my REMD simulations was also shown by the different replicas. Not all replicas formed $\beta$-aggregates. Those replicas that produced $\beta$ aggregates all produced a different $\beta$-aggregate or $\beta$-aggregates. The last frame of the three replicas that most resembled steric zipper protofilaments are shown in Figure 4.12. They look significantly different from each other. 
Figure 4.2 shows the nematic order parameter $S$ for the proteins that make up the largest $\beta$-sheet for the replica that most resembled a steric zipper protofilament. A value of one for $S$ means there is perfect alignment of all the protein direction vectors and zero means total isotropy in the protein direction vectors. Figure 4.2 displays large changes in $S$ during the first 40 ns while the six $\beta$-strands undergo major rearrangements relative to each other. This shows a lot of heterogeneity in the early micelle-like aggregates.

\subsection{Directions for Future Work}

If I had more time, I would have wanted to do simulated annealing constant temperature MD to help verify the temperature window hypothesized from my REMD simulation. The procedure for simulated annealing constant temperature MD is as follows: (1) perform constant temperature MD with the system of $12 \operatorname{cc} \beta$ proteins at $600 \mathrm{~K}$ for $15 \mathrm{~ns}$; (2) take the last frame of the simulation at $600 \mathrm{~K}$ and use it for another constant temperature simulation at $590 \mathrm{~K}$ for $15 \mathrm{~ns}$; (3) take the last frame of the simulation at $590 \mathrm{~K}$ and use it for the first frame of a simulation at $580 \mathrm{~K}$; (4) continue this process down to $380 \mathrm{~K}$.

Other simulations of cc $\beta$ would be around 20 different 150 ns constant temperature MD simulations of my $\operatorname{cc} \beta$ system at the same temperature and other conditions. As mentioned in Chapter 4, $100 \mathrm{~ns}$ is about the time it takes a system to equilibrate. Because MD has some random numbers in it, each simulation is likely to create unique structures and dynamics for each simulation, even though they all would 
have the same conditions. Seeing how different each simulation is from the other ones will help to understand how heterogenous the aggregation process is.

Both KFFEAAAKKFFE and KFFEYNGKKFFE have four amno acids flanked on both sides by the de novo peptide KFFE. KFFEAAAKKFFE does a better job of forming amyloid fibrils than KFFEYNGKKFFE. ${ }^{119}$ KFFEYNGKKFFE folds into a stable $\beta$-hairpin. ${ }^{119}$ MD simulations on a system of several KFFEYNGKKFFE peptides and on a system of several KFFEAAAKKFFE peptides could help investigate why KFFEYNGKKFFE has a higher propensity than KFFEAAAKKFFE to form $\beta$-hairpins.

For $\operatorname{cc} \beta$, temperature is the control parameter for its structural transitions. One idea for experiments on $\operatorname{cc} \beta$ is to have the experiment start with high temperature and slowly lower the temperature. This change in the temperature during the experiment can help facilitate the process of fibrillization.

For the REMD simulations on $\operatorname{cc} \beta$, I found hydrogen bonds between backbone atoms. This made my results generalizable to any protein because every protein has the same backbone. The two different structural transitions involving hydrogen bonds that are controlled by temperature in $\operatorname{cc} \beta$ may also create a window for other amyloid forming proteins, but the window-defining control parameter may not be temperature. For other amyloid forming proteins in which temperature is not the structural transition control parameter, perhaps another control parameter may be used based on the knowledge gained from the current work on $\operatorname{cc} \beta$ for which there is a temperature window.

Figure 3.4 helps explain the energetics of how Trifluoroethanol (TFE) affects the secondary structure. Intermolecular bonds are formed at low TFE concentrations, 
whereas, at high TFE concentrations, intramolecular hydrogen bonds are preferred. ${ }^{85}$ The temperature window found for $\operatorname{cc} \beta$ can help explain why too much TFE for $A \beta$ mentioned in section 3.4.1 impedes fibrillization. A condition is necessary in which there can be breaking of intrachain hydrogen bonds, not only for the $\alpha$ helices to break once they've formed but also for the hairpins to straighten out.

One idea is to experimentally start out with a high concentration of TFE for A $\beta$. This can stabilize the $\alpha$ helices. Then the experimentalist can lower the concentration of TFE after many $\alpha$ helices have been formed. Lowering the concentration of TFE helps not only the $\alpha$ helices to break but also helps straighten out the $\beta$-strands.

An idea for therapies to prevent amyloid diseases is to stabilize the proteins in their hairpin state. One idea for a control parameter to be put into medications are affibodies. ${ }^{120,121}$ Affibodies are binding proteins derived from the $\mathrm{Z}$ domain of staphylococcal protein A. ${ }^{121}$ Affibodies are able to stabilize the hairpin state of proteins by clamping down on them. ${ }^{122}$

\section{REFERENCES}

1. Schnabel, J., Protein folding: The dark side of proteins. Nature 2010, 464 (7290), $828-9$.

2. Lomakin, A.; Teplow, D. B.; Kirschner, D. A.; Benedek, G. B., Kinetic theory of fibrillogenesis of amyloid beta-protein. Proc Natl Acad Sci US A 1997, 94 (15), 79427.

3. Lomakin, A.; Chung, D. S.; Benedek, G. B.; Kirschner, D. A.; Teplow, D. B., On the nucleation and growth of amyloid beta-protein fibrils: detection of nuclei and quantitation of rate constants. Proc Natl Acad Sci US A 1996, 93 (3), 1125-9. 
4. Stefani, M.; Dobson, C. M., Protein aggregation and aggregate toxicity: new insights into protein folding, misfolding diseases and biological evolution. J Mol Med 2003, 81 (11), 678-99.

5. Keshet, B.; Yang, I. H.; Good, T. A., Can size alone explain some of the differences in toxicity between beta-amyloid oligomers and fibrils? Biotechnol Bioeng 2010, 106 (2), 333-7.

6. Mousseau, N.; Derreumaux, P., Exploring the early steps of amyloid peptide aggregation by computers. Acc Chem Res 2005, 38 (11), 885-91.

7. Pallitto, M. M.; Murphy, R. M., A mathematical model of the kinetics of betaamyloid fibril growth from the denatured state. Biophys $J$ 2001, 81 (3), 1805-22.

8. Zerovnik, E., Amyloid-fibril formation. Proposed mechanisms and relevance to conformational disease. Eur J Biochem 2002, 269 (14), 3362-71.

9. Sipe, J. D., Amyloid proteins : the beta sheet conformation and disease. WileyVCH: Weinheim, 2005.

10. Kelly, J. W.; Balch, W. E., Amyloid as a natural product. J Cell Biol 2003, 161 (3), 461-2.

11. Otzen, D. E., Amyloid fibrils and prefibrillar aggregates : molecular and biological properties. Wiley-VCH: Weinheim, Germany, 2013; p xxviii, 481 p.

12. Giurleo, J. T.; He, X.; Talaga, D. S., Beta-lactoglobulin assembles into amyloid through sequential aggregated intermediates. J Mol Biol 2008, 381 (5), 1332-48.

13. Hellstrand, E.; Boland, B.; Walsh, D. M.; Linse, S., Amyloid beta-protein aggregation produces highly reproducible kinetic data and occurs by a two-phase process. ACS Chem Neurosci 2010, 1 (1), 13-8.

14. Jarrett, J. T.; Berger, E. P.; Lansbury, P. T., Jr., The carboxy terminus of the beta amyloid protein is critical for the seeding of amyloid formation: implications for the pathogenesis of Alzheimer's disease. Biochemistry 1993, 32 (18), 4693-7.

15. Xue, W. F.; Homans, S. W.; Radford, S. E., Systematic analysis of nucleationdependent polymerization reveals new insights into the mechanism of amyloid selfassembly. Proc Natl Acad Sci U S A 2008, 105 (26), 8926-31.

16. Rahimi, F.; Bitan, G.; SpringerLink (Online service), Non-fibrillar Amyloidogenic Protein Assemblies - Common Cytotoxins Underlying Degenerative Diseases. Springer Netherlands,: Dordrecht, 2012; p. 1 online resource. http://dx.doi.org/10.1007/978-94-007-2774-8. 
17. Dobson, C. M., Protein misfolding, evolution and disease. Trends Biochem Sci 1999, 24 (9), 329-32.

18. Santini, S.; Wei, G.; Mousseau, N.; Derreumaux, P., Pathway complexity of Alzheimer's beta-amyloid Abeta16-22 peptide assembly. Structure 2004, 12 (7), 124555 .

19. Hurshman, A. R.; White, J. T.; Powers, E. T.; Kelly, J. W., Transthyretin aggregation under partially denaturing conditions is a downhill polymerization. Biochemistry 2004, 43 (23), 7365-81.

20. Steckmann, T.; Awan, Z.; Gerstman, B. S.; Chapagain, P. P., Kinetics of peptide secondary structure conversion during amyloid beta-protein fibrillogenesis. $J$ Theor Biol 2012, 301, 95-102.

21. Cheon, M.; Chang, I.; Mohanty, S.; Luheshi, L. M.; Dobson, C. M.; Vendruscolo, M.; Favrin, G., Structural reorganisation and potential toxicity of oligomeric species formed during the assembly of amyloid fibrils. PLoS Comput Biol 2007, 3 (9), 1727-38.

22. Matthes, D.; Gapsys, V.; Daebel, V.; de Groot, B. L., Mapping the Conformational Dynamics and Pathways of Spontaneous Steric Zipper Peptide Oligomerization. PLoS ONE 2011, 6 (5), e19129.

23. Nelson, R.; Eisenberg, D., Structural models of amyloid-like fibrils. Adv Protein Chem 2006, 73, 235-82.

24. Eisenberg, D.; Nelson, R.; Sawaya, M. R.; Balbirnie, M.; Sambashivan, S.; Ivanova, M. I.; Madsen, A. O.; Riekel, C., The structural biology of protein aggregation diseases: Fundamental questions and some answers. Acc Chem Res 2006, 39 (9), 56875 .

25. Zheng, J.; Jang, H.; Ma, B.; Tsai, C. J.; Nussinov, R., Modeling the Alzheimer Abeta17-42 fibril architecture: tight intermolecular sheet-sheet association and intramolecular hydrated cavities. Biophys $J$ 2007, 93 (9), 3046-57.

26. Zhou, R., Molecular modeling at the atomic scale : methods and applications in quantitative biology. CRC Press, Taylor \& Francis Group: Boca Raton, 2015; p xix, 360 pages.

27. Sabate, R.; Gallardo, M.; Estelrich, J., Temperature dependence of the nucleation constant rate in beta amyloid fibrillogenesis. Int J Biol Macromol 2005, 35 (1-2), 9-13. 
28. Kirkitadze, M. D.; Condron, M. M.; Teplow, D. B., Identification and characterization of key kinetic intermediates in amyloid beta-protein fibrillogenesis. $J$ Mol Biol 2001, 312 (5), 1103-1119.

29. Ramirez-Alvarado, M.; Kelly, J. W.; Dobson, C. M., Protein misfolding diseases : current and emerging principles and therapies. Wiley: Hoboken, N.J., 2010; p xxx, $1045 \mathrm{p}$.

30. Serpell, L. C., Alzheimer's amyloid fibrils: structure and assembly. Biochim Biophys Acta 2000, 1502 (1), 16-30.

31. Petkova, A. T.; Buntkowsky, G.; Dyda, F.; Leapman, R. D.; Yau, W. M.; Tycko, R., Solid state NMR reveals a $\mathrm{pH}$-dependent antiparallel beta-sheet registry in fibrils formed by a beta-amyloid peptide. J Mol Biol 2004, 335 (1), 247-60.

32. Roher, A. E.; Baudry, J.; Chaney, M. O.; Kuo, Y. M.; Stine, W. B.; Emmerling, M. R., Oligomerizaiton and fibril asssembly of the amyloid-beta protein. Biochim Biophys Acta 2000, 1502 (1), 31-43.

33. Chimon, S.; Shaibat, M. A.; Jones, C. R.; Calero, D. C.; Aizezi, B.; Ishii, Y., Evidence of fibril-like beta-sheet structures in a neurotoxic amyloid intermediate of Alzheimer's beta-amyloid. Nat Struct Mol Biol 2007, 14 (12), 1157-64.

34. Murphy, R. M.; Pallitto, M. M., Probing the kinetics of beta-amyloid selfassociation. J Struct Biol 2000, 130 (2-3), 109-22.

35. Abney, J. R.; Scalettar, B. A.; Hackenbrock, C. R., On the measurement of particle number and mobility in nonideal solutions by fluorescence correlation spectroscopy. Biophysical Journal 1990, 58 (1), 261-265.

36. Fezoui, Y.; Teplow, D. B., Kinetic studies of amyloid beta-protein fibril assembly. Differential effects of alpha-helix stabilization. J Biol Chem 2002, 277 (40), 36948-54.

37. Walsh, D. M.; Hartley, D. M.; Kusumoto, Y.; Fezoui, Y.; Condron, M. M.; Lomakin, A.; Benedek, G. B.; Selkoe, D. J.; Teplow, D. B., Amyloid beta-protein fibrillogenesis. Structure and biological activity of protofibrillar intermediates. $J$ Biol Chem 1999, 274 (36), 25945-52.

38. Ding, F.; LaRocque, J. J.; Dokholyan, N. V., Direct observation of protein folding, aggregation, and a prion-like conformational conversion. J Biol Chem 2005, $280(48), 40235-40$. 
39. Steinmetz, M. O.; García-Echeverría, C.; Kammerer, R. A., Design of a CoiledCoil-based Model Peptide System toExplore the Fundamentals of Amyloid Fibril Formation. International Journal of Peptide Research and Therapeutics 2005, 11 (1), 43-52.

40. Strodel, B.; Fitzpatrick, A. W.; Vendruscolo, M.; Dobson, C. M.; Wales, D. J., Characterizing the first steps of amyloid formation for the ccbeta peptide. J Phys Chem B 2008, 112 (32), 9998-10004.

41. Missimer, J. H.; Steinmetz, M. O.; Baron, R.; Winkler, F. K.; Kammerer, R. A.; Daura, X.; van Gunsteren, W. F., Configurational entropy elucidates the role of saltbridge networks in protein thermostability. Protein Sci 2007, 16 (7), 1349-59.

42. Kammerer, R. A.; Kostrewa, D.; Zurdo, J.; Detken, A.; Garcia-Echeverria, C.; Green, J. D.; Muller, S. A.; Meier, B. H.; Winkler, F. K.; Dobson, C. M.; Steinmetz, M. O., Exploring amyloid formation by a de novo design. Proc Natl Acad Sci US A 2004, 101 (13), 4435-40.

43. Kammerer, R. A.; Steinmetz, M. O., De novo design of a two-stranded coiled-coil switch peptide. J Struct Biol 2006, 155 (2), 146-53.

44. Nölting, B.; SpringerLink (Online service), Protein Folding Kinetics Biophysical Methods. Second Edition. ed; Springer-Verlag Berlin Heidelberg,: Berlin, Heidelberg, 2006. http://dx.doi.org/10.1007/b138868.

45. Newman, M. E. J., Computational physics. Rev. and expanded [ed.] ed.; Createspace: Charleston, SC?, 2013; p xi, 549 p.

46. Landau, R. H.; Páez Mejía, M. J.; Bordeianu, C. C., Computational physics : problem solving with computers. 2nd revised and enlarged edition. ed.; Wiley-VCH ; John Wiley distributor: Weinheim Chichester, 2007; p xxi, 593 p.

47. Schlick, T.; SpringerLink (Online service), Molecular Modeling and Simulation: An Interdisciplinary Guide An Interdisciplinary Guide. In Interdisciplinary Applied Mathematics, [Online] Second. ed.; Springer Science+Business Media, LLC,: New York, NY, 2010; p. 1 online resource. http://dx.doi.org/10.1007/978-1-4419-6351-2.

48. Becker, O. M., Computational biochemistry and biophysics. M. Dekker: New York, 2001; p xii, 512 p. 
49. Brooks, B. R.; Brooks, C. L., 3rd; Mackerell, A. D., Jr.; Nilsson, L.; Petrella, R. J.; Roux, B.; Won, Y.; Archontis, G.; Bartels, C.; Boresch, S.; Caflisch, A.; Caves, L.; Cui, Q.; Dinner, A. R.; Feig, M.; Fischer, S.; Gao, J.; Hodoscek, M.; Im, W.; Kuczera, K.; Lazaridis, T.; Ma, J.; Ovchinnikov, V.; Paci, E.; Pastor, R. W.; Post, C. B.; Pu, J. Z.; Schaefer, M.; Tidor, B.; Venable, R. M.; Woodcock, H. L.; Wu, X.; Yang, W.; York, D. M.; Karplus, M., CHARMM: the biomolecular simulation program. J Comput Chem 2009, 30 (10), 1545-614.

50. Finkelstein, A. V.; Ptitsyn, O. Bebrary Inc., Protein physics a course of lectures. In Soft condensed matter, complex fluids and biomaterials series [Online] Academic Press,: Amsterdam ; Boston, 2002; pp. xix, 354 p. http://site.ebrary.com/lib/yale/Doc?id=10186414.

51. Frenkel, D.; Smit, B., Understanding molecular simulation : from algorithms to applications. 2nd ed.; Academic Press: San Diego, Calif. London, 2002; p xxii, 638 p.

52. Rao, F.; Caflisch, A., Replica exchange molecular dynamics simulations of reversible folding. J Chem Phys 2003, 119 (7), 4035-4042.

53. Sugita, Y.; Okamoto, Y., Replica-exchange molecular dynamics method for protein folding. Chemical Physics Letters 1999, 314 (1-2), 141-151.

54. Gc, J. B.; Bhandari, Y. R.; Gerstman, B. S.; Chapagain, P. P., Molecular dynamics investigations of the alpha-helix to beta-barrel conformational transformation in the RfaH transcription factor. J Phys Chem B 2014, 118 (19), 5101-8.

55. Pu, M.; Garrahan, J. P.; Hirst, J. D., Comparison of implicit solvent models and force fields in molecular dynamics simulations of the PB1 domain. Chemical Physics Letters 2011, 515 (4-6), 283-289.

56. Ferrara, P.; Apostolakis, J.; Caflisch, A., Evaluation of a fast implicit solvent model for molecular dynamics simulations. Proteins 2002, 46 (1), 24-33.

57. Lazaridis, T.; Karplus, M., Effective energy function for proteins in solution. Proteins 1999, 35 (2), 133-52.

58. Feig, M.; Karanicolas, J.; Brooks, C. L., 3rd, MMTSB Tool Set: enhanced sampling and multiscale modeling methods for applications in structural biology. $J \mathrm{Mol}$ Graph Model 2004, 22 (5), 377-95.

59. Khandogin, J.; Brooks, C. L., 3rd, Linking folding with aggregation in Alzheimer's beta-amyloid peptides. Proc Natl Acad Sci U S A 2007, 104 (43), 16880-5. 
60. Antzutkin, O. N.; Balbach, J. J.; Leapman, R. D.; Rizzo, N. W.; Reed, J.; Tycko, R., Multiple quantum solid-state NMR indicates a parallel, not antiparallel, organization of beta-sheets in Alzheimer's beta-amyloid fibrils. Proc Natl Acad Sci US A 2000, 97 (24), 13045-50.

61. Benzinger, T. L.; Gregory, D. M.; Burkoth, T. S.; Miller-Auer, H.; Lynn, D. G.; Botto, R. E.; Meredith, S. C., Two-dimensional structure of beta-amyloid(10-35) fibrils. Biochemistry 2000, 39 (12), 3491-9.

62. Petkova, A. T.; Ishii, Y.; Balbach, J. J.; Antzutkin, O. N.; Leapman, R. D.; Delaglio, F.; Tycko, R., A structural model for Alzheimer's beta-amyloid fibrils based on experimental constraints from solid state NMR. Proc Natl Acad Sci U S A 2002, 99 (26), 16742-7.

63. Murphy, R. M., Peptide aggregation in neurodegenerative disease. Annu Rev Biomed Eng 2002, 4, 155-74.

64. Inouye, H.; Kirschner, D. A., A beta fibrillogenesis: kinetic parameters for fibril formation from congo red binding. J Struct Biol 2000, 130 (2-3), 123-9.

65. Hortschansky, P.; Schroeckh, V.; Christopeit, T.; Zandomeneghi, G.; Fandrich, M., The aggregation kinetics of Alzheimer's beta-amyloid peptide is controlled by stochastic nucleation. Protein Sci 2005, 14 (7), 1753-9.

66. Bitan, G.; Vollers, S. S.; Teplow, D. B., Elucidation of primary structure elements controlling early amyloid beta-protein oligomerization. J Biol Chem 2003, 278 (37), 34882-9.

67. Hortschansky, P.; Christopeit, T.; Schroeckh, V.; Fandrich, M., Thermodynamic analysis of the aggregation propensity of oxidized Alzheimer's beta-amyloid variants. Protein Sci 2005, 14 (11), 2915-8.

68. Auer, S.; Dobson, C. M.; Vendruscolo, M.; Maritan, A., Self-templated nucleation in peptide and protein aggregation. Phys Rev Lett 2008, 101 (25), 258101.

69. Gursky, O.; Aleshkov, S., Temperature-dependent beta-sheet formation in betaamyloid Abeta(1-40) peptide in water: uncoupling beta-structure folding from aggregation. Biochim Biophys Acta 2000, 1476 (1), 93-102.

70. Lee, J. P.; Stimson, E. R.; Ghilardi, J. R.; Mantyh, P. W.; Lu, Y. A.; Felix, A. M.; Llanos, W.; Behbin, A.; Cummings, M.; Van Criekinge, M.; et al., 1H NMR of A beta amyloid peptide congeners in water solution. Conformational changes correlate with plaque competence. Biochemistry 1995, 34 (15), 5191-200. 
71. Kheterpal, I.; Williams, A.; Murphy, C.; Bledsoe, B.; Wetzel, R., Structural features of the Abeta amyloid fibril elucidated by limited proteolysis. Biochemistry 2001, 40 (39), 11757-67.

72. Takano, K., Amyloid beta conformation in aqueous environment. Curr Alzheimer Res 2008, 5 (6), 540-7.

73. Baumketner, A.; Bernstein, S. L.; Wyttenbach, T.; Bitan, G.; Teplow, D. B.; Bowers, M. T.; Shea, J. E., Amyloid beta-protein monomer structure: a computational and experimental study. Protein Sci 2006, 15 (3), 420-8.

74. Garai, K.; Sahoo, B.; Sengupta, P.; Maiti, S., Quasihomogeneous nucleation of amyloid beta yields numerical bounds for the critical radius, the surface tension, and the free energy barrier for nucleus formation. J Chem Phys 2008, 128 (4), 045102.

75. Chapagain, P. P.; van Kessel, J. S.; Karns, J. S.; Wolfgang, D. R.; Hovingh, E.; Nelen, K. A.; Schukken, Y. H.; Grohn, Y. T., A mathematical model of the dynamics of Salmonella Cerro infection in a US dairy herd. Epidemiol Infect 2008, 136 (2), 263-72.

76. Lloyd, A. L., Realistic distributions of infectious periods in epidemic models: changing patterns of persistence and dynamics. Theor Popul Biol 2001, 60 (1), 59-71.

77. Keeling, M. J.; Rohani, P., Modeling infectious diseases in humans and animals. Princeton University Press: Princeton, 2008; p xi, 366 p.

78. Lansbury, P. T., A Reductionist View of Alzheimer's Disease. Accounts of Chemical Research 1996, 29 (7), 317-321.

79. Perczel, A.; Park, K.; Fasman, G. D., Analysis of the circular dichroism spectrum of proteins using the convex constraint algorithm: a practical guide. Anal Biochem 1992, 203 (1), 83-93.

80. Sreerama, N.; Venyaminov, S. Y.; Woody, R. W., Estimation of protein secondary structure from circular dichroism spectra: inclusion of denatured proteins with native proteins in the analysis. Anal Biochem 2000, 287 (2), 243-51.

81. Sengupta, P.; Garai, K.; Sahoo, B.; Shi, Y.; Callaway, D. J.; Maiti, S., The amyloid beta peptide (Abeta(1-40)) is thermodynamically soluble at physiological concentrations. Biochemistry 2003, 42 (35), 10506-13.

82. Williams, A. D.; Portelius, E.; Kheterpal, I.; Guo, J. T.; Cook, K. D.; Xu, Y.; Wetzel, R., Mapping abeta amyloid fibril secondary structure using scanning proline mutagenesis. J Mol Biol 2004, 335 (3), 833-42. 
83. Zagorski, M. G.; Yang, J.; Shao, H.; Ma, K.; Zeng, H.; Hong, A., Methodological and chemical factors affecting amyloid beta peptide amyloidogenicity. Methods Enzymol 1999, 309, 189-204.

84. Feig, M., Modeling solvent environments : applications to simulations of biomolecules. Wiley-VCH: Weinheim, 2010; p xviii, 316 p.

85. Buck, M., Trifluoroethanol and colleagues: cosolvents come of age. Recent studies with peptides and proteins. Q Rev Biophys 1998, 31 (3), 297-355.

86. Roccatano, D.; Colombo, G.; Fioroni, M.; Mark, A. E., Mechanism by which 2,2,2-trifluoroethanol/water mixtures stabilize secondary-structure formation in peptides: a molecular dynamics study. Proc Natl Acad Sci U S A 2002, 99 (19), 1217984.

87. Santiveri, C. M.; Pantoja-Uceda, D.; Rico, M.; Jimenez, M. A., Beta-hairpin formation in aqueous solution and in the presence of trifluoroethanol: a (1)H and (13)C nuclear magnetic resonance conformational study of designed peptides. Biopolymers 2005, 79 (3), 150-62.

88. Karshikoff, A., Non-covalent interactions in proteins. Imperial College Press : World Scientific: London ; Hackensack, 2006; p xii, 333 p.

89. Anderson, C. O.; Spiegelberg, S.; Prausnitz, J. M.; Blanch, H. W., Effect of secondary structure on the interactions of peptide T4 LYS (11-36) in mixtures of aqueous sodium chloride and 2,2,2,-Trifluoroethanol. 2001.

90. Chu, H. L.; Lin, S. Y., Temperature-induced conformational changes in amyloid beta(1-40) peptide investigated by simultaneous FT-IR microspectroscopy with thermal system. Biophys Chem 2001, 89 (2-3), 173-80.

91. Soto, C.; Castano, E. M.; Frangione, B.; Inestrosa, N. C., The alpha-helical to beta-strand transition in the amino-terminal fragment of the amyloid beta-peptide modulates amyloid formation. J Biol Chem 1995, 270 (7), 3063-7.

92. Lazo, N. D.; Grant, M. A.; Condron, M. C.; Rigby, A. C.; Teplow, D. B., On the nucleation of amyloid beta-protein monomer folding. Protein Sci 2005, 14 (6), 1581 96.

93. Tomaselli, S.; Esposito, V.; Vangone, P.; van Nuland, N. A.; Bonvin, A. M.; Guerrini, R.; Tancredi, T.; Temussi, P. A.; Picone, D., The alpha-to-beta conformational transition of Alzheimer's Abeta-(1-42) peptide in aqueous media is reversible: a step by step conformational analysis suggests the location of beta conformation seeding. Chembiochem 2006, 7 (2), 257-67. 
94. Velez-Vega, C.; Escobedo, F. A., Characterizing the structural behavior of selected Abeta-42 monomers with different solubilities. J Phys Chem B 2011, 115 (17), 4900-10.

95. Szabo, Z.; Klement, E.; Jost, K.; Zarandi, M.; Soos, K.; Penke, B., An FT-IR study of the beta-amyloid conformation: standardization of aggregation grade. Biochem Biophys Res Commun 1999, 265 (2), 297-300.

96. Ma, B.; Nussinov, R., Polymorphic C-terminal beta-sheet interactions determine the formation of fibril or amyloid beta-derived diffusible ligand-like globulomer for the Alzheimer Abeta42 dodecamer. J Biol Chem 2010, 285 (47), 37102-10.

97. Sabate, R.; Estelrich, J., Evidence of the existence of micelles in the fibrillogenesis of beta-amyloid peptide. J Phys Chem B 2005, 109 (21), 11027-32.

98. Perutz, M. F.; Windle, A. H., Cause of neural death in neurodegenerative diseases attributable to expansion of glutamine repeats. Nature 2001, 412 (6843), 143-4.

99. Pellarin, R.; Caflisch, A., Interpreting the aggregation kinetics of amyloid peptides. J Mol Biol 2006, 360 (4), 882-92.

100. Wang, S. S. S.; Chen, Y.-T.; Chen, P.-H.; Liu, K.-N., A kinetic study on the aggregation behavior of $\beta$-amyloid peptides in different initial solvent environments. Biochemical Engineering Journal 2006, 29 (1-2), 129-138.

101. Kusumoto, Y.; Lomakin, A.; Teplow, D. B.; Benedek, G. B., Temperature dependence of amyloid beta-protein fibrillization. Proc Natl Acad Sci U S A 1998, 95 (21), 12277-82.

102. Tomski, S. J.; Murphy, R. M., Kinetics of aggregation of synthetic beta-amyloid peptide. Arch Biochem Biophys 1992, 294 (2), 630-8.

103. Yong, W.; Lomakin, A.; Kirkitadze, M. D.; Teplow, D. B.; Chen, S. H.; Benedek, G. B., Structure determination of micelle-like intermediates in amyloid beta -protein fibril assembly by using small angle neutron scattering. Proc Natl Acad Sci U S A 2002, 99 (1), 150-4.

104. Lee, C. F., Self-assembly of protein amyloids: a competition between amorphous and ordered aggregation. Phys Rev E Stat Nonlin Soft Matter Phys 2009, 80 (3 Pt 1), 031922 .

105. Sachse, C.; Xu, C.; Wieligmann, K.; Diekmann, S.; Grigorieff, N.; Fandrich, M., Quaternary structure of a mature amyloid fibril from Alzheimer's Abeta(1-40) peptide. J Mol Biol 2006, 362 (2), 347-54. 
106. Derreumaux, P., Alzheimer's disease : insights into low molecular weight and cytotoxic aggregates from in vitro and computer experiments : molecular basis of amyloid-beta protein aggregation and fibril formation. Imperial College Press ; Distributed by World Scientific Publishing Co. Pte. Ltd.: London Singapore, 2013; p xix, 444 pages.

107. Rathore, N.; Chopra, M.; de Pablo, J. J., Optimal allocation of replicas in parallel tempering simulations. J Chem Phys 2005, 122 (2), 024111.

108. Kone, A.; Kofke, D. A., Selection of temperature intervals for parallel-tempering simulations. J Chem Phys 2005, 122 (20), 206101.

109. Isobe, T., Amyloid and amyloidosis. Plenum Press: New York, 1988; p xx, 885 p.

110. Tycko, R., Molecular structure of amyloid fibrils: insights from solid-state NMR. QRev Biophys 2006, 39 (1), 1-55.

111. Li, D. W.; Mohanty, S.; Irback, A.; Huo, S., Formation and growth of oligomers: a Monte Carlo study of an amyloid tau fragment. PLoS Comput Biol 2008, 4 (12), e1000238.

112. Yu, L.; Edalji, R.; Harlan, J. E.; Holzman, T. F.; Lopez, A. P.; Labkovsky, B.; Hillen, H.; Barghorn, S.; Ebert, U.; Richardson, P. L.; Miesbauer, L.; Solomon, L.; Bartley, D.; Walter, K.; Johnson, R. W.; Hajduk, P. J.; Olejniczak, E. T., Structural characterization of a soluble amyloid beta-peptide oligomer. Biochemistry 2009, 48 (9), 1870-7.

113. Demus, D., Physical properties of liquid crystals. Wiley-VCH: Weinheim ; New York, 1999; p xix, 503 p.

114. Knowles, T. P.; De Simone, A.; Fitzpatrick, A. W.; Baldwin, A.; Meehan, S.; Rajah, L.; Vendruscolo, M.; Welland, M. E.; Dobson, C. M.; Terentjev, E. M., Twisting transition between crystalline and fibrillar phases of aggregated peptides. Phys Rev Lett 2012, 109 (15), 158101.

115. Chapagain, P. P.; Gerstman, B. S., Removal of kinetic traps and enhanced protein folding by strategic substitution of amino acids in a model alpha-helical hairpin peptide. Biopolymers 2006, 81 (3), 167-78.

116. Larini, L.; Shea, J. E., Role of beta-hairpin formation in aggregation: the selfassembly of the amyloid-beta(25-35) peptide. Biophys $J$ 2012, 103 (3), 576-86.

117. Gill, A. C., beta-hairpin-mediated formation of structurally distinct multimers of neurotoxic prion peptides. PLoS One 2014, 9 (1), e87354. 
118. Odian, G. G., Principles of polymerization. 4th ed.; Wiley: Hoboken, N.J., 2004; p xxiv, 812 p.

119. Hosia, W.; Bark, N.; Liepinsh, E.; Tjernberg, A.; Persson, B.; Hallen, D.; Thyberg, J.; Johansson, J.; Tjernberg, L., Folding into a beta-hairpin can prevent amyloid fibril formation. Biochemistry 2004, 43 (16), 4655-61.

120. Hoyer, W.; Hard, T., Interaction of Alzheimer's A beta peptide with an engineered binding protein--thermodynamics and kinetics of coupled folding-binding. $J \mathrm{Mol} \mathrm{Biol}$ 2008, 378 (2), 398-411.

121. Nord, K.; Gunneriusson, E.; Ringdahl, J.; Stahl, S.; Uhlen, M.; Nygren, P. A., Binding proteins selected from combinatorial libraries of an alpha-helical bacterial receptor domain. Nat Biotechnol 1997, 15 (8), 772-7.

122. Hoyer, W.; Gronwall, C.; Jonsson, A.; Stahl, S.; Hard, T., Stabilization of a betahairpin in monomeric Alzheimer's amyloid-beta peptide inhibits amyloid formation. Proc Natl Acad Sci U S A 2008, 105 (13), 5099-104. 
VITA

TIMOTHY M. STECKMANN

2009-2015 Teaching Assistant

Florida International University

Miami, Florida, USA

2006-2007 Teaching English as a Second Language

English Friends School

Busan, South Korea

2004-2005 Teaching English as a Second Language

Ding Ding Dong English School

Pohang, South Korea

2001-2004 Master of Science in Physics

Oregon State University

Corvallis, Oregon, USA

1992-1999 Bachelor of Science in Physics

Oregon State University

Corvallis, Oregon, USA

\section{PUBLICATIONS AND PRESENTATIONS}

Steckmann, T.; Awan, Z.; Gerstman, B. S.; Chapagain, P. P., Kinetics of peptide secondary structure conversion during amyloid beta-protein fibrillogenesis. $J$ Theor Biol 2012, 301, 95-102.

American Physical Society March Meeting, Denver, Colorado March 2014 "Structural Transitions and Aggregation in Amyloidogenic Proteins" 